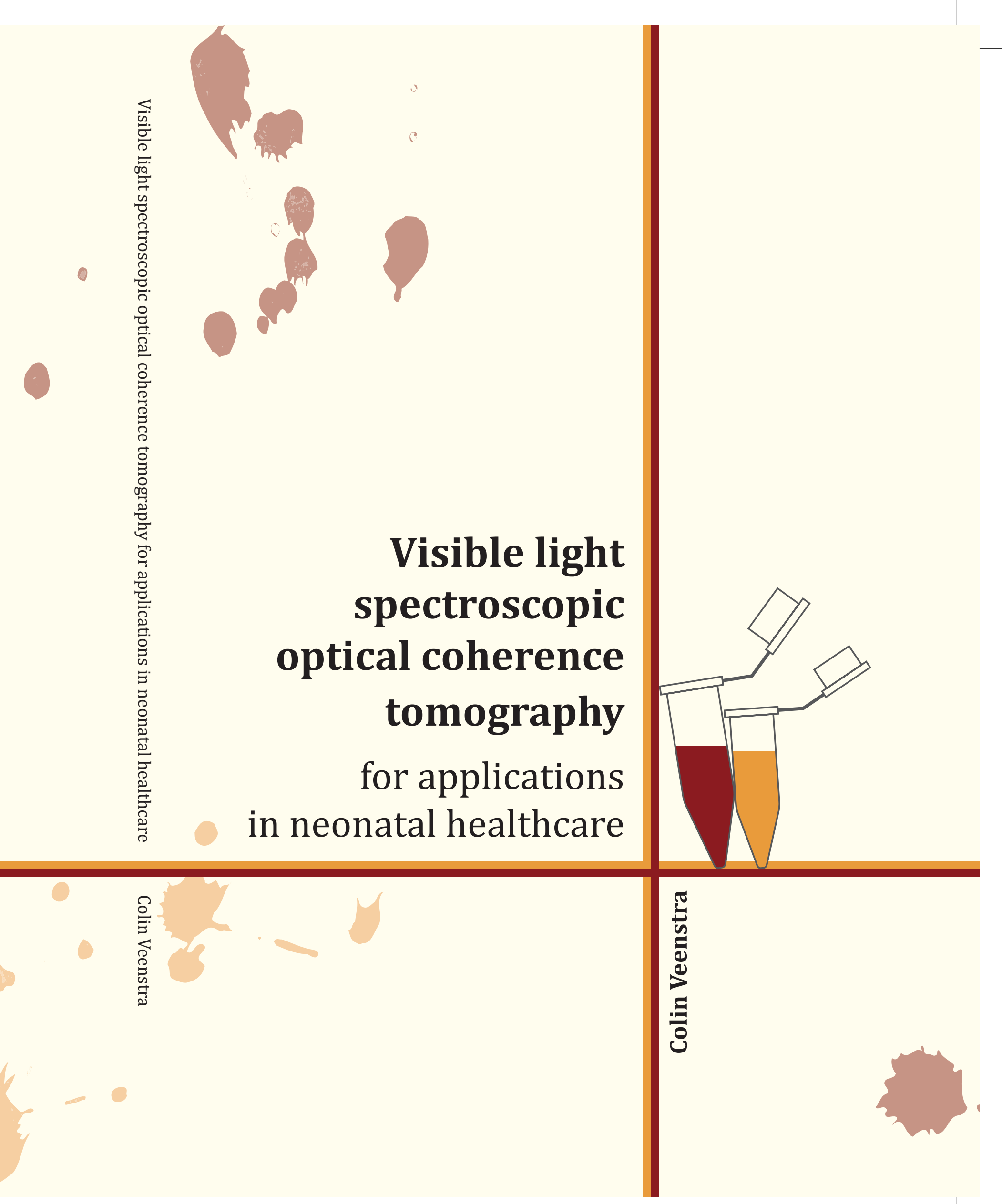




\section{VISIBLE LIGHT SPECTROSCOPIC OPTICAL COHERENCE TOMOGRAPHY FOR APPLICATIONS IN NEONATAL HEALTHCARE}

Colin Veenstra 


\title{
VISIBLE LIGHT SPECTROSCOPIC OPTICAL COHERENCE TOMOGRAPHY FOR APPLICATIONS \\ IN NEONATAL HEALTHCARE
}

\author{
PROEFSCHRIFT
}

\author{
ter verkrijging van \\ de graad van doctor aan de Universiteit Twente, \\ op gezag van de rector magnificus, \\ prof.dr. T.T.M. Palstra, \\ volgens besluit van het College voor Promoties \\ in het openbaar te verdedigen \\ op woensdag 10 juni 2020 om 12.45 uur
}

door

\section{Colin Veenstra}

geboren op 27 september 1991

te Almelo, Nederland 
Dit proefschrift is goedgekeurd door:

de promotor:

prof.dr.ir. W. Steenbergen

de co-promotor:

dr.ir. N. Bosschaart

Het werk in dit proefschrift is financieel ondersteund door The Innovation Research Incentives Scheme of The Netherlands Organisation for Scientific Research (NWO), division Applied and Engineering Sciences (TTW), The Pioneers in Healthcare Innovation Fund (Universiteit Twente) en de Universiteit Twente.

Cover design: Sjoukje Schoustra

Printed by: Gildeprint

Lay-out: Colin Veenstra

ISBN: 978-90-365-4987-5

DOI: $10.3990 / 1.9789036549875$

(C) 2019 Colin Veenstra, The Netherlands. all rights reserved. No parts of this thesis may be reproduced, stored in a retrieval system or transmitted in any form or by any means without permission of the author. Alle rechten voorbehouden. Niets uit deze uitgave mag worden vermenigvuldigd, in enige vorm of op enige wijze, zonder voorafgaande schriftelijke toestemming van de auteur. 


\section{PROMOTIE COMMISSIE:}

$\begin{array}{lll}\text { Voorzitter/secretaris: } & \text { prof.dr. J.L. Herek } & \text { Universiteit Twente } \\ \text { Promotor: } & \text { prof.dr.ir. W. Steenbergen } & \text { Universiteit Twente } \\ \text { Co-promotor: } & \text { dr.ir. N. Bosschaart } & \text { Universiteit Twente } \\ \text { Leden: } & \text { prof.dr. S. Manohar } & \text { Universiteit Twente } \\ & \text { prof.dr.ir. I.M. Vellekoop } & \text { Universiteit Twente } \\ & \text { prof.dr. A.G.J.M. van Leeuwen } & \text { Amsterdam UMC } \\ & \text { dr.ir. D.J. Faber } & \text { Amsterdam UMC } \\ & \text { prof.dr. A. Amelink } & \text { VU Amsterdam }\end{array}$




\section{Contents}

Chapter 1: Introduction to the thesis $\quad 8$

1.1 Biomedical optics 9

1.2 Optical Coherence Tomography (sOCT) 11

1.3 Optical properties 14

1.4 Contents of this thesis $\quad 16$

$\begin{array}{ll}\text { Bibliography } & 17\end{array}$

Chapter 2: Spatially confined quantification of bilirubin concentrations by spectroscopic visible-light optical coherence tomography

2.1 Introduction $\quad 22$

2.2 Methods $\quad 23$

2.3 Results $\quad 29$

2.4 Discussion and conclusion $\quad 31$

Bibliography $\quad 33$

Chapter 3: Quantification of total haemoglobin concentrations in human whole blood by spectroscopic visible-light optical coherence tomography

3.1 Introduction $\quad 36$

3.2 Results $\quad 37$

3.3 Discussion $\quad 39$

3.4 Conclusion $\quad 42$

3.5 Methods $\quad 42$

Bibliography $\quad 48$

Chapter 4: Optical properties of human milk 51

4.1 Introduction $\quad 52$

4.2 Materials and methods $\quad 54$

4.3 Results $\quad 62$

4.4 Discussion $\quad 66$

4.5 Conclusion $\quad 71$

Bibliography $\quad 71$ 
Chapter 5: The dependency of the optical scattering properties of human milk on casein content and common sample preparation methods

5.1 Introduction $\quad 78$

5.2 Materials and methods $\quad 80$

5.3 Results $\quad 86$

5.4 Discussion $\quad 90$

5.5 Conclusions $\quad 93$

Bibliography $\quad 93$

Chapter 6: Overall discussion, future perspectives and conclusions

6.1 Discussion $\quad 99$

6.2 Future perspectives $\quad 103$

6.3 Conclusions $\quad 104$

Bibliography $\quad 104$

List of symbols $\quad 106$

List of abbreviations $\quad 108$

Summary 109

Nederlandstalige samenvatting $\quad 112$

List of publications and presentations $\quad 115$

Dankwoord / Acknowledgements $\quad 117$

About the author $\quad 120$ 


\section{Chapter 1:}

\section{Introduction to the thesis}

Abstract: This chapter will introduce the thesis as a whole. We will discuss the opportunities and relevance of biomedical optics in general and spectroscopic optical coherence tomography in particular for neonatal healthcare. We briefly introduce and explain the optical properties which are used throughout the thesis. At the end of this chapter, an outline of the thesis is given. 


\subsection{Biomedical Optics}

\subsubsection{Optical imaging}

Biomedical optics is the field of research using the interaction between light and tissue. Individual scattering and absorption characteristics of various tissues allow for structural and functional imaging with high contrast. Ballistic optical imaging techniques such as confocal microscopy provide sub-micrometer resolution and a sub-millimeter penetration depth [1]. Infrared optical coherence tomography can measure up to several millimeters deep [2] with a resolution around $5 \mu \mathrm{m}$ [3], while using visible light optical coherence tomography improves the resolution to approximately $1 \mu \mathrm{m}$ [3]. Diffuse optical imaging allows for imaging with optical contrast for up to several centimeters with sub-millimeter resolution [4].

\subsubsection{Spectroscopy}

Since optical scatterers and especially absorbers exhibit characteristic wavelength dependent scattering and absorption features respectively, spectroscopic methods give rise to additional contrast between tissues. Furthermore, spectrally resolved detection - often combined with a model for light transport - allows for decoupling the individual contributions of scattering and chromophores (e.g. oxyhemoglobin, deoxyhemoglobin and bilirubin) to the attenuation of light within the probed volume [5]. Transmission spectroscopy allows for estimating chromophore concentrations in non-scattering media by measuring the attenuation of light through a sample with known thickness (i.e. known optical path length) as a function of wavelength $\lambda$. Nevertheless, conventional spectroscopic methods do not allow for controlling or measuring the optical path length in scattering media, and therefore require assumptions about the optical path length and sample structure to assign quantitative values to the attenuation of light. As a result, most conventional spectroscopic methods have limited accuracy when quantifying chromophore concentrations within a confined region of interest.

\subsubsection{Opportunities for neonatal healthcare}

\subsubsection{Blood analysis in newborns}

It is important to monitor hemoglobin and bilirubin concentration in neonates; Hemoglobin is the oxygen transporting protein which gives the blood its red 
color. Anemia - a shortage of hemoglobin - may lead to organ damage due to oxygen deficiency. Bilirubin is the yellow breakdown product of hemoglobin, which at elevated levels may cause brain damage [6]. Since both hemoglobin and bilirubin exhibit strong optical absorption in the visible wavelength range $[7,8]$, biomedical optics offers many opportunities for neonatal healthcare. A developing technique called spectroscopic optical coherence tomography (sOCT) has the potential of quantifying chromophore concentrations inside single blood vessels $[5,9]$ and might therefore prove to be a non-invasive alternative for quantifying the hemoglobin and bilirubin in the blood of newborns. Currently, the gold standard for estimating hemoglobin and bilirubin levels in blood is invasive blood sampling [10]. Although these invasive methods are accurate, invasive blood sampling in high-risk groups such as newborns may cause pain, stress and significant blood loss [11]. Non-invasive transcutaneous bilirubinometers exist, but lack the accuracy to completely replace invasive blood sampling [12]. Therefore an obvious need exists for accurate non-invasive alternatives.

\subsubsection{Human milk and lactation}

SOCT is not only sensitive to absorption, but also to scattering [13]. This opens up new opportunities for neonatal healthcare, for example in the research field of human lactation [14]. Breastfeeding comes with many advantages for both mother and child, possibly averting the death of 823,000 children and 20,000 mothers each year if all mothers worldwide would breastfeed their infants [15]. However, breastfeeding rates at the age of 1 year are less than $20 \%$ in many high-income countries. The most important reasons for mothers to stop breastfeeding are the perception of insufficient milk supply and pain due to breastfeeding problems [16]. Since SOCT is sensitive for both scattering and absorption, this technique can be utilized for measuring the - up to this point unknown interaction between human milk and light. Since fat and casein micelles are the most dominant scatterers in milk $[17,18]$, it is very interesting to explore the correlation between light scattering and the nutritional content of human milk. Developing a quick and easy to use home test for mothers to estimate the nutritional content of their milk might take away uncertainty about the energy intake of their child. 


\subsection{Optical Coherence Tomography (OCT)}

\subsubsection{Conventional OCT}

Optical coherence tomography [19] is an optical imaging technique based on low coherence interferometry. An illustration of a basic spectral domain (i.e. using a spectrometer) OCT setup is given in figure 1.1. A broadband light source emits the light towards a beam splitter, guiding a certain fraction of the light towards the sample and the remainder towards the reference arm. In the sample arm, the light is backscattered by the sample. In the reference arm, the light is reflected back by a mirror. The light is combined again at the beam splitter, after which it is guided towards a spectrometer. Due to interference between both arms, the detected light is modulated as a function of the optical path length difference $(\triangle \mathrm{OPL})$ between the sample and reference arm:

$$
I_{\text {det }}(k) \propto I_{S}(k)+I_{r}(k)+2 \sqrt{I_{S}(k) I_{r}(k)} \cos (k \Delta O P L)
$$

with $I_{d e t}, I_{s}$ and $I_{r}$ the intensities at the detector, in the sample arm and in the reference arm respectively and $k$ the angular wave number $(k=2 \pi / \lambda)$. Fourier transformation of $I_{\text {det }}(k)$ results in a $1 \mathrm{D}$-image (A-scan) - i.e. the backscattered light from the sample as a function of $\triangle O P L$ - meaning that the optical path length through the medium is both known and controllable. OCT allows for $2 \mathrm{D}$
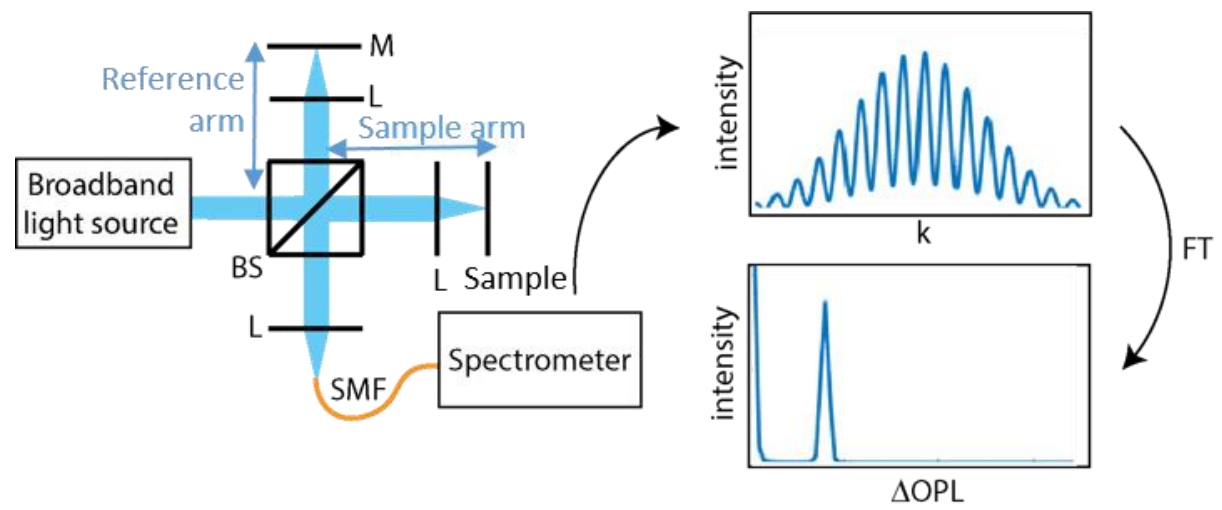

Figure 1.1: Illustration of a basic spectral domain OCT setup. Light reflected from both arms interferes at the spectrometer, resulting in a modulated signal. Fourier transformation of this signal yields the optical path length difference between both arms. BS: Beam splitter, L: Lens, M: Mirror, SMF: Single mode fiber, FT: Fourier transformation. 
and 3D imaging by scanning the sample laterally and concatenating the resulting individual A-scans.

Since OCT images are created from the light backscattered by the sample, contrast in these images is mainly based on scattering. The lateral resolution is determined by the used optics, while the axial resolution is given by the coherence length $L_{C}$ (assuming a Gaussian source spectrum):

$$
L_{C}=\frac{2 \ln 2}{\pi} \frac{\lambda_{\text {center }}^{2}}{n \lambda_{F W H M}}
$$

With $\lambda_{\text {center }}$ the center wavelength and $\lambda_{F W H M}$ the bandwidth (full-width-halfmaximum) of the detected spectrum and $n$ the refractive index of the sample.

In spectral domain OCT, the backscattered signal as a function of depth $(=\Delta \mathrm{OPL} / 2 \mathrm{n})$ not only depends on the optical properties of the sample, but is also affected by defocus and roll-off [9]. Defocus occurs since the focus lens in the sample arm can only focus at a single position inside the sample, resulting in a higher backscattered intensity at that position. The so-called point spread function (PSF) relates the radius of the beam at a distance / from focus position $I_{f}$ to the Rayleigh length $Z_{R}$ of the system:

$$
P S F\left(l_{f}-l\right)=\alpha\left(\left(\frac{l_{f}-l}{2 Z_{R}}\right)^{2}+1\right)^{-1}
$$

with scaling factor $\alpha$. Roll-off decreases the spectrometer sensitivity with depth and is caused by the finite size of the pixels inside the spectrometer. The magnitude of the decrease as a function of depth $d$ is given by [20]:

$$
R O(d)=\left(\frac{\sin \zeta}{\zeta}\right)^{2} \exp \left(-\frac{w^{2}}{2 \ln 2} \tau^{2}\right)
$$

where $R O$ is the relative sensitivity, $\tau=(\pi / 2)\left(d / d_{\max }\right)$ denotes the depth relative to the maximum depth $d_{\max }=\lambda^{2} /(4 \xi \lambda)$ where $\xi \lambda$ is the wavelength spacing between pixels and $w=\delta \lambda / \xi \lambda$ where $\delta \lambda$ is the spectral resolution of the spectrometer.

\subsubsection{Spectroscopic OCT (sOCT)}

SOCT is a functional extension of conventional OCT [21], which combines contrast between chromophores from conventional spectroscopy with quantification of the optical path length from OCT. Contrary to conventional 
a)
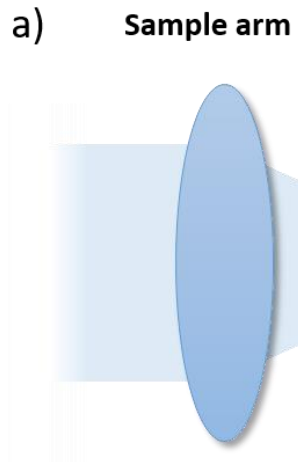

b)

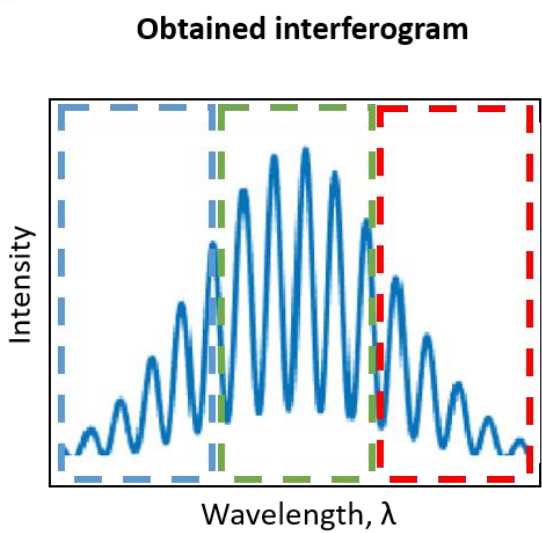

Tissue containing Oxyhemoglobin absorption

Blood vessels spectrum

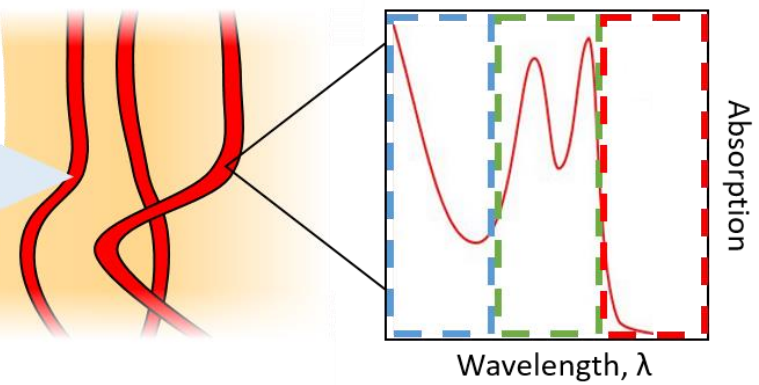

Individual backscattered intensities For every wavelength region
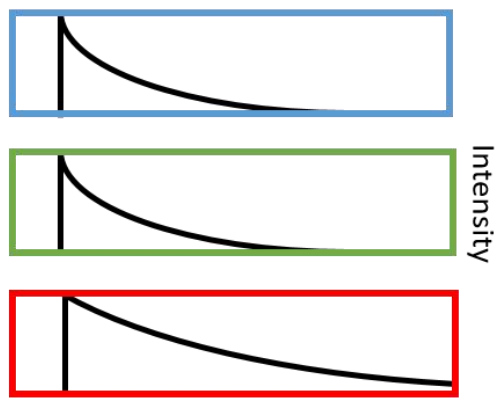

$\triangle \mathrm{OPL}$

Figure 1.2: Illustration of Short time Fourier transformation in SOCT within a single blood vessel. The blue, green and red boxes indicate the same wavelength regions throughout this figure. a) OCT allows for localized measurements of the backscattered intensity within a single blood vessel. Blood exhibits a wavelength dependent absorption - generally dominated by oxygenated hemoglobin which also contributes to the wavelength dependency of the backscattered signal. b) In short time Fourier transformation, the Fourier transformation is applied only within a confined wavelength window. By sliding the window, the backscattered intensity as a function of $\triangle O P L$ is obtained separately for every wavelength region resulting in spectral resolution at the cost of spatial resolution. For longer wavelengths (indicated by the red box), the backscattered intensity shows a slower decay with $\triangle O P L$ due to the lower absorption by blood in this wavelength region (assuming wavelength-independent scattering). 
spectroscopy, this allows for quantitative spectroscopy in a confined region of interest without crosstalk from surrounding tissue.

SOCT is performed by applying a short time Fourier transformation (instead of a regular Fourier transformation) on $I_{\text {det }}(k)$, resulting in the backscattered intensity as a function of both $\triangle O P L$ and wavelength (Figure 1.2) [21]. SOCT has a trade-off between spectral and spatial (axial) resolution $\Delta d$, which is controlled by the spectral width $\Delta \lambda$ of the window used during short time Fourier transformation:

$$
\Delta d=\frac{\lambda^{2}}{2 n \Delta \lambda}
$$

Both oxygenated and deoxygenated hemoglobin have a very high and characteristic absorption in the visible wavelength range [8], this results in visible-light SOCT not only being employed to create true color OCT images [22], but also for localized in-vivo estimation of the oxygen saturation in single blood vessels [23-27] and in-vivo quantification of hemoglobin concentrations [28].

\subsection{Optical Properties}

The interaction between a medium and light can be described by the medium's optical properties. For readability reasons, all variables with a dependency on wavelength are marked bold in this section. Quantification of the optical properties may reveal information about the structure and contents of the probed volume. Attenuation of light inside tissue takes place through absorption and scattering of light. Absorption can be described quantitatively by the absorption coefficient $\boldsymbol{\mu}_{a}$, quantifying the fraction of light absorbed with distance and thus has units of reciprocal distance $\left[\mathrm{m}^{-1}\right] . \boldsymbol{\mu}_{a}$ is, assuming spherically shaped absorbing particles, related to the details of the medium by:

$$
\mu_{\boldsymbol{a}}=\boldsymbol{Q}_{\boldsymbol{a}} \pi r^{2} N_{a}
$$

With absorption efficiency $\boldsymbol{Q}_{\boldsymbol{a}}$, absorber cross section $\pi r^{2}$ and number of absorbers per volume $N_{a}$. Note that $\boldsymbol{\mu}_{a}$ is directly related to chromophore concentration (e.g. hemoglobin, bilirubin) through $N_{a}$, meaning that quantification of $\boldsymbol{\mu}_{a}$ allows for quantitative estimation of chromophore concentrations.

Light loses directionality when it encounters variations in the (real part of the) refractive index, a process called scattering. Similar to absorption, the fraction 
of scattered light per unit of distance is described by scattering coefficient $\boldsymbol{\mu}_{\boldsymbol{s}}$. Assuming no dependent scattering, $\boldsymbol{\mu}_{s}$ is defined as:

$$
\mu_{s}=Q_{s} \pi r^{2} N_{s}
$$

With scattering efficiency $\boldsymbol{Q}_{\boldsymbol{s}}$, scatterer cross section $\pi r^{2}$ and number of scatterers per volume $N_{s}$. Absorption and scattering both contribute to the attenuation of ballistic light, therefore we define attenuation coefficient $\boldsymbol{\mu}_{t}$ as:

$$
\mu_{t} \equiv \mu_{a}+\mu_{s}
$$

Since $\boldsymbol{\mu}_{s}$ only quantifies the amount of scattering events per distance, it holds no information about the change in directionality - commonly known as scattering angle - per scattering event. The scattering phase function $P(\Theta)$ gives the angular distribution of scattered light intensity at scattering angle $\Theta$, relative to the incident beam. From $\boldsymbol{P}(\boldsymbol{\theta})$ we can define backscatter coefficient $\boldsymbol{\mu}_{b}$, which quantitatively describes the amount of backscattered $(\Theta=\pi)$ light per unit of distance:

$$
\mu_{\boldsymbol{b}}=\mu_{\boldsymbol{s}} \boldsymbol{P}(\boldsymbol{\theta}=\boldsymbol{\pi})
$$

In this work, we measured $\boldsymbol{\mu}_{b, N A}$, the light backscattered within the numerical aperture (NA) of the system:

$$
\mu_{\boldsymbol{b}, \boldsymbol{N} \boldsymbol{A}}=\mu_{\boldsymbol{s}} 2 \pi \int_{\theta=\pi-N A}^{\pi} \boldsymbol{P}(\boldsymbol{\theta}) \sin \theta d \theta
$$

Another important scattering property is the anisotropy $\boldsymbol{g}$, which is directly related to $P(\Theta)$ and is defined as the average cosine of the scattering angle [29]:

$$
\boldsymbol{g} \equiv<\cos \boldsymbol{\theta}>
$$

For a purely forward scattering medium $\boldsymbol{g}=1$, whereas $\boldsymbol{g}=-1$ for purely backward scattering and $\boldsymbol{g}=0$ for isotropic scattering. The diffusion of light throughout a scattering medium is dependent on both, the amount of scattering events and the scattering angle, which are both incorporated into the reduced scattering coefficient $\boldsymbol{\mu}_{s}^{\prime}$ :

$$
\mu_{s}^{\prime}=\mu_{s}(1-g)
$$

It is very intuitive to mention that after light passes a distance of $1 / \mu_{s}{ }^{\prime}$ through a medium, it has lost all of its initial directionality. 


\subsection{Contents of this thesis}

We investigate how the benefits of SOCT and related optical techniques can be exploited to improve the neonatal healthcare. Due to the very high optical attenuation in the visible wavelength range $\left(\mu_{t} \approx 100 \mathrm{~mm}^{-1}\right.$ [8]), quantification of the optical properties of human whole blood requires an SOCT system with superior sensitivity. In such a system, the backscattered signal as a function of depth is only affected by the optical properties of the sample. Hereto, we developed an SOCT system that is capable of measuring the attenuation of light with superior sensitivity and without the influence of defocus and sensitivity roll-off (i.e. the measured attenuation is only affected by the sample itself) by combining focus tracking with zero delay $(\triangle O P L=0)$ acquisition. By matching the focus and zero delay position for every depth position inside the sample, we ensure in-focus measurements and optimal spectrometer sensitivity at every depth inside the sample.

In this thesis, we show the potential of our sOCT system to quantify hemoglobin and bilirubin concentrations. Furthermore we measure and present the full set of optical properties of human milk to explore the possibilities of visible-light for estimating the nutritional content of human milk.

In chapter 2, we show the potential of our sOCT system by locally quantifying bilirubin concentrations in neonatal-skin-mimicking samples. Furthermore, we quantify the bilirubin concentration behind a scattering layer. This chapter also introduces our home-built SOCT system, data processing and attenuation model, all of which are used throughout this thesis.

In chapter 3, we use our SOCT system to quantify total hemoglobin concentrations in human whole blood. Hereto, we place a capillary in the focus of the sample arm through which a flow of human blood was established. Since hemoglobin is generally encapsulated by red blood cells, we include absorption flattening into our attenuation model.

In chapter 4, we measure the full set of optical properties of human milk by combining SOCT with spatially resolved diffuse reflectance spectroscopy (SR$D R S)$. Scientific research on human milk is underrepresented, while visible-light biomedical optics might offer a wealth of opportunities for estimating the nutritional content of milk and for researching lactation physiology. Since everything starts with understanding the interaction between milk and light, we 
map the complete set of optical properties of human milk $\left(\boldsymbol{\mu}_{a}, \boldsymbol{\mu}_{s,}, \boldsymbol{\mu}_{s}^{\prime}, \boldsymbol{\mu}_{b, N A}, \boldsymbol{g}\right)$ and investigate the dependence of these optical properties on fat concentration.

In chapter 5, we investigate the dependency of the scattering properties of human milk on the preparation procedure. Prior to analysis and/or measurements, human milk samples can be homogenized and prepared by multiple techniques. The most common preparation methods are gently inverting the sample by hand, vortexing and ultrasonic sonication. We also measure the scattering properties of human milk samples with the casein micelles disintegrated, from which - using the difference from regular samples - we estimate their contribution to the total scattering properties of the sample.

In chapter 6, we discuss the work in this thesis and future perspectives. We describe our contribution to the field of biomedical optics for neonatal care. Furthermore, we discuss the future perspectives and outlook for further improvements of SOCT for non-invasive blood analysis and lactation research.

\section{Bibliography}

1. J. B. Pawley, Handbook of Biological Confocal Microscopy (Springer, Boston, MA, 2006).

2. A. F. Fercher, "Optical coherence tomography - development, principles, applications," Z Med Phys 20(4), 251-276 (2010).

3. X. Shu, L. Beckmann, and H. F. Zhang, "Visible-light optical coherence tomography: a review," J Biomed Opt 22(12) (2017).

4. G. Ku, and L. H. V. Wang, "Deeply penetrating photoacoustic tomography in biological tissues enhanced with an optical contrast agent," Opt Lett 30(5), 507-509 (2005).

5. N. Bosschaart, D. J. Faber, T. G. van Leeuwen, and M. C. G. Aalders, "In vivo low-coherence spectroscopic measurements of local hemoglobin absorption spectra in human skin," J Biomed Opt 16(10) (2011).

6. T. W. R. Hansen, "Prevention of neurodevelopmental sequelae of jaundice in the newborn," Dev Med Child Neurol 53, $24-28$ (2011).

7. S. Prahl, "Bilirubin," (2017).

8. N. Bosschaart, G. J. Edelman, M. C. G. Aalders, T. G. van Leeuwen, and D. J. Faber, "A literature review and novel theoretical approach on the optical properties of whole blood," Laser Med Sci 29(2), 453-479 (2014). 
9. N. Bosschaart, M. C. G. Aalders, T. G. van Leeuwen, and D. J. Faber, "Spectral domain detection in low-coherence spectroscopy," Biomed Opt Express 3(9), 2263-2272 (2012).

10. World Health Organization, "Haemoglobin concentrations for the diagnosis of anaemia and assessment of severity," (2011). at https://www.who.int/vmnis/indicators/haemoglobin/en/

11. G. L. Crighton, H. V. New, H. G. Liley, and S. J. Stanworth, "Patient blood management, what does this actually mean for neonates and infants?," Transfusion Med 28(2), 117-131 (2018).

12. M. D. van Erk, A. J. Dam-Vervloet, F. de Boer, M. F. Boomsma, H. van Straaten, and N. Bosschaart, "How skin anatomy influences transcutaneous bilirubin determinations: an in vitro evaluation," Pediatr. Res. 86, 471-477 (2019).

13. N. Bosschaart, D. J. Faber, T. G. van Leeuwen, and M. C. G. Aalders, "Measurements of wavelength dependent scattering and backscattering coefficients by low-coherence spectroscopy," J Biomed Opt 16(3) (2011).

14. M. C. Neville, S. M. Anderson, J. L. McManaman, T. M. Badger, M. Bunik, N. Contractor, T. Crume, D. Dabelea, S. M. Donovan, N. Forman, D. N. Frank, J. E. Friedman, J. B. German, A. Goldman, D. Hadsell, M. Hambidge, K. Hinde, N. D. Horseman, R. C. Hovey, E. Janoff, N. F. Krebs, C. B. Lebrilla, D. G. Lemay, P. S. MacLean, P. Meier, A. L. Morrow, J. Neu, L. A. Nommsen-Rivers, D. J. Raiten, M. Rijnkels, V. Seewaldt, B. D. Shur, J. VanHouten, and P. Williamson, "Lactation and Neonatal Nutrition: Defining and Refining the Critical Questions," J Mammary Gland Biol 17(2), 167-188 (2012).

15. C. G. Victora, R. Bahl, A. J. D. Barros, G. V. A. Franca, S. Horton, J. Krasevec, S. Murch, M. J. Sankar, N. Walker, N. C. Rollins, and L. B. S. Grp, "Breastfeeding in the 21st century: epidemiology, mechanisms, and lifelong effect," Lancet 387(10017), 475-490 (2016).

16. R. Li, S. B. Fein, J. Chen, and L. M. Grummer-Strawn, "Why mothers stop breastfeeding: mothers' self-reported reasons for stopping during the first year," Pediatrics 122 Suppl 2, S69-76 (2008).

17. S. Stocker, F. Foschum, P. Krauter, F. Bergmann, A. Hohmann, C. S. Happ, and A. Kienle, "Broadband Optical Properties of Milk," Appl Spectrosc 71(5), 951-962 (2017).

18. E. Bijl, R. de Vries, H. van Valenberg, T. Huppertz, and T. Van Hooijdonk, "Factors influencing casein micelle size in milk of individual 
cows: Genetic variants and glycosylation of kappa-casein," Int Dairy J 34(1), 135-141 (2014).

19. D. Huang, E. A. Swanson, C. P. Lin, J. S. Schuman, W. G. Stinson, W. Chang, M. R. Hee, T. Flotte, K. Gregory, C. A. Puliafito, and J. G.

Fujimoto, "Optical Coherence Tomography," Science 254(5035), 11781181 (1991).

20. S. H. Yun, G. J. Tearney, B. E. Bouma, B. H. Park, and J. F. de Boer, "High-speed spectral-domain optical coherence tomography at $1.3 \mathrm{mu}$ m wavelength," Opt Express 11(26), 3598-3604 (2003).

21. H. S. Nam, and H. Yoo, "Spectroscopic optical coherence tomography: A review of concepts and biomedical applications," Appl Spectrosc Rev 53(2-4), 91-111 (2018).

22. F. E. Robles, C. Wilson, G. Grant, and A. Wax, "Molecular imaging truecolour spectroscopic optical coherence tomography," Nat Photonics 5(12), 744-747 (2011).

23. S. H. Pi, A. Camino, W. Cepurna, X. Wei, M. Zhang, D. Huang, J. Morrison, and Y. L. Jia, "Automated spectroscopic retinal oximetry with visible-light optical coherence tomography," Biomed Opt Express 9(5), 2056-2067 (2018).

24. J. Yi, W. Z. Liu, S. Y. Chen, V. Backman, N. Sheibani, C. M. Sorenson, A. A. Fawzi, R. A. Linsenmeier, and H. F. Zhang, "Visible light optical coherence tomography measures retinal oxygen metabolic response to systemic oxygenation," Light-Sci Appl 4 (2015).

25. S. Y. Chen, J. Yi, and H. F. Zhang, "Measuring oxygen saturation in retinal and choroidal circulations in rats using visible light optical coherence tomography angiography," Biomed Opt Express 6(8), 28402853 (2015).

26. J. Yi, S. Y. Chen, V. Backman, and H. F. Zhang, "In vivo functional microangiography by visible-light optical coherence tomography," Biomed Opt Express 5(10), 3603-3612 (2014).

27. J. Yi, and X. Li, "Estimation of oxygen saturation from erythrocytes by high-resolution spectroscopic optical coherence tomography," Opt Lett 35(12), 2094-2096 (2010).

28. S. P. Chong, C. W. Merkle, C. Leahy, H. Radhakrishnan, and V. J. Srinivasan, "Quantitative microvascular hemoglobin mapping using visible light spectroscopic Optical Coherence Tomography," Biomed Opt Express 6(4), 1429-1450 (2015). 
29. B. C. Wilson, and S. L. Jacques, "Optical Reflectance and Transmittance of Tissues - Principles and Applications," leee J Quantum Elect 26(12), 2186-2199 (1990). 


\title{
Chapter 2:
}

\section{Spatially confined quantification of bilirubin concentrations by spectroscopic visible-light optical coherence tomography}

\begin{abstract}
Spatially confined measurements of bilirubin in tissue can be of great value for noninvasive bilirubin estimations during neonatal jaundice, as well as our understanding of the physiology behind bilirubin extravasation. This work shows the potential of spectroscopic visible-light optical coherence tomography (sOCT) for this purpose. At the bilirubin absorption peak around $460 \mathrm{~nm}$, sOCT suffers from a strong signal decay with depth, which we overcome by optimizing our system sensitivity through a combination of zero-delay acquisition and focus tracking. In a phantom study, we demonstrate the quantification of bilirubin concentrations between 0-650 $\mu \mathrm{M}$ with only $10 \%$ difference to the expected value, thereby covering the entire clinical pathophysiological range.
\end{abstract}

This chapter is published as: Colin Veenstra, Wilma Petersen, Ivo M. Vellekoop, Wiendelt Steenbergen, and Nienke Bosschaart, "Spatially confined quantification of bilirubin concentrations by spectroscopic visible-light optical coherence tomography," Biomed. Opt. Express 9(8), 3581-3589 (2018) 


\section{$2.1 \quad$ Introduction}

Bilirubin is the yellow, toxic breakdown product of hemoglobin, which is excreted by the body in bile and urine. Elevated levels of bilirubin lead to jaundice: a yellow discoloration of the eyes and skin. In case of severe jaundice (hyperbilirubinemia), bilirubin can accumulate in the brain and induce kernicterus, which results in irreversible brain damage [1]. Therefore, it is of vital importance to monitor bilirubin levels in high-risk population groups, such as newborns. Unfortunately, current clinically applied methods for bilirubin monitoring are either invasive (blood sampling), or are not accurate enough to fully replace invasive blood sampling (transcutaneous bilirubinometry by diffuse reflectance spectroscopy) $[2,3]$.

Here, we investigate the use of visible-light spectroscopic optical coherence tomography (SOCT) for noninvasive bilirubin determinations. The unique advantage of SOCT is that it allows for both quantitative and spatially confined measurements of bilirubin concentrations. This potentially enables the noninvasive determination of bilirubin concentrations within blood vessels, facilitating a direct comparison with invasive blood sampling without any cross talk from surrounding tissue. Since the existing transcutaneous bilirubinometers are unable to spatially resolve detected photons, cross talk from surrounding tissue is their main accuracy limiting factor [2]. Another important potential application of SOCT is the noninvasive study of local bilirubin extravasation into skin and brain tissue. As such a study is currently impossible, this may lead to a more fundamental understanding on the development of pathologies like kernicterus [2] and processes like the cephalocaudal progression of jaundice [4].

Multiple studies have shown the feasibility of SOCT and the closely related technology low-coherence spectroscopy (LCS) for the ex vivo $[5,6]$ and in vivo quantification of absorber concentrations in the visible wavelength range, including preclinical applications for highly localized tissue oximetry [7-13]. Using SOCT for bilirubin quantification in tissue introduces several sensitivityrelated challenges, as bilirubin absorbs around the relatively short wavelengths of $440 \mathrm{~nm}$ (free bilirubin) and $470 \mathrm{~nm}$ (albumin-bound bilirubin). This wavelength region is not only associated with impaired penetration depth into tissue, but also comes with a sharper roll-off of the sensitivity with depth due to the finite pixel size of the detecting spectrograph [14]. Hence, bilirubin determinations require an SOCT system with superior sensitivity, which we 
realize here by combining spectral domain SOCT with focus tracking and zerodelay acquisition throughout the entire axial scanning range. Besides the optimization of system sensitivity, this also ensures that the measured attenuation is only affected by the sample itself, resulting in quantitative measurements of the optical properties of the sample without requiring any calibration procedure.

In this work, we validate our SOCT system in the wavelength range of 440-600 $\mathrm{nm}$ for quantitative bilirubin determinations in samples mimicking neonatal skin. We show that our SOCT method is able to estimate bilirubin concentrations up to $650 \mu \mathrm{M}$ with an accuracy of $10 \%$ and a standard deviation in the order of $50 \mu \mathrm{M}$. The investigated bilirubin range covers the entire clinical pathophysiological range $(50-500 \mu \mathrm{M})[2,3]$. To demonstrate the spatially confined aspect of the technique, we also measured bilirubin concentrations behind an epidermis mimicking scattering layer with an accuracy of $10 \%$.

\section{$2.2 \quad$ Methods}

\subsubsection{Setup}

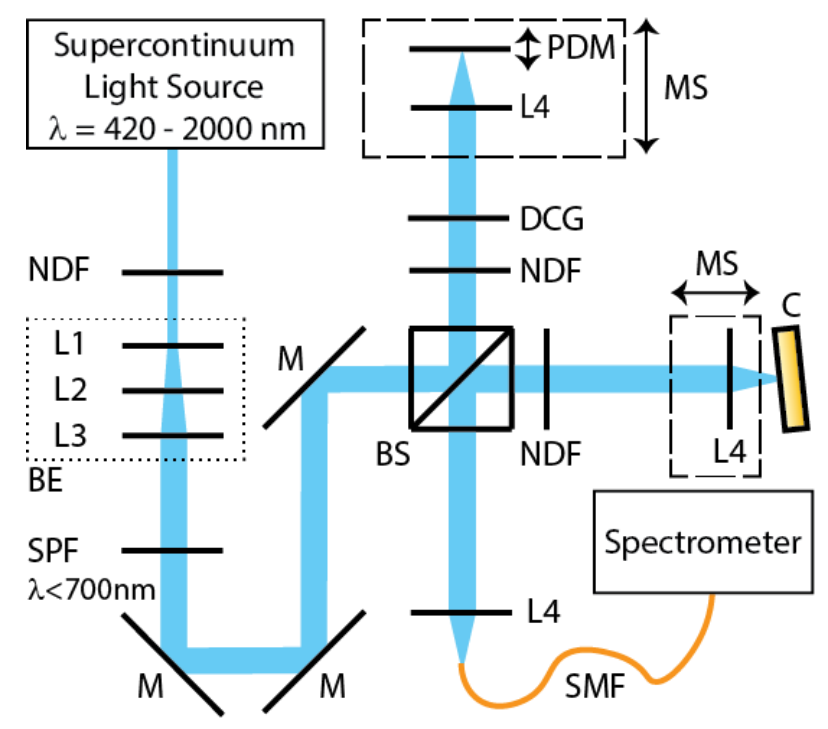

Figure 2.1: Schematic overview of the sOCT setup. NDF: neutral density filters, L\#: lens \#, BE: beam expander, SPF: short pass filter, M: mirror, BS: beam splitter, $D C G$ : dispersion compensation glass, MS: motorized stage, PDM: piezo driven mirror, C: cuvette with sample, SMF: single mode fiber. 
Our sOCT setup (Figure 2.1) is based on an open air Michelson interferometer, with a supercontinuum light source (SuperK EXTREME EXB-6, NKT Photonics, Denmark). Two neutral density filters (ND05A, Thorlabs, USA) attenuate the light (Total optical density = 1). Three lenses (LD2746-A, LD2060-A, LB1471-A, Thorlabs, USA) expand and collimate the beam, resulting in a beam with a diameter of $3 \mathrm{~mm}$ (full width at half maximum). A short pass filter (FESH0700, Thorlabs, USA) filters out all wavelengths longer than $700 \mathrm{~nm}$. Protected silver mirrors (PF10-03-P01, Thorlabs, USA) guide the beam into the desired direction. A 10:90 beam splitter (BS028, Thorlabs, USA) guides $10 \%$ of the light towards the sample arm and $90 \%$ towards the reference arm. In both, the sample arm (NDC-50C-2M-A, Thorlabs, USA) and the reference arm (NDC-50C-4M, Thorlabs, USA), the light intensity can be controlled by variable neutral density filters. Achromatic lenses (AC127-025-A, Thorlabs, USA) with a focal length of $25 \mathrm{~mm}$ focus the light on the sample contained by a glass cuvette, and a piezo-driven oscillating reference mirror. A motorized linear stage (T-LS13M, Zaber, USA) controls the reference arm length by joint translation of the reference mirror and its focusing lens. An identical stage facilitates focus tracking by translation of the focusing lens in the sample arm (see section 2.2.2.3). The back scattered light from both arms is guided by a single mode fiber (S405-XP, Thorlabs, USA) to a home-built spectrometer, where it is dispersed by a grating on a line scan camera (Sprint spL4096-140km, Basler, Germany) with a spectral resolution $\delta \lambda$ $=0.1 \mathrm{~nm}$ over a range of $440-600 \mathrm{~nm}$. This results in a theoretical axial resolution of $1.4 \mu \mathrm{m}$ in air.

\subsubsection{Data acquisition and processing}

A schematic overview of data acquisition and processing is shown in Figure 2.2. The optical path length difference between the sample and the reference arm $\triangle O P L=2 n\left(x_{s}-x_{r}\right)$, with $n$ the refractive index of the sample, and $x_{s}$ and $x_{r}$ the sample arm length and reference arm length respectively, induces a modulation on the detected intensity $I_{\text {det }}$ :

$$
I_{\mathrm{det}}(\lambda, t) \propto I_{s}(\lambda)+I_{r}(\lambda)+\sqrt{I_{s}(\lambda) I_{r}(\lambda)}\left(e^{i \frac{2 \pi}{\lambda} \Delta O P L(t)}+e^{-i \frac{2 \pi}{\lambda} \Delta O P L(t)}\right)
$$

where $I_{s}$ and $I_{r}$ are the signal intensities from the sample arm and reference arm, respectively. 


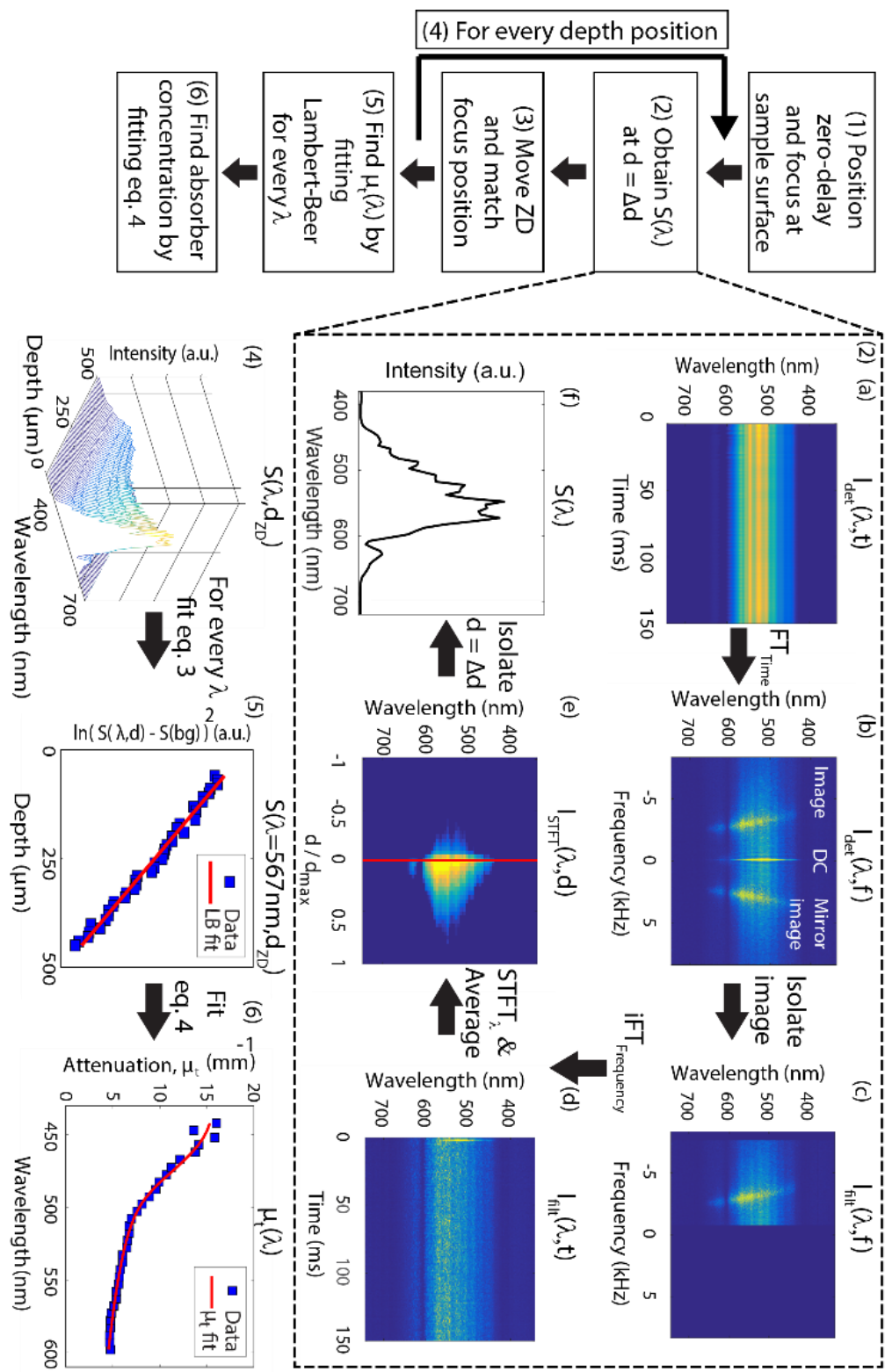

Figure 2.2: Schematic overview of the data acquisition and processing as performed in this study. All numbers and letters within parentheses denote the data processing steps that are explained in section 2.2.2. For all datasets containing complex data $(2 b-d)$, only the absolute values are shown. ZD: zerodelay, LB: Lambert-Beer. 


\subsubsection{Zero-delay acquisition}

The finite spectral resolution of the detecting spectrograph results in a sensitivity roll-off as a function of depth [14]. To optimize our system sensitivity, we apply zero-delay acquisition along the full axial range, i.e. at any depth inside the sample. Hereto, we apply the method described in our previous work to remove the undesired crosstalk between the OCT signal and its mirror image [15]. In short, a piezo-driven saw-tooth movement of the reference mirror induces a modulation of $\triangle O P L$ as a function of time with frequency $f_{D}=2 v_{r} / \lambda$, where $v_{r}$ is the velocity of the moving reference mirror $(0.85 \mathrm{~mm} / \mathrm{s})$. The change in $\triangle O P L$ is limited to $5 \mu \mathrm{m}$ by acquisition of only 50 lines (exposure time: $50 \mu \mathrm{s}$ per line, linerate: $16.7 \mathrm{kHz}$ ) per period of the piezo-driven movement (100 ms). Triggering of the camera by reference mirror position ensures that for every period, acquisition is performed over the same part (at which the movement speed is constant) of the piezo-driven movement. Acquisition of a total of $\mathrm{N}=$ 2500 lines takes 5 seconds and results in a dataset $I_{\text {det }}(\lambda, t)$ (Figure 2.2, step 2a). Fourier transformation of $I_{\text {det }}(\lambda, t)$ with respect to time results in the frequency content of the signal $I_{\text {det }}(\lambda, f)$, containing the frequency of the modulation of $\triangle O P L$ as a result of the reference mirror movement (Figure 2 , step $2 \mathrm{~b}$ ). The DC component and the positively shifted frequencies (mirror-image) are removed from $I_{\text {det }}(\lambda, f)$ by a rectangular bandpass filter around the negatively shifted frequencies $(-7.3$ to $-0.4 \mathrm{kHz}$ ) (Figure 2.2, step 2c). Inverse Fourier transformation of the filtered frequency content with respect to frequency results in the filtered intensity $l_{\text {fitt }}(\lambda, t)$, with the DC component and mirror image removed (Figure 2.2, step 2d):

$$
I_{\text {filt }}(\lambda, t) \propto \sqrt{I_{s}(\lambda) I_{r}(\lambda)} e^{-i \frac{2 \pi}{\lambda} \Delta O P L(t)}
$$

\subsubsection{Short time Fourier transformation}

For the purpose of quantitative and spatially confined spectroscopy, we are interested in $l_{s}$ as a function of both wavelength and geometrical depth $d$, with $d=\Delta O P L /(2 n)$. Since $I_{\text {fitt }}(\lambda, t)$ contains the spectral content of the OCT signal over the entire imaging range of the spectrograph $\left(d_{\max }=622 \mu \mathrm{m}\right)$, the spectra are not yet spatially confined at each depth. Short time Fourier transformation (STFT) of $I_{\text {fit }}(\lambda, t)$ with respect to $\lambda$ with a rectangular spectral window with a size of $\Delta \lambda=5 \mathrm{~nm}$, results in a depth-resolved spectral dataset $I_{\text {STFT }}(\lambda, d, t)$ with a spatial 
resolution $\Delta d=\lambda^{2} /(2 \mathrm{n} \Delta \lambda)$ ranging between $14 \mu \mathrm{m}$ (at $\lambda=440 \mathrm{~nm}$ ) and $27 \mu \mathrm{m}$ (at $\lambda=600 \mathrm{~nm}$ ). The STFT is applied directly in the wavelength domain, since an interpolation filter to convert data to the equidistant wave number domain has its own frequency characteristics which affect depth information and thus affects quantitative analysis. Averaging of $\left|I_{\text {STFT }}(\lambda, d, t)\right|$ with respect to time (i.e. for every line captured by the camera) yields $I_{\text {STFT }}(\lambda, d)$ (Figure 2.2, step 2e). Since $I_{r}$ remains constant during the complete measurement, $I_{\text {STFT }}(\lambda, d)$ contains the depth profile of $\sqrt{I_{s}}$ per wavelength, from which we obtain $S(\lambda)=I_{\text {STFT }}(\lambda, d=\Delta d)$ (Figure 2.2, step 2f). The backscattered spectrum at $d=\Delta d$ is used, since it has a better signal-to-noise ratio compared to the backscattered spectrum at $d=0$ (i.e. exactly at zero-delay), due to small remainders of the DC term after filtering.

\subsubsection{Focus tracking and depth-resolved acquisition}

In addition to zero-delay acquisition, we further optimize our system sensitivity by focus tracking. At the start of each measurement, the zero-delay and focus position are matched at the interface between the cuvette wall and the sample (Figure 2.2, step 1). Subsequently, $S\left(\lambda, d_{z D}\right)$ is obtained by acquisition of $S(\lambda)$ as a function of the zero-delay (ZD) position relative to the sample's surface $d_{z D}$, with step size $d x$ and the method described in sections 2.2.2.1 and 2.2.2.2. Hereto, the reference arm is elongated with steps of $d x+n$ and focus tracking is achieved by translating the sample lens with steps $d x / n$ towards the sample (Figure 2.2, step 3 ). This method for combined zero-delay acquisition and focus tracking results in a depth resolved and wavelength resolved OCT signal $S\left(\lambda, d_{z D}\right)$, from which the attenuation in depth only depends on the optical properties of the sample (Figure 2.2, step 4).

\subsubsection{Spectra of optical properties}

Under the assumption of single scattering, the attenuation coefficient $\mu_{t}(\lambda)$ of the sample is obtained by fitting a linear Lambert-Beer model to the natural logarithm of $S\left(\lambda, d_{z D}\right)$ (Figure 2.2, step 5):

$$
\ln \left(\left(S\left(\lambda, d_{Z D}\right)-S_{b g}\right)^{2}\right)=\ln (\alpha(\lambda))-2 \mu_{t}(\lambda) d_{Z D}
$$


with $\alpha(\lambda)$ and $\mu_{t}(\lambda)$ free running fit parameters, and $S_{b g}$ a background term that is obtained at a depth of $1000 \mu \mathrm{m}$ inside the non-scattering back wall of the sample containing cuvette.

The individual contributions of the scattering coefficient $\mu_{s}(\lambda)$ (modeled as a scatter power function $\left.a \lambda^{-b}\right)$ and the absorption coefficient $\mu_{a}(\lambda)$ to the total sample attenuation $\mu_{t}(\lambda)$ are obtained by fitting equation 2.4 to $\mu_{t}(\lambda)$, according to our method in [12] (Figure 2.2, step 6):

$$
\mu_{t}(\lambda)=a \lambda^{-b}+\sum C_{i} \mu_{a, i}(\lambda)
$$

with fit parameters: scaling factor $a$, scatter power $b$, and $C_{i}$ the concentration of the $i^{\text {th }}$ chromophore relative to the chromophore concentration at which the reference absorption spectrum $\mu_{a, i}(\lambda)$ was measured. The lower boundaries of all fit parameters were set to 0 . We obtained all $\mu_{a, i}(\lambda)$ by transmission spectroscopy (UV-2401PC, Shimadzu, Japan), comprising the absorption spectra for free bilirubin and albumin-bound bilirubin.

To validate the measured attenuation spectra, the theoretical Mie scattering coefficient spectrum [16] is calculated and compared to the attenuation spectra as measured by our system.

\subsubsection{Tissue mimicking samples}

To validate our method, we created a series of aqueous samples $(n=1.35)$ mimicking neonatal skin ( $\mu_{s} \approx 9 \mathrm{~mm}^{-1}$, assuming an anisotropy of $\left.g=0.8\right)$ [17] containing NIST certified polystyrene spheres $(400 \mathrm{~nm}$ diameter, $2.5 \mathrm{mg} / \mathrm{ml}$ solution, Thermo Scientific, USA), a physiological concentration of $40 \mathrm{mg} / \mathrm{ml}$ bovine serum albumin (Sigma-Aldrich, Germany), and bilirubin concentrations varying between 0-650 $\mu \mathrm{M}$ (Sigma-Aldrich, Germany). Bilirubin was dissolved in DMSO, resulting in a $14 \mathrm{mM}$ stock solution, prior to adding it to the rest of the sample. A $200 \mathrm{mM}$ Tris $\mathrm{HCl}(\mathrm{pH}=8.4)$ solution was used to dilute the bilirubin to its final concentration. The samples were measured over a depth interval of $d=0-500 \mu \mathrm{m}$ inside the sample $(d x=10 \mu \mathrm{m})$.

To demonstrate the spatially confined aspect of our SOCT method and to show that the sensitivity of our system is also sufficient to obtain accurate results at larger probing depths, we measured the attenuation of a $335 \mu \mathrm{M}$ bilirubinpolystyrene sample over a depth interval of 0-200 $\mu \mathrm{m}(d x=2 \mu \mathrm{m})$ behind a 
a

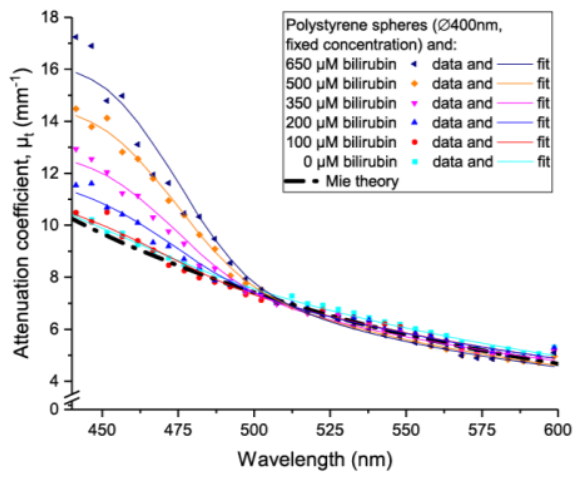

b

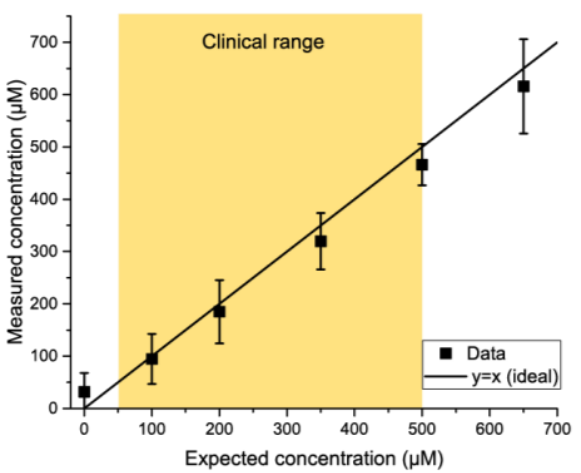

Figure 2.3: a. Average attenuation spectra $\mu_{t}(\lambda)$ for the samples with a fixed concentration of polystyrene spheres and varying bilirubin concentrations in the clinical pathophysiological range. Mie theory was used to estimate the theoretical scattering coefficient spectrum. Fits are obtained by fitting equation 4 to $\mu_{t}(\lambda)$. Error bars ( $\pm 10 \%$ ) are not shown for visibility reasons. b. Estimated bilirubin concentrations (average of 3 measurements, error bars represent standard deviations). Except for the $0 \mu \mathrm{M}$ bilirubin sample, all averaged values agree within $10 \%$ to the expected values. The results cover the full clinical pathophysiological range (indicated by the yellow box).

scattering silicone layer containing $0.1 \% \mathrm{TiO}_{2}\left(n=1.47, \mu_{s} \approx 5 \mathrm{~mm}^{-1}\right.$, thickness $=$ $150 \mu \mathrm{m}$, optical density $\approx 0.33$ ), which mimics the optically homogeneous upper part of the neonatal dermis [17], or adult epidermal skin layer $\left(\mu_{\mathrm{s}} \approx 4-8 \mathrm{~mm}^{-1}\right.$, thickness $\approx 100-150 \mu \mathrm{m}$ ) [12].

\subsection{Results}

The measured attenuation spectra $\mu_{t}(\lambda)$ for the polystyrene/bilirubin samples are shown in Figure 2.3a (average of 3 measurements), along with the fits obtained by fitting Eq. 2.4 to $\mu_{t}(\lambda)$. The attenuation for wavelengths that are absorbed by bilirubin $(\lambda<525 \mathrm{~nm})$ increases linearly with bilirubin concentration. The theoretical Mie scattering coefficient spectrum of the polystyrene spheres (dash-dotted black line in Figure 2.3a) is in excellent agreement with the non-absorbing $0 \mu \mathrm{M}$ bilirubin sample, as well as the other samples for $\lambda>525 \mathrm{~nm}$. 

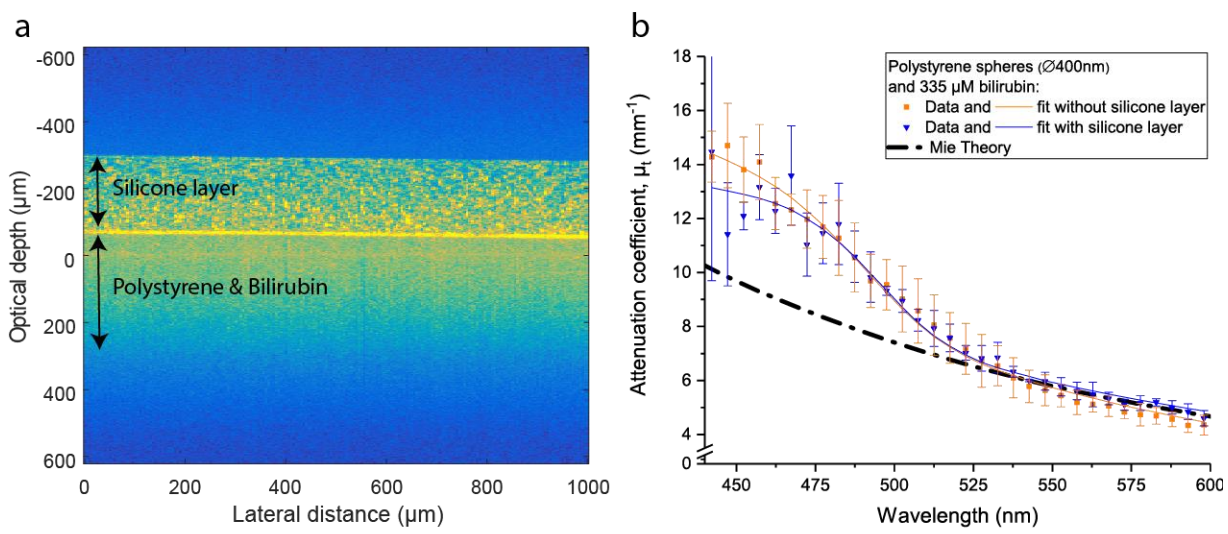

Figure 2.4: a. OCT image used to discriminate between the silicone layer and the sample. $b$. Attenuation spectra $\mu_{t}(\lambda)$ for the $335 \mu M$ bilirubin sample with and without the silicone layer (average of 3 measurements). Fits are obtained by fitting Eq. 2.4 to $\mu_{t}(\lambda)$. Mie theory was used to estimate the theoretical scattering coefficient spectrum.

Figure 2.3b shows the bilirubin concentrations that we derived by fitting Eq. 2.4 to the measured $\mu_{t}(\lambda)$. Except for the $0 \mu \mathrm{M}$ bilirubin sample, all average values agree within $10 \%$ with the expected values.

Figure 2.4a shows the OCT image of a $335 \mu \mathrm{M}$ bilirubin-polystyrene sample behind the scattering $\mathrm{TiO}_{2}$-silicone layer. From this image, we determined the depth of the bilirubin phantom layer, after which the optical attenuation spectrum was obtained using the same procedure of depth acquisition and temporal averaging as for the non-layered phantoms. The sample's attenuation spectrum $\mu_{t}(\lambda)$ was measured with and without coverage by the silicone layer, as shown in Figure 2.4b (average of 3 measurements). For $\lambda>460 \mathrm{~nm}$, the measured attenuation spectrum behind the silicone layer is in good agreement with the measured attenuation spectrum of the sample without the silicone layer. Again, both attenuation spectra show excellent agreement with the theoretical Mie scattering spectrum for $\lambda>525 \mathrm{~nm}$.

Fitting Eq. 2.4 to the measured attenuation spectra (solid lines, Figure 2.4b) results in bilirubin concentrations of $310 \pm 41 \mu \mathrm{M}$ and $326 \pm 46 \mu \mathrm{M}$ (mean \pm std of 3 measurements) with, and without coverage by the silicone layer, 
respectively. Both concentrations agree within $10 \%$ with the expected bilirubin concentration of $335 \mu \mathrm{M}$.

\subsection{Discussion and conclusion}

In this study, we have demonstrated the potential of visible-light SOCT to quantify bilirubin concentrations over the complete clinical pathophysiological range $(50-500 \mu \mathrm{M})$ in neonatal skin mimicking samples. Besides a good agreement between the measured and expected bilirubin absorption of our samples, we also obtained good agreement between the measured optical scattering and Mie theory. With that, we have taken the first steps towards spatially confined measurements of bilirubin concentrations in tissue.

At the bilirubin absorption peak around $460 \mathrm{~nm}$, our sOCT method effectively overcomes the problem of a sharp roll-off of the system sensitivity with depth $(-13 \mathrm{~dB}$ for the spectrograph's sensitivity at a depth of $500 \mu \mathrm{m}$ in a sample with $\mathrm{n}=1.35$ ), by applying zero-delay acquisition and focus tracking. Compared to other STFT-based methods for SOCT [6-11], our technique has the disadvantage of an increased measurement time. Our method requires acquisition of sufficient ( $\sim 50)$ lines to sample the movement of the piezo driven reference mirror, hence measurement time is limited by the reference mirror speed. The measurement time of our method also scales linearly with the depth interval over which we derive the attenuation coefficient by fitting Eq. 2.3. In this study, data was acquired at a minimum of 50 depth positions, resulting in a minimum measurement time of 250 seconds for a full dataset. For potential future in vivo measurements, it is likely that motion artefacts occur within this measurement time, hampering both the quantitative, and localized analysis of the optical attenuation. Measurement time can be reduced by a) reducing the period of the reference mirror movement, b) decreasing the number of averages per depth position, and c) increasing axial step size $d x$ or, depending on the application, reducing the depth range to a spatially even more confined region of interest. Options b) and c) potentially come at the cost of measurement accuracy.

For the sample measurement behind the scattering silicone layer, the measured $\mu_{t}(\lambda)$ is lower and has larger standard deviations than the $\mu_{t}(\lambda)$ measured without the layer for wavelengths $<460 \mathrm{~nm}$ (Figure $2.4 \mathrm{~b}$ ). This can be caused by a) a lower signal to noise ratio inside the sample, due to the increased 
attenuation by the scattering silicone layer in this spectral region, and b) the influence of multiple scattering on the signal at increasing measurement depths. The presence of multiple scattering results in a lower scattering contribution when fitting Eq. 2.4 to the measured $\mu_{t}(\lambda)$ [16]. Since absorption is not affected by the influence of multiple scattering [5], we still obtain approximately the same absorption contribution - and thus bilirubin concentration - compared to the measurement without the covering layer. Nevertheless, when translating this method to spatially confined measurements in tissue, a thorough investigation is required of the measurement accuracy as a function of depth and tissue optical density.

For all samples containing bilirubin, the averaged bilirubin concentration measurements agree within $10 \%$ of the expected values. The overestimation of the bilirubin concentration in the $0 \mu \mathrm{M}$ bilirubin sample may be explained by the lower boundary of 0 for all fit parameters in Eq. 2.4, resulting in a positive bias for samples containing no or very little bilirubin. Since the standard deviation of the data is in the order of $50 \mu \mathrm{M}$, the precision of our method is lower than its accuracy. This precision is comparable to the precision of transcutaneous bilirubinometers for concentrations up to $200 \mu \mathrm{M}$ [3]. Transcutaneous bilirubinometers however, systematically underestimate the clinically relevant bilirubin concentrations higher than $200 \mu \mathrm{M}$ [3], whereas the accuracy of our method remains relatively constant for the full investigated range. Furthermore, SOCT has the ability of spatially confining the measurement volume to a small region of interest inside tissue. This can be of great value to I) study the physiological process of bilirubin extravasation into tissue, and II) noninvasively measure bilirubin concentrations inside blood vessels. The latter would overcome the intrinsic accuracy-limiting factor of current transcutaneous bilirubinometers, as these measure the bilirubin concentration in a relatively large skin volume, which does not correlate directly to the bilirubin concentration in blood [2]. Further research is required to investigate whether SOCT can accurately measure bilirubin concentrations in blood where new challenges such as tissue dynamics, especially perfusion, and high hemoglobin absorption $\left(\mu_{a} \approx 20 \mathrm{~mm}^{-1}\right)$ around the same wavelengths as the bilirubin absorption peak arise. 


\section{Bibliography}

1. T. W. Hansen, "Prevention of neurodevelopmental sequelae of jaundice in the newborn," Dev Med Child Neurol 53(Suppl 4), 24-28 (2011).

2. N. Bosschaart, J. H. Kok, A. M. Newsum, D. M. Ouweneel, R. Mentink, T. G. van Leeuwen, and M. C. Aalders, "Limitations and opportunities of transcutaneous bilirubin measurements," Pediatrics 129(4), 689-694 (2012).

3. K. Grohmann, M. Roser, B. Rolinski, I. Kadow, C. Muller, A. GoerlachGraw, M. Nauck, and H. Kuster, "Bilirubin measurement for neonates: Comparison of 9 frequently used methods," Pediatrics 117(4), 11741183 (2006).

4. A. Knudsen, "The Cephalocaudal Progression of Jaundice in Newborns in Relation to the Transfer of Bilirubin from Plasma to Skin," Early Hum Dev 22(1), 23-28 (1990).

5. N. Bosschaart, M. C. G. Aalders, D. J. Faber, J. J. A. Weda, M. J. C. van Gemert, and T. G. van Leeuwen, "Quantitative measurements of absorption spectra in scattering media by low-coherence spectroscopy," Opt Lett 34(23), 3746-3748 (2009).

6. F. E. Robles and A. Wax, "Separating the scattering and absorption coefficients using the real and imaginary parts of the refractive index with low-coherence interferometry," Opt Lett 35(17), 2843-2845 (2010).

7. J. Yi, Q. Wei, W. Liu, V. Backman, and H. F. Zhang, "Visible-light optical coherence tomography for retinal oximetry," Opt Lett 38(11), 17961798 (2013).

8. J. Yi, W. Z. Liu, S. Y. Chen, V. Backman, N. Sheibani, C. M. Sorenson, A. A. Fawzi, R. A. Linsenmeier, and H. F. Zhang, "Visible light optical coherence tomography measures retinal oxygen metabolic response to systemic oxygenation," Light-Sci Appl 4, e334 (2015).

9. S. P. Chong, C. W. Merkle, C. Leahy, H. Radhakrishnan, and V. J. Srinivasan, "Quantitative microvascular hemoglobin mapping using visible light spectroscopic Optical Coherence Tomography," Biomed. Opt. Express 6(4), 1429-1450 (2015).

10. F. E. Robles, C. Wilson, G. Grant, and A. Wax, "Molecular imaging truecolour spectroscopic optical coherence tomography," Nat Photonics 5(12), 744-747 (2011). 
11. S. Y. Chen, X. Shu, J. Yi, A. Fawzi, and H. F. Zhang, "Dual-band optical coherence tomography using a single supercontinuum laser source," J Biomed Opt 21(6), 066013 (2016).

12. N. Bosschaart, D. J. Faber, T. G. van Leeuwen, and M. C. G. Aalders, "In vivo low-coherence spectroscopic measurements of local hemoglobin absorption spectra in human skin," J Biomed Opt 16(10), 100504 (2011).

13. S. Pi, A. Camino, W. Cepurna, X. Wei, M. Zhang, D. Huang, J. Morrison, and $Y$. Jia, "Automated spectroscopic retinal oximetry with visible-light optical coherence tomography," Biomed. Opt. Express 9(5), 2056-2067 (2018).

14. S. Yun, G. Tearney, B. Bouma, B. Park, and J. de Boer, "High-speed spectral-domain optical coherence tomography at 1.3 mum wavelength," Opt Express 11(26), 3598-3604 (2003).

15. N. Bosschaart, M. C. Aalders, T. G. van Leeuwen, and D. J. Faber, "Spectral domain detection in low-coherence spectroscopy," Biomed Opt Express 3(9), 2263-2272 (2012).

16. N. Bosschaart, D. J. Faber, T. G. van Leeuwen, and M. C. G. Aalders, "Measurements of wavelength dependent scattering and backscattering coefficients by low-coherence spectroscopy," J Biomed Opt 16(3), 030503 (2011).

17. N. Bosschaart, R. Mentink, J. H. Kok, T. G. van Leeuwen, and M. C. G. Aalders, "Optical properties of neonatal skin measured in vivo as a function of age and skin pigmentation," J Biomed Opt 16(9), 097003 (2011). 


\title{
Chapter 3:
}

\section{Quantification of total haemoglobin concentrations in human whole blood by spectroscopic visible-light optical coherence tomography}

\begin{abstract}
The non-invasive quantification of total haemoglobin concentrations [tHb] is highly desired for the assessment of haematologic disorders in vulnerable patient groups, but invasive blood sampling is still the gold standard in current clinical practice. This work demonstrates the potential of visible-light spectroscopic optical coherence tomography (sOCT) for quantifying the [tHb] in human whole blood. To accurately quantify the [tHb] from the substantial optical attenuation by blood in the visible wavelength range, we used a combination of zero-delay acquisition and focus tracking that ensures optimal system sensitivity at any depth inside the sample. Subsequently, we developed an analysis model to adequately correct for the high scattering contribution by red blood cells to the SOCT signal. We validate our method and compare it to conventional SOCT (without focus tracking and zero-delay acquisition) through ex-vivo measurements on flowing human whole blood, with [tHb] values in the clinical range of $7-23 \mathrm{~g} / \mathrm{dL}$. For our method with optimized sensitivity, the measured and expected values correlate well (Pearson correlation coefficient = $0.89, \mathrm{p}<0.01$ ), with a precision of $3.8 \mathrm{~g} / \mathrm{dL}$. This is a considerable improvement compared to conventional SOCT (Pearson correlation coefficient $=0.59, \mathrm{p}=$ 0.16 ; precision of $9.1 \mathrm{~g} / \mathrm{dL}$ ).
\end{abstract}

This chapter is published as: Colin Veenstra*, Saskia Kruitwagen*, Dafne Groener, Wilma Petersen, Wiendelt Steenbergen and Nienke Bosschaart, "Quantification of total haemoglobin concentrations in human whole blood by spectroscopic visible-light optical coherence tomography," Sci. Rep. 9, 15115 (2019)

*Both authors contributed equally. 


\subsection{Introduction}

Haemoglobin is the oxygen transporting protein in red blood cells (RBCs) and exists mainly in two forms: oxyhaemoglobin $\left(\mathrm{HbO}_{2}\right)$ and deoxyhaemoglobin $(\mathrm{Hb})$, which together account for the total amount of haemoglobin (tHb). Current clinical methods that measure total haemoglobin concentrations [tHb] in blood require invasive blood sampling, which are frequently used for diagnosing haematologic disorders such as anaemia and polycythaemia [1]. While invasive blood sampling is fast and highly accurate, it cannot be used for continuous monitoring for acute and intensive care purposes [2]. In premature infants, invasive blood sampling is associated with increased risk of infection and significant blood loss. For this vulnerable patient group, frequent blood sampling may in fact be the cause, rather than the prevention of anaemia [3]. Hence, there is an obvious need for the non-invasive assessment of [tHb].

Currently, non-invasive optical techniques are being developed for measuring [tHb], which make use of the high optical absorption of haemoglobin in the visible and near-infrared wavelength range. These techniques include photoplethysmography and photoacoustics. However, the precision of photoplethysmography is limited by the uncertainty in the exact optical path length of the detected photons [4], and the precision of photoacoustic [tHb] determinations remains to be determined [5]. Therefore, we follow an alternative approach by using visible-light spectroscopic optical coherence tomography (sOCT) for non-invasive quantification of [tHb]. The unique advantage of SOCT is that the optical path length (OPL) inside tissue is both known and controllable. This allows for both quantitative and spatially confined measurements of chromophore concentrations in-vivo [6], and potentially enables [tHb] determinations within individual blood vessels - without any cross-talk from the optical attenuation by surrounding tissue structures.

Visible light SOCT (vis-sOCT) and other low-coherent techniques have extensively proven their ability for haemoglobin oxygen saturation measurements [7-12] $\left(\mathrm{stO}_{2}=\left[\mathrm{HbO}_{2}\right] /[\mathrm{tHb}]\right)$. As the $\mathrm{stO}_{2}$ is based on the relative ratio between $\left[\mathrm{HbO}_{2}\right]$ and $[\mathrm{tHb}], \mathrm{stO}_{2}$ measurements primarily require adequate recovery of the shape of the absorption spectrum. On the other hand, the accurate quantification of [tHb] requires adequate recovery of the amplitude of the absorption spectrum. The latter is challenging in SOCT, as this requires precise correction for the scattering contribution to the measured attenuation, 
as well as the correction for system dependent attenuation parameters such as defocus and the sensitivity roll-off with depth due to the finite pixel size of the detecting spectrograph [13]. Moreover, superior system sensitivity in depth is required to adequately quantify the large optical attenuation of whole blood. As a consequence, current efforts for quantitative [tHb] measurements with SOCT have remained restricted to low haemoglobin concentrations in phantoms [14] $([\mathrm{tHb}]<6 \mathrm{~g} / \mathrm{dL})$ and limited validation on rodent whole blood in terms of sample size and reference methods [8]. These results indicate the great potential of vis-sOCT for the quantification of [ $\mathrm{tHb}$ ], but proof of principle across the clinical [tHb] range of 7-23 g/dL in human whole blood [15] remains to be given.

Recently, we developed a visible-light SOCT system with superior sensitivity by employing focus tracking and zero-delay acquisition [16] throughout the entire imaging depth, and we demonstrated its efficacy for the quantification of bilirubin concentrations [13]. In this study, we employ this SOCT system for quantitative $[\mathrm{tHb}]$ measurements on human whole blood and compare the results with conventional SOCT, i.e. without focus tracking and zero-delay acquisition. An analysis model is developed to adequately correct for the high scattering contribution by red blood cells to the SOCT signal. We validate our method on human whole blood samples with $[\mathrm{tHb}]$ concentrations ranging between $7-23 \mathrm{~g} / \mathrm{dL}$.

\subsection{Results}

\subsubsection{Attenuation coefficient spectra}

Human whole blood samples with varying [tHb] were studied with our visiblelight SOCT system to obtain the attenuation coefficient $\mu_{t}$ as a function of wavelength $\lambda$. This $\mu_{t}(\lambda)$ is the sum of the absorption coefficient $\mu_{a}(\lambda)$ and the scattering contribution to the signal, which equals the scattering coefficient $\mu_{s}(\lambda)$ in the case of single scattering. As an example, typical $\mu_{t}(\lambda)$ spectra obtained by both analysis methods are shown in Figure 3.1, along with the estimated contribution of scattering. For $\lambda<600 \mathrm{~nm}$, the measured $\mu_{t}(\lambda)$ typically increases with expected [tHb]. The attenuation model (eq. 3.5) fits better to the measured $\mu_{t}(\lambda)$ spectra that were acquired with focus tracking and zero-delay acquisition, with an average ( \pm standard deviation SD) coefficient of determination $\left(R^{2}\right)$ of $0.91 \pm 0.08$, versus $0.71 \pm 0.17$ for the conventional sOCT 
a)

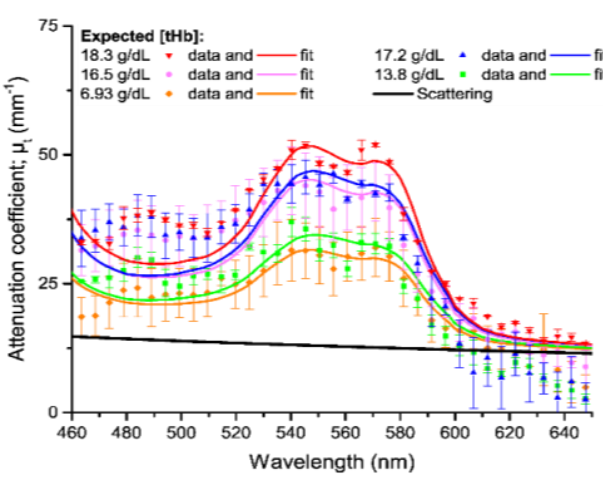

b) Focus tracking and zero-delay acquisition

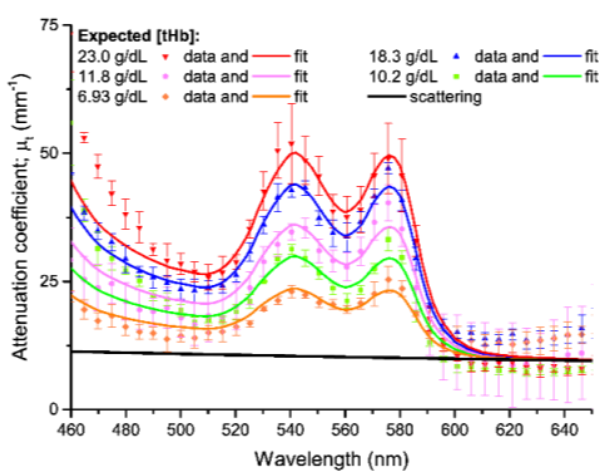

Figure 3.1: Example of measured and fitted attenuation spectra $\mu_{t}(\lambda)$ for samples with varying [tHb] (average spectrum per sample, error bars represent $S D)$. The plotted scattering spectrum represents the average scattering contribution of all whole blood samples that were included in this study. a) Attenuation spectra obtained by conventional sOCT. b) Attenuation spectra obtained by combining focus tracking and zero-delay acquisition.

method. For $\lambda>600 \mathrm{~nm}$, scattering dominates absorption, resulting in a similar $\mu_{t}(\lambda)$ across all samples for the focus tracking \& zero-delay acquisition method

\subsubsection{Estimated haemoglobin concentrations}

\subsubsection{Conventional SOCT}

The measured [tHb] as a function of expected [tHb] is shown in Figure 3.2a. The measured and expected values are moderately correlated (Pearson correlation coefficient $=0.59, p=0.16$ ). Linear regression on the data results in $y=0.94 x+$ 2.25. Figure $3.2 \mathrm{~b}$ shows the difference between the measured and expected values as a function of the average of the measured and expected value (BlandAltman plot), from which we obtain a bias of $1.5 \mathrm{~g} / \mathrm{dL}$ and a precision of $9.1 \mathrm{~g} / \mathrm{dL}$ (1.96 SDs).

\subsubsection{Focus tracking and zero-delay acquisition}

Figure 3.2c shows the measured [tHb] (Eq. 3.5) as a function of expected [tHb]. The measured and expected values correlate well (Pearson correlation coefficient $=0.89, p<0.01$ ). Linear regression on the data results in $y=0.89 x+$ 
Conventional sOCT
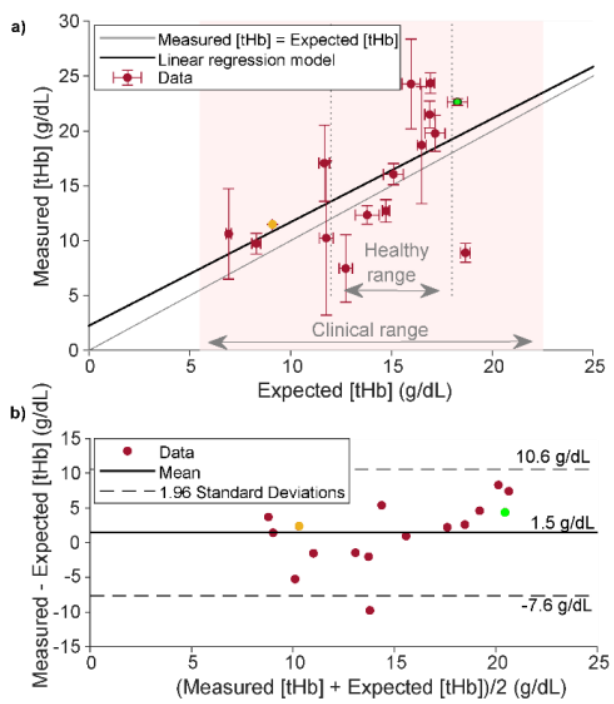

Focus tracking and zero-delay acquisition
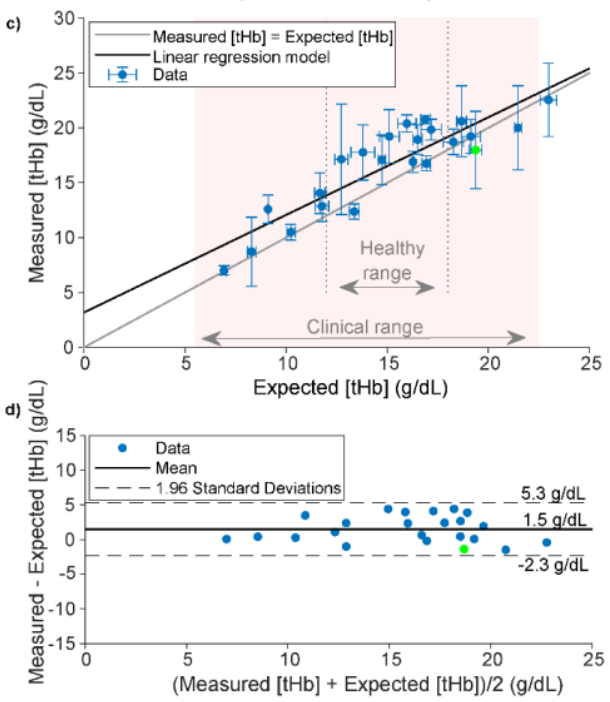

Figure 3.2: Measured and expected [tHb] values. a) Measured versus expected [tHb] values for all samples (average $\pm S D$ ) by using conventional sOCT $(n=16)$. b) A Bland-Altman plot for the conventional method shows a bias of $1.5 \mathrm{~g} / \mathrm{dL}$ and a precision of $9.1 \mathrm{~g} / \mathrm{dL}$ (1.96 SDs). c) Measured versus expected [tHb] values for all samples (average $\pm S D$ ) by combining focus tracking and zero-delay acquisition ( $n=23)$. d) The Bland-Altman plot of the data shows a bias of $1.5 \mathrm{~g} / \mathrm{dL}$ and a precision of $3.8 \mathrm{~g} / \mathrm{dL}$. An average of 3 measurements was taken and error bars represent SD. The data points in green were only measured in duplo, while the data point in orange represents a single measurement. As detailed in the methods section, not all samples could be included in the conventional sOCT analysis, due to an excessive loss of signal for the higher [tHb] values.

3.18. From the Bland-Altman plot (figure $3.2 \mathrm{~b}$ ) we obtain a bias of $1.5 \mathrm{~g} / \mathrm{dL}$ (mean) and a precision of $3.8 \mathrm{~g} / \mathrm{dL}$.

\subsection{Discussion}

In this study, we used sOCT to quantify the [tHb] in human whole blood throughout the clinical range. We compared our SOCT method with optimized sensitivity to conventional SOCT, and developed a model to adequately correct for the high scattering contribution of RBCs to the sOCT signal. 
In conventional SOCT, the backscattered intensity as a function of depth is not only affected by the sample, but also by the defocus and sensitivity roll-off of the system. Although a correction for roll-off and defocus can be applied to retrieve the sample's attenuation coefficient, this does not omit the decrease in signal to noise ratio with depth. Our SOCT system overcomes this problem by combining focus tracking with zero-delay acquisition. Compared to conventional SOCT, our method yielded an improved correlation between the attenuation spectra (Figure 3.1) and the attenuation model (Eq. 3.5), and demonstrated improved precision for the [tHb] estimations (Figure 3.2). Whereas the conventional method failed to retrieve usable attenuation spectra for those samples with the highest absorption ([tHb] $>19 \mathrm{~g} / \mathrm{dL}$ ), the improved sensitivity of the focus tracking and zero-delay acquisition method resulted in usable data points in this region. We would like to note that the presented comparison is system dependent, as it will be different for SOCT systems with other roll-off and defocus characteristics.

In our comparison to conventional SOCT, we followed a general approach to correct for the OCT system-dependent attenuation in depth [17]. Another method described in literature for in vivo whole blood SOCT measurements is division of the backscattered signal inside a blood vessel by the backscattered signal from a certain volume adjacent to that vesse $l^{8}$. Whereas this method may be sensitive to tissue inhomogeneities, the ex vivo nature of our data did not allow for comparison in this study.

The scattering contribution of blood as determined by our method $\left(\sim 10 \mathrm{~mm}^{-1}\right)$ is many times lower than literature values $\left(70-120 \mathrm{~mm}^{-1}\right)$ [18]. This can be ascribed to the highly forward directed scattering of RBCs, which causes a large contribution of multiple scattered photons to the OCT-signal. This contribution violates the assumption of single scattering in our Lambert-Beer model (Eq. 3.1), thereby effectively lowering the measured attenuation [17]. As a consequence, the measured scattering contribution in our measurements is lower than the actual scattering coefficient of whole blood. As the measured scattering contribution by OCT tends to become less dependent on particle concentration for high concentrations [17], this multiple scattering effect further strengthens our assumption of a constant scattering contribution for all blood samples [18]. As long as the estimation of this scattering contribution to the measured attenuation spectrum is adequate, the estimated absorption coefficient - and 
thus [tHb] - remain unaffected by multiple scattering: absorption takes place along the photon's controlled path, which is identical for both single and multiple scattered photons within the coherence length of the SOCT system [19].

The complexity of flowing human whole blood may influence its optical properties and therefore the precision our [tHb] estimations [18]. For this study, the precision of the $[\mathrm{tHb}]$ determinations by SOCT is lower than currently available invasive [tHb] measurement methods and photoplethysmography based non-invasive methods ( $2 \mathrm{~g} / \mathrm{dL}$ based on $1.96 \mathrm{SDs}$ ) [4]. There are several reasons for this impaired precision, which may provide room for improvement in future work. Due to the non-Newtonian behaviour of blood, blood flow can induce a non-homogeneous distribution of red blood cells in the radial direction of the capillary. The low shear rate near the wall gives rise to a cell free layer, while an accumulation of cells occurs in the central zone of the capillary [20]. Also aggregates of red blood cells (e.g. rouleaux) can be formed in this central zone of the capillary, and sedimentation due to gravity may occur. All of these effects contribute to the non-homogeneous distribution of blood absorption across the capillary cross section [20], which will directly affect the measured [tHb]. In this study, we accounted for the cell free layer by excluding the first 15 $\mu \mathrm{m}$ relative to the capillary-sample interface from the measurements. To minimise the effect of the non-uniform distribution of haemoglobin through the capillary, the attenuation coefficient is obtained by fitting Beer's law (eq. 3.1) over a confined depth range of $15-55 \mu \mathrm{m}$ relative to the capillary-sample interface. As the capillary flow profile can potentially be derived from the OCTsignal directly [21], this will be an interesting topic for future studies focusing on improved precision of the [tHb] determination. Also other factors of influence, such as vessel depth, vessel diameter and optical density of the tissue covering the vessel, will be important to evaluate.

Although our method of zero-delay acquisition and focus tracking results in optimal sensitivity as a function of measurement depth, it comes at the cost of measurement time. Acquisition of the backscattered spectrum at zero-delay at every depth in the sample requires acquisition of sufficient ( $\sim 50)$ lines in order to sample the movement of the piezo driven reference mirror at every depth position. As a consequence, the measurement time scales linearly with the depth interval over which $\mu_{t}$ is measured. In this work, 20 depth positions were 
used, resulting in a measurement time of 100 seconds per sample. Future invivo applications require a reduction of measurement time, which can be achieved through optimization of the scanning velocity of the reference mirror, the camera's line rate (limited by the exposure time), the choice of the depth range and the number of averages, potentially reducing the measurement time by a factor 20 . While the first option can be introduced without reducing the signal-to-noise ratio (SNR), the other options will likely reduce the SNR of the system, which may in turn reduce the precision of the [tHb] estimations. Further research is necessary to find an optimal location on the body for future in-vivo measurements. Taking the cell free layer into account, blood vessels with a diameter of $\geq 70 \mu \mathrm{m}$ will be ideal for in-vivo [tHb] determinations. Our method will presumably perform best at a body location where these vessels run as superficial as possible to the skin surface, with low susceptibility for movement artefacts.

\subsection{Conclusion}

In conclusion, this ex-vivo study demonstrates proof of principle for [tHb] measurements by SOCT in human whole blood throughout the clinical range of $7-23 \mathrm{~g} / \mathrm{dL}$. With a correlation coefficient of 0.89 between the measured and expected [tHb], sOCT has potential to become a non-invasive alternative for invasive [tHb] determinations, for which future in-vivo validation studies will be necessary.

\subsection{Methods}

\subsubsection{Experimental setup}

The SOCT system and data processing have been introduced in detail in Veenstra, et al. [13] and Bosschaart, et al. [16], and will be briefly described next. Compared to our previous work [13], the SOCT system alignment was modified to increase sensitivity for the longer wavelengths $(\lambda>600 \mathrm{~nm})$ within the visible wavelength range at the cost of sensitivity for shorter wavelengths $(\lambda<460 \mathrm{~nm}$ ). The sOCT system (Figure. 3.3 ) had the possibility to acquire the OCT signal as a function of depth with focus tracking and zero-delay acquisition [16]. This optimises system sensitivity and to allows for measurements of the sample's optical attenuation coefficient $\mu_{t}$ independent of system parameters [13]. The light of a supercontinuum light source (SuperK EXTREME EXB-6, NKT Photonics, Denmark) was short pass filtered at $700 \mathrm{~nm}$ (FESH0700, Thorlabs, 
USA) after which a beam splitter (BS028, Thorlabs, USA) guided $90 \%$ and $10 \%$ of the light towards the reference and sample arm, respectively. Achromatic lenses (AC127-025-A, Thorlabs, USA) focused the light on the sample contained by a glass capillary in the sample arm (Fisher Scientific B.V., The Netherlands, inner diameter $1.2 \mathrm{~mm}$, outer diameter $1.8 \mathrm{~mm}$ ), and a piezo-driven oscillating mirror in the reference arm. The Rayleigh length of the foci was $55 \mu \mathrm{m}$ at $\lambda=$ $550 \mathrm{~nm}$. The optical power incident on the sample was $\sim 6 \mathrm{~mW}$. A motorised linear stage (T-LS13M, Zaber, USA) controlled the reference arm length by joint translation of the reference mirror and its focusing lens. An identical stage facilitated focus tracking by translation of the focusing lens in the sample arm. The back scattered light from both arms was guided by a single mode fibre (S405-XP, Thorlabs, USA) to a home-built spectrometer, where a grating dispersed the light onto a line scan camera (Sprint spL4096-140km, Basler, Germany) resulting in spectral resolution $\delta \lambda=0.1 \mathrm{~nm}$. The detected spectrum ranged from 460 to $650 \mathrm{~nm}$, resulting in a coherence length of $1.4 \mu \mathrm{m}$ in air. The sensitivity of the system was estimated at $104.7 \mathrm{~dB}$, with an $-3 \mathrm{~dB}$ sensitivity rolloff depth of $307 \mu \mathrm{m}$ when zero-delay acquisition was not applied.

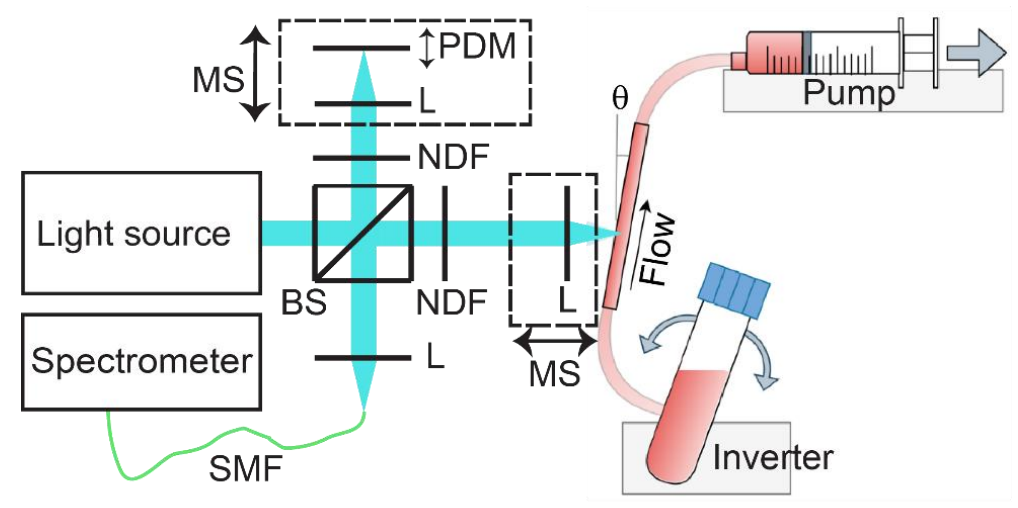

Figure 3.3: Schematic overview of a blood sample flowing through the sample arm of the sOCT setup. A blood containing tube is placed on an inverter, and blood flows through a glass capillary in which the measurement takes place. $A$ continuous flow is established by a syringe pump. The light is focused inside the capillary by a lens placed on a motorised stage that allows for focus tracking inside the sample. BS: beam splitter, NDF: neutral density filter, L: lens, PDM: piezo driven mirror, MS: motorised stage, SMF: single mode fibre, $\theta: 10$ degree angle. 


\subsubsection{Focus tracking and zero-delay acquisition}

Due to the finite pixel size of the detector, SOCT comes with a strong sensitivity roll-off as a function of optical path length difference ( $\triangle O P L$ ) between both arms, especially in the visible wavelength region. To acquire the OCT-signal with maximum spectrograph sensitivity across all depths, we performed acquisition at $\triangle O P L=0$ (zero-delay) at any depth inside the sample. As a consequence, the unwanted crosstalk between the OCT-signal, its complex conjugate and the DCcomponent around zero-delay needed to be removed. We followed the same approach as in our previous work $[13,16]$, where the piezo-driven oscillating reference mirror modulated the optical path length difference between the sample and reference arm, resulting in a Doppler shift with frequency $f_{d}=2 v_{r} / \lambda$. Here $v_{r}=0.85 \mathrm{~mm} / \mathrm{s}$ was the velocity of the reference mirror and the spatial scanning range of the mirror was $5 \mu \mathrm{m}$ [13]. By applying a rectangular bandpass filter around the negatively shifted frequencies in the Fourier-domain (-7.3 to $0.4 \mathrm{kHz}$ ), the DC-component and the complex conjugate could be effectively removed from the OCT-signal [13].

\subsubsection{Short time Fourier transformation}

Prior to each measurement, zero-delay and focus position were matched at a depth of $15 \mu \mathrm{m}$ relative to the sample-capillary interface. Spectroscopic detection of a total of $N=2500$ lines (exposure time: $50 \mu$ s per line, line rate: $16.7 \mathrm{kHz}$ ) was performed. Next, short time Fourier transformation resulted in a spatially and spectrally confined dataset (spectral resolution $5 \mathrm{~nm}$, spatial resolution ranging between $15 \mu \mathrm{m}$ at $460 \mathrm{~nm}$ and $30 \mu \mathrm{m}$ at $650 \mathrm{~nm}$ ) from which the backscattered spectrum at zero-delay $S(\lambda)$ could be isolated using the procedure described above. Translation of both zero-delay and focus position by the motorised stages allowed for acquisition of $S(\lambda)$ at any depth. This procedure was repeated until the complete desired depth range was covered, resulting in a spectrally and spatially resolved dataset of backscattered light from the sample $S(\lambda, d)$.

\subsubsection{Retrieving the attenuation spectrum}

Prior to attenuation analysis, $S_{\text {conv }}(\lambda, d)$ was corrected for the OCT-system dependent attenuation similar to the approach in Almasian, et al. [17] A wavelength dependent correction for the sensitivity roll-off of the system was 
applied by dividing $S_{\text {conv }}(\lambda, d)$ by the relative sensitivity of the system as a function of depth [22] for every wavelength. Under the assumption of single scattering, the attenuation coefficient $\mu_{t}(\lambda)$ of the sample was obtained by fitting a linear Lambert-Beer model to the natural logarithm of $S(\lambda, d)[13]$ :

$$
\ln \left(\left(S(\lambda, d)-S_{b g}(\lambda)\right)^{2}\right)=\ln (\alpha(\lambda))-2 \mu_{t}(\lambda) d
$$

with $\alpha(\lambda)$ and $\mu_{t}(\lambda)$ free running fit parameters and $S_{b g}(\lambda)$ a background term that was obtained by measuring $S(\lambda)$ at a depth of $1000 \mu \mathrm{m}$ inside the blood sample, where light had been fully attenuated.

\subsubsection{Conventional sOCT}

To investigate the advantages of our focus tracking and zero-delay acquisition method, we used a conventional SOCT method without reference mirror oscillation and focus tracking for comparison. To prevent the complex conjugate from interfering with the signal, the surface of the sample and the focus position were matched at a depth of $100 \mu \mathrm{m}$ relative to zero-delay. Similar to the method described above, spectroscopic detection of a total of $N=2500$ lines (exposure time: $50 \mu$ s per line, line rate: $16.7 \mathrm{kHz}$ ) was performed and the spectra were corrected for the background by subtracting the signal measured at a depth of $1000 \mu \mathrm{m}$ inside the sample. To ensure the most robust fitting procedure for the Lambert-Beer model (below) with the most stable outcome for $\mu_{t}(\lambda)$, short time Fourier transformation was performed with a larger spectral window $(20 \mathrm{~nm})$ compared to our focus tracking and zero-delay method $(5 \mathrm{~nm})$. This resulted in a spatially and spectrally resolved dataset $S_{\text {conv }}(\lambda, d)$ with a spatial resolution that ranged from $3.8 \mu \mathrm{m}$ at $460 \mathrm{~nm}$ to $7.5 \mu \mathrm{m}$ at $650 \mathrm{~nm}$. A 75\% overlap for the spectral window yielded an effective $5 \mathrm{~nm}$ spectral spacing between the data, identical to the focus tracking and zero-delay acquisition method.

\subsubsection{Retrieving the attenuation spectrum}

Correction for the sensitivity roll-off of the system was applied by dividing $S_{\text {conv }}(\lambda, d)$ by the relative sensitivity of the system as a function of depth [22] for every wavelength. Under the assumption of single scattering, the attenuation coefficient $\mu_{t}(\lambda)$ was obtained similar to Eq. 3.1 by fitting a Lambert-Beer model to the background corrected $S_{\text {conv }}(\lambda, d)$ : 


$$
\ln \left(S_{\text {conv }}(\lambda, d)^{2}\right)=\ln (\alpha(\lambda))-2 \mu_{t}(\lambda) d
$$

with $\alpha(\lambda)$ and $\mu_{t}(\lambda)$ free running fit parameters.

\subsubsection{Estimating chromophore concentrations}

For both SOCT methods, we modeled the obtained total attenuation coefficient as the sum of a scatter power function $a \lambda^{-b}$ and the individual absorption spectra of $\mathrm{HbO}_{2}$ and $\mathrm{Hb}$ in whole blood:

$$
\mu_{t}(\lambda)=a \lambda^{-b}+\sum_{i} C_{i} \mu_{a, i}(\lambda)
$$

with scaling factor $a$, scatter power $b$ and $C_{i}$ the concentration of the i-th chromophore relative to the reference absorption spectrum $\mu_{a, i}(\lambda)$.

In blood, the major fraction of haemoglobin is located inside RBCs rather than being homogeneously distributed throughout the sample. The fraction of light that does not encounter RBCs will not be affected by absorption, causing the absorption to scale non-linearly with concentration. This phenomenon is known as absorption flattening, and can be described by: $[18,23]$

$$
\mu_{a, \text { effective }}=\left(\frac{1-\exp \left(-\mu_{a, \text { free }} d_{r b c}\right)}{\mu_{a, \text { free }} d_{r b c}}\right) \mu_{a, \text { free }}
$$

where $\mu_{a, f r e e}$, is the absorption coefficient of an absorber when freely present in a solution and $\mu_{a, \text { effective }}$ the effective absorption coefficient if the absorber is located inside particles. In the derivation of Eq. 3.4 RBCs were modelled as cubes with edge length $d_{r b c}=\sqrt[3]{90 \mu \mathrm{m}^{18}}$. Combining Eq. 3.3 and Eq. 3.4 results in:

$$
\mu_{t}(\lambda)=a \lambda^{-b}+\sum_{i}\left(\frac{1-\exp \left(-C_{i} \mu_{a, i}(\lambda) d_{r b c}\right)}{d_{r b c}}\right)
$$

which is the model for the total attenuation as used throughout this work. The non-flattened reference spectra $\mu_{a, i}$ used in this work were calculated by solving Eq. 3.4 for $\mu_{a, f r e e}$ after inserting the (flattened) $\mathrm{HbO}_{2}$ and $\mathrm{Hb}$ absorption spectra in whole blood from Bosschaart et al. [18] as $\mu_{a, \text { effective. }}$ 
Due to the high amount of dependent scattering by RBCs, scattering can be assumed to be constant within the haematocrit range associated with the measured haemoglobin concentrations (hct $\approx 21-69 \%$ ) [18]. We therefore used a single set of scaling factor $a$ and scatter power $b$ to correct all measured $\mu_{t}(\lambda)$ for scattering. The values for $a$ and $b$ were obtained by non-linear least squares fitting of Eq. 3.5 to the average $\mu_{t}(\lambda)$ of all measurements in the $460-650 \mathrm{~nm}$ wavelength range. The lower and upper limits of all fit parameters $\left(a, b\right.$ and $\left.C_{i}\right)$ were set to 0 and infinity, respectively. This procedure resulted in an estimated contribution of scattering to the total attenuation modelled by $\mu_{\mathrm{s}}\left[\mathrm{mm}^{-1}\right]=$ $258.2 \lambda^{-0.5165}$, with $\lambda$ in $\mathrm{nm}$. This leads to a $\mu_{s}$ ranging from $9.1 \mathrm{~mm}^{-1}$ to $10.9 \mathrm{~mm}^{-}$ ${ }^{1}$ for the $460-650 \mathrm{~nm}$ wavelength range.

Chromophore concentrations were obtained by fitting Eq. 3.5 to $\mu_{t}(\lambda)$ in the wavelength range $520-590 \mathrm{~nm}$ for each measurement separately, with $a$ and $b$ as determined by the procedure in the previous paragraph and $C_{i}$ as the only fit parameter with limits 0 - infinity. The [tHb] was obtained by summing the individual contributions of $\left[\mathrm{HbO}_{2}\right]$ and $[\mathrm{Hb}]$.

\subsubsection{Human whole blood samples}

A $15 \mathrm{~mL}$ falcon tube filled with $9 \mathrm{~mL}$ blood sample was placed on an inverter (frequency $0.1 \mathrm{~Hz}$ ), to prevent sedimentation of RBCs and to ensure continuous mixing of the blood throughout each measurement. A syringe pump (AL1000220, World Precision Instruments, USA) established a blood flow speed of 0.3 $\mathrm{mL} / \mathrm{min}$ through the glass capillary, which was placed inside the sample arm. The capillary was placed under an angle of $\sim 10$ degrees relative to the incident beam to prevent detection of specular reflections from the glass capillary. Measurements were performed in a depth range of 15 - $55 \mu \mathrm{m}$ (step size $2 \mu \mathrm{m}$ ) relative to the capillary-sample interface.

To validate our focus tracking and zero-delay acquisition method, we created and measured a series of 23 blood samples with varying expected [tHb] in the range of $6.9-23.0 \mathrm{~g} / \mathrm{dL}$. For 18 of these blood samples ([tHb] from 6.9 to 21.5 $\mathrm{g} / \mathrm{dL}$ ), the OCT signal was also acquired without reference mirror oscillation, allowing for a comparative analysis with the conventional SOCT method. All human whole blood samples were prepared, using the blood of six anonymized, healthy donors from the Experimental Centre for Technical Medicine (ECTM) of the University of Twente. All methods were carried out in accordance with 
relevant guidelines and regulations. In agreement with the Declaration of Helsinki, informed consent was obtained from all volunteers and the used blood collection procedure was approved by the local Medical Research Ethics Committee (METC Twente). The [tHb] of the samples was varied by diluting the blood with PBS, as well as thickening the blood by removing plasma. Visual inspection of the plasma showed no signs of haemolysis in all samples. All blood samples were exposed to air during preparation and contained Heparin as anticoagulant. Expected [tHb] values for all samples were obtained by reference measurements using a blood analyser (Avoximeter 1000E, Instrumentation Laboratory, USA). Both the SOCT and reference measurements were performed in triplo. One focus tracking and zero-delay acquisition and one conventional sOCT measurement (expected $[\mathrm{tHb}]=19.4 \mathrm{~g} / \mathrm{dL}$ ) were excluded from this study after visual inspection of the A-scans, which revealed that no scattering medium (i.e. blood) was present inside the measurement volume - most likely to be caused by an obstruction inside the capillary. As a result, these data points were measured only in duplo. For the conventional SOCT method, two samples with high haemoglobin concentrations were excluded, as no useable attenuation spectra could be obtained due to excessive signal loss (expected [tHb] of 19.1 $\mathrm{g} / \mathrm{dL}$ and $21.5 \mathrm{~g} / \mathrm{dL}$ ). For one other sample in the conventional sOCT analysis, two out of three measurements (expected $[\mathrm{tHb}]=9.10 \mathrm{~g} / \mathrm{dL}$ ) had to be excluded due to an unforeseen interference of the complex conjugate with the signal, leaving a single measurement for this data point.

\section{Bibliography}

1 World Health Organization. "Haemoglobin concentrations for the diagnosis of anaemia and assessment of severity," at https://www.who.int/vmnis/indicators/haemoglobin/en/ (2011).

2 Jeon, K. J., Kim, S. \& Park, K. K. "Noninvasive total hemoglobin measurement," J. Biomed. Opt. 7(1), 45-50 (2002).

3 Crighton, G. L., New, H. V., Liley, H. G. \& Stanworth, S. J. "Patient blood management, what does this actually mean for neonates and infants?" Transfus. Med. 28(2), 117-131 (2018).

4 Shamir, M. Y., Avramovich, A. \& Smaka, T. "The current status of continuous noninvasive measurement of total, carboxy, and methemoglobin concentration," Anesth. Analg. 114(5), 972-978 (2012).

5 Saerchen, E. et al. "Focused, wide-band, polymer-based optoacoustic transducers for noninvasive monitoring of total hemoglobin 
concentration and other blood variables," Proc. SPIE 7899, Photons Plus Ultrasound: Imaging and Sensing. 78994E.

6 Bosschaart, N., Faber, D. J., Leeuwen, T. G. \& Aalders, M. C. "In vivo lowcoherence spectroscopic measurements of local hemoglobin absorption spectra in human skin," J. Biomed. Opt. 16(10), 100504 (2011).

$7 \quad \mathrm{Pi}$, S. et al. "Automated spectroscopic retinal oximetry with visible-light optical coherence tomography," Biomed. Opt. Express 9(5), 2056-2067 (2018).

8 Chong, S. P., Merkle, C. W., Leahy, C., Radhakrishnan, H. \& Srinivasan, V. J. "Quantitative microvascular hemoglobin mapping using visible light spectroscopic Optical Coherence Tomography," Biomed. Opt. Express 6(4), 1429-1450 (2015).

$9 \quad \mathrm{Yi}, \mathrm{J}$. \& Li, X. "Estimation of oxygen saturation from erythrocytes by high resolution spectroscopic optical coherence tomography," Opt. Lett. 35(12), 2094-2096 (2010).

$10 \mathrm{Yi}$, J., Chen, S., Backman, V. \& Zhang, H. F. "In vivo functional microangiography by visible-light optical coherence tomography," Biomed. Opt. Express 5(10), 3603-3612 (2014).

11 Chen, S., Yi, J. \& Zhang, H. F. "Measuring oxygen saturation in retinal and choroidal circulations in rats using visible light optical coherence tomography angiography," Biomed. Opt. Express 6(8), 2840-2853 (2015).

$12 \mathrm{Yi}$, J. et al. "Visible light optical coherence tomography measures retinal oxygen metabolic response to systemic oxygenation," Light Sci. Appl. 4, e334 (2015).

13 Veenstra, C., Petersen, W., Vellekoop, I. M., Steenbergen, W. \& Bosschaart, N. "Spatially confined quantification of bilirubin concentrations by spectroscopic visible-light optical coherence tomography," Biomed. Opt. Express 9(8), 3581-3589 (2018).

14 Robles, F. E., Chowdhury, S. \& Wax, A. "Assessing hemoglobin concentration using spectroscopic optical coherence tomography for feasibility of tissue diagnostics," Biomed. Opt. Express 1(1), 310-317 (2010).

15 Vásquez, R. \& Villena, M. "Normal hematological values for healthy persons living at 4000 meters in Bolivia," High alt. med. biol. 2(3), 361367 (2001). 
16 Bosschaart, N., Aalders, M. C. G., van Leeuwen, T. G. \& Faber, D. J. "Spectral domain detection in low-coherence spectroscopy," Biomed Opt Express 3(9), 2263-2272 (2012).

17 Almasian, M., Bosschaart, N., van Leeuwen, T. G. \& Faber, D. J. "Validation of quantitative attenuation and backscattering coefficient measurements by optical coherence tomography in the concentrationdependent and multiple scattering regime," J. Biomed. Opt. 20(12), 121314 (2015).

18 Bosschaart, N., Edelman, G. J., Aalders, M. C., van Leeuwen, T. G. \& Faber, D. J. "A literature review and novel theoretical approach on the optical properties of whole blood," Lasers Med. Sci. 29(2), 453-479 (2014).

19 Bosschaart, N. et al. "Quantitative measurements of absorption spectra in scattering media by low-coherence spectroscopy," Opt. Lett. 34(23), 3746-3748 (2009).

20 Baskurt, O. K., Hardeman, M. R. \& Rampling, M. W. "Handbook of Hemorheology and Hemodynamics," (IOS Press, 2007).

21 Weiss, N., van Leeuwen, T. G. \& Kalkman, J. "Localized measurement of longitudinal and transverse flow velocities in colloidal suspensions using optical coherence tomography," Phys. Rev. E 88(4), 042312 (2013).

22 Yun, S. H., Tearney, G. J., Bouma, B. E., Park, B. H. \& de Boer, J. F. “Highspeed spectral-domain optical coherence tomography at $1.3 \mu \mathrm{m}$ wavelength," Opt Express 11(26), 3598-3604 (2003).

23 Duyens, L. N. M. "The flattering of the absorption spectrum of suspensions, as compared to that of solutions," Biochim. Biophys. Acta 19, 1-12 (1956). 


\title{
Chapter 4:
}

\section{Optical properties of human milk}

\begin{abstract}
With human milk being the most important source of infant nutrition, the protection and support of breastfeeding are essential from a global health perspective. Nevertheless, relatively few objective methods are available to investigate human milk composition and lactation physiology when a mother experiences breastfeeding problems. We argue that optics and photonics offer promising opportunities for this purpose. Any research activity within this new application field starts with a thorough understanding on how light interacts with human milk. Therefore, the aim of this study was to investigate the full set of optical properties for human milk and the biological variability therein. Using a novel approach that combines spatially resolved diffuse reflectance spectroscopy (SR-DRS) and spectroscopic optical coherence tomography (sOCT) between $450-650 \mathrm{~nm}$, we quantified the absorption coefficient $\mu_{a}$, scattering coefficient $\mu_{s}$, reduced scattering coefficient $\mu_{s}^{\prime}$, anisotropy $g$ and backscattering coefficient $\mu_{b, N A}$ of mature human milk from 14 participants released at different stages during a breastfeed (foremilk, bulk milk and hindmilk). Significant correlations were found between $\mu_{a}, \mu_{s}, \mu_{s}{ }^{\prime}$ and $\mu_{b, N A}$ and the biochemically determined fat concentration per sample $\left(R_{s}=0.38, R_{s}=0.77\right.$, $R_{s}=0.80, R_{s}=0.44$ respectively). We explained the observed variations in the optical properties of human milk using Mie theory and the biological variability in both the concentration and size distribution of milk fat globules. In conclusion, we have provided a full set of optical properties for human milk, which can hopefully serve as a starting point for future biophotonic studies on human milk and the milk containing lactating breast.
\end{abstract}

This chapter is published as: Colin Veenstra, Anki Lenferink, Wilma Petersen, Wiendelt Steenbergen, and Nienke Bosschaart, "Optical properties of human milk," Biomed. Opt. Express 10(8), 4059-4074 (2019) 


\subsection{Introduction}

From a medical, social, economic and environmental perspective, human milk is the optimal source of nutrition for infants in early life [1,2]. Millions of years of evolution have adapted human milk composition to optimally support infant survival, growth, health and cognitive development [3]. Abundant evidence demonstrates that breastfeeding protects infants against many types of infections, diarrhea and dental malocclusions, and increases intelligence [1]. Also mothers benefit from breastfeeding, since it reduces the risk for breast cancer and improves birth spacing [1]. As a consequence, the deaths of 823,000 children and 20,000 mothers can be averted annually if all mothers worldwide would breastfeed their infants [1].

But despite all of this evidence, worldwide breastfeeding rates do not comply with the advice of the World Health Organization for mothers to exclusively breastfeed their infants until the age of 6 months, followed by continued breastfeeding with complimentary foods until a minimum age of 2 years [4]. In many high-income countries, breastfeeding rates at the age of 1 year are less than $20 \%$, with less than $1 \%$ in the United Kingdom. The most important reasons for mothers to abandon breastfeeding are the perception of insufficient milk supply (PIM) and pain due to breastfeeding problems [5]. Besides many political and cultural challenges, solving this issue also involves a crucial responsibility for scientific research. Compared to other fields of medical research, the scientific activity on human milk and lactation research is underrepresented [6]. Nevertheless, for those mothers who experience breastfeeding problems, lactation support will benefit from a better scientific understanding on lactation physiology and pathology, as well as human milk composition and function.

Photonics offers many promising opportunities for the development of objective methods for the investigation of human milk and lactation, which to date have remained largely unexplored. Technologies like near-infrared spectroscopy (NIRS) [7], laser Doppler perfusion monitoring (LDPM) [8,9], diffuse optical imaging (DOI) [10] and photoacoustic mammography (PAM) can potentially provide important new insights into the dynamics of mammary tissue composition and the hemodynamics of lactation. In turn, these factors can be related to milk synthesis, milk removal from the breast and the development of breastfeeding problems, such as mastitis. Regarding human milk analysis, chemically highly specific optical technologies such as Raman 
spectroscopy have only been marginally explored for this purpose [11-14]. Human milk macronutrient analysis with NIRS is commercially available, but the technology needs to be improved for its main purpose of personalized human milk fortification in premature infants [15]. Therefore, it leaves no doubt that the biophotonics research community has a valuable opportunity to explore this important new area of application.

Comparable to any other application field in biophotonics, the optical investigation of both human milk and the milk containing lactating breast starts with a thorough understanding of how light interacts with human milk itself, which requires knowledge on its currently unexplored optical properties. These optical properties are essential for the prediction, modelling and interpretation of light tissue interactions. From studies on bovine milk, we know that the predominant scattering particles in milk are the fat globules (up to around 11 $\mu \mathrm{m}$ in diameter), followed by the casein micelles (around $200 \mathrm{~nm}$ in diameter) $[16,17]$. It is expected that a large variation exists within the normal range of optical properties of human milk, since human milk varies considerably in fat content between mothers, within individual mothers over the course of lactation, between consecutive feedings, and even within a single breastfeed [18]. The latter is caused by the fact that the milk released at the onset of a breastfeed (foremilk) generally has a lower fat concentration than the milk released at the end of a breastfeed (hindmilk). As a consequence, fat concentrations in human milk have been reported to vary between approximately 5 and $100 \mathrm{~g} / \mathrm{kg}[15,18]$.

The aim of this study is therefore to provide a full set of the optical properties of human milk. Using a novel combination of spectroscopic optical coherence tomography (sOCT) and spatially resolved diffuse reflectance spectroscopy (SRDRS), we quantify the absorption coefficient $\mu_{a}$, the scattering coefficient $\mu_{s}$, backscattering coefficient $\mu_{b, N A}$, reduced scattering coefficient $\mu_{s}{ }^{\prime}$ and scattering anisotropy $g$ in the visible wavelength range $(450-650 \mathrm{~nm})$ of the foremilk, bulk milk and hindmilk from 14 volunteers. Since fat globules are the predominant scattering particles in human milk, we explain the biological variation in optical properties between milk samples using Mie theory, the biochemically determined fat concentration, and the fat globule size distribution per sample. 


\subsection{Materials and methods}

\subsubsection{Participants}

The study population included healthy, breastfeeding mothers of term infants across the Netherlands. Between April and August 2018, a total of 16 women between 2 and 9 months of lactation were included (Table 4.1). The study was approved by the Committee on Research Involving Human Subjects (CMO Arnhem-Nijmegen, The Netherlands), and all participants gave written consent prior to the study.

Table 4.1: Participant information.

\begin{tabular}{cccccc}
$\begin{array}{c}\text { Partici } \\
\text { pant }\end{array}$ & $\begin{array}{c}\text { Age } \\
\text { (years) }\end{array}$ & $\begin{array}{c}\text { Gender of } \\
\text { breastfed } \\
\text { infant }\end{array}$ & $\begin{array}{c}\text { Lactation } \\
\text { Period } \\
\text { (Months) }\end{array}$ & $\begin{array}{c}\text { Time since last } \\
\text { breastfeed } \\
\text { (hours) }\end{array}$ & $\begin{array}{c}\text { Breast for sample } \\
\text { collection }\end{array}$ \\
\hline $\mathbf{1}$ & 35 & M & 8.5 & 8 & Unknown \\
\hline $\mathbf{2}^{\text {a }}$ & 35 & M & 8.5 & 7 & Both \\
$\mathbf{3}^{\text {a }}$ & 31 & M & 2 & 4 & Right \\
$\mathbf{4}$ & 32 & M & 4 & 7 & Right \\
\hline $\mathbf{5}$ & 35 & M & 3 & 3 & Left \\
$\mathbf{6}$ & 26 & F & 7.5 & 6.5 & Right \\
\hline $\mathbf{7}$ & 31 & M & 4 & 4 & Left \\
$\mathbf{8}$ & 31 & M & 3.5 & 4 & Left \\
\hline $\mathbf{9}$ & 26 & F & 4 & 4 & Right \\
\hline $\mathbf{1 0}$ & 32 & F & 5.5 & 8 & Right \\
\hline $\mathbf{1 1}$ & 32 & M & 2.5 & 11 & Unknown \\
\hline $\mathbf{1 2}$ & 31 & M & 2.5 & 12 & Right \\
\hline $\mathbf{1 3}$ & 32 & F & 4 & 4 & Right \\
\hline $\mathbf{1 4}$ & 34 & M & 3.5 & 4 & Right \\
\hline $\mathbf{1 5}$ & 34 & M & 2 & 3 & Left \\
\hline $\mathbf{1 6}$ & 27 & M & 3.5 & 3 & Right \\
\hline
\end{tabular}

${ }^{a}$ Excluded participants from this study, due to deviation from the sample collection procedure.

\subsubsection{Acquisition of human milk samples}

All participants followed a step-wise protocol for milk extraction, during which they collected $2 \times 1 \mathrm{~mL}$ of foremilk, $2 \times 1 \mathrm{~mL}$ of bulk milk and $2 \times 1 \mathrm{~mL}$ of hindmilk. The $1 \mathrm{~mL}$ milk samples were stored in two separate containers: one for the 
optical property measurements and one for the biochemical determination of fat concentration. The participants used their own breast pump for single-side milk expression on the breast that had not been emptied for the longest period of time. Most participants collected the samples autonomously, but optional support was provided by a researcher upon request of the participant herself.

The protocol involved the following steps: 1) after expression of the first $5 \mathrm{~mL}$, the participant interrupted the milk expression to collect $2 \times 1 \mathrm{~mL}$ of the foremilk, 2) milk expression was resumed until the breast had almost been emptied completely, after which milk expression was interrupted again, and 3) the last $5 \mathrm{~mL}$ were collected in a separate milk container, from which the participant collected $2 \times 1 \mathrm{~mL}$ of hindmilk. The $2 \times 1 \mathrm{~mL}$ of bulk milk was collected from the total expressed milk volume. After cooled transport, the collected samples were stored at $-18{ }^{\circ} \mathrm{C}$ for a maximum period of 10 months, until the measurements were performed. The milk samples from two participants (\#2 and \#3) were excluded from this study, due to a deviation from the sample collection procedure. Therefore, this study included milk samples from 14 participants, leading to a total of 42 milk samples for optical property determinations.

\subsubsection{Sample preparation for optical property measurements}

After thawing the milk samples in a water bath at $20^{\circ} \mathrm{C}$, the samples were homogenized by inverting them by hand, followed by pipetting the entire sample volume up and down several times. Automated homogenization methods such as sonication or vortexing were not used, as these may change the size of the fat globules, which can affect the optical properties $[15,19]$.

High fat concentrations can hinder successful determination of the optical properties, either due to the contribution of multiple scattering to the SOCT signal $[20,21]$, or due to high signal losses in the diffuse reflectance setup. Therefore, strongly scattering milk samples were diluted with phosphate buffered saline (PBS) until $\mu_{s}<6 \mathrm{~mm}^{-1}$ or $\mu_{s}{ }^{\prime}<1 \mathrm{~mm}^{-1}$ at $550 \mathrm{~nm}-$ i.e. values for which the measured optical properties scaled linear with the dilution factor. Assuming concentration independence for single scattering events, the optical properties of the original sample were retrieved by rescaling the measured optical properties with the dilution factor. All optical property measurements were performed in triplo. 


\subsubsection{Determination of fat concentration}

A modified Mojonnier ether extraction method for human milk sample volumes of around $1 \mathrm{~mL}$ was used to determine the fat concentration of all samples [22]. In short, the total mass of the milk sample was registered, followed by dissolving the milk fat globules in ether. After centrifuging, a fat containing ether layer was formed. Isolation of this layer, followed by evaporation of the ether allowed for measurements of the mass of the remaining fat. As a result, the fat concentration was expressed in grams of fat per kilogram milk. The coefficient of variation for this procedure was determined by the in triplo measurement on an extra bulk milk sample ( $>3 \mathrm{~mL}$ ) that was additionally donated by one of the participants.

\subsubsection{Spatially resolved diffuse reflectance spectroscopy (SR-DRS)}

SR-DRS is an effective method for the determination of the reduced scattering coefficient $\mu_{\mathrm{s}}^{\prime}$ and absorption coefficient $\mu_{a}$ [23]. We used a home-built fiberbased SR-DRS system (Figure 4.1a), in which a $400 \mu \mathrm{m}$ core diameter multimode fiber (FT400EMT, Thorlabs, Newton, NJ, USA) guided light from a halogen light source (AvaLight-Hal, Avantes, Apeldoorn, The Netherlands) to the sample. A second $400 \mu \mathrm{m}$ core diameter multimode fiber (FT400UMT, Thorlabs, Newton, $\mathrm{NJ}$, USA) guided the diffusely reflected light from the sample to a spectrometer (AvaSpec-2048, Avantes, Apeldoorn, The Netherlands). The tips of both fibers were immersed directly under the sample surface. The source-detector distance $r$ was varied by translation of the detection fiber by a motorized stage (T-LS28M, Zaber Technologies, Vancouver, Canada) from $r_{0}=2.2 \mathrm{~mm}$ to $r_{\text {end }}=4.7 \mathrm{~mm}$ in steps of $50 \mu \mathrm{m}$. The integration time was set such that the diffuse reflectance at $r_{0}$ filled approximately $80 \%$ of the dynamic range of the spectrometer. The dark noise was measured by obtaining a spectrum without sample illumination.

\subsubsection{Reduced scattering coefficient $\left(\mu_{s}^{\prime}\right)$ and absorption coefficient $\left(\mu_{a}\right)$}

The reduced scattering, and absorption coefficient were estimated by following the method of Farrell et al. [23] This diffusion theory based model describes the diffuse reflection $R_{\text {theory }}$ as a function of $r$ and the optical properties $\mu_{a}$ and $\mu_{s}$ ':

$$
R_{\text {theory }}(r)=\frac{1}{4 \pi}\left(\frac{z_{0}}{r_{1}^{2}}\left(\mu_{e f f}+\frac{1}{r_{1}}\right) e^{-\mu_{e f f} r_{1}}+\frac{z_{0}+2 z_{b}}{r_{2}^{2}}\left(\mu_{e f f}+\frac{1}{r_{2}}\right) e^{-\mu_{e f f} r_{2}}\right)
$$


With effective attenuation coefficient $\mu_{\text {eff }}=\left(3 \mu_{a}\left(\mu_{a}+\mu_{s}^{\prime}\right)\right)^{1 / 2}$, inverse total interaction coefficient $z_{0}=\left(\mu_{a}+\mu_{s}^{\prime}\right)^{-1}, r_{1}=\left(z_{0}^{2}+r^{2}\right)^{1 / 2}, r_{2}=\left(\left(z_{0}+2 z_{b}\right)^{2}\right)^{1 / 2}$, and $z_{b}=$ $(2 / 3)\left(\mu_{a}+\mu_{s}\right)^{\prime-1}$, under the conditions that $r>1 /\left(\mu_{s}{ }^{\prime}+\mu_{a}\right)$ and $\mu_{s}{ }^{\prime}>>\mu_{a}$. In the wavelength region $450-530 \mathrm{~nm}$, riboflavin, beta-carotene and fat contribute to the absorption of human milk ${ }^{14}$. Following our approach in Bosschaart, et al. [24] a more robust estimate of $\mu_{s}^{\prime}$ and $\mu_{a}$ can be obtained by a two-step fitting approach over the wavelength range with $(450-530 \mathrm{~nm})$ and without (530 $650 \mathrm{~nm}$ ) absorption, rather than a single fit of the model over the entire wavelength range.

First, by assuming negligible optical absorption between $530-650 \mathrm{~nm}$ for human milk, $\mu_{s}^{\prime}$ was retrieved in this wavelength range by fitting Eq. 4.2 to the dark noise corrected $R_{\text {meas }}(r)$ :

$$
R_{\text {meas }}(r)=\beta R_{\text {theory }}(r)
$$

with $\mu_{a}=0$ and fit parameters: scaling factor 8 and $\mu_{s}^{\prime}$. Secondly, we estimated $\mu_{s}^{\prime}$ between $450-530 \mathrm{~nm}$ by extrapolating a fitted power law to the results obtained from Eq. 4.2:

$$
\mu_{s}{ }^{\prime}=a \lambda^{-b}
$$

with fit parameters: scaling factor $a$ and scatter power $b$. Next, $\mu_{a}$ was obtained by fitting Eq. 4.2 to $R_{\text {meas }}(r)$ between $450-530 \mathrm{~nm}$, with $\mu_{s}^{\prime}$ fixed at the extrapolated values obtained from Eq. 4.3 and fit parameters $\mu_{a}$ and 6 .

In case the model validity limit $\left(r>1 /\left[\mu_{s}^{\prime}+\mu_{a}\right]\right)$ was not met, $r_{0}$ was increased until the condition is satisfied. $r_{\text {end }}$ was kept constant throughout this work.

\subsubsection{Spectroscopic optical coherence tomography (sOCT)}

SOCT is an effective method for the spectrally resolved determination of the scattering coefficient $\mu_{s}$ and backscattering coefficient $\mu_{b, N A}$, as we have demonstrated for a wide range of scattering samples $\left(\mu_{s}=0.15-34 \mathrm{~mm}^{-1}, \mu_{b, N A}=\right.$ $2 \cdot 10^{-6}-2 \cdot 10^{-3} \mathrm{~mm}^{-1}$ ) in our previous work $[20,25]$. We used a broadband Michelson interferometer-based SOCT system, which measured the lowcoherent interference between the backscattered light from the sample and a reference beam (Figure 4.1b). Short time Fourier transformation with a spectral resolution of $5 \mathrm{~nm}$ resulted in a spatially and spectrally confined dataset of the 
backscattered intensity from the sample $S(\lambda, d)$ with a spatial resolution ranging from $15 \mu \mathrm{m}$ (at $\lambda=450 \mathrm{~nm}$ ) to $31 \mu \mathrm{m}$ (at $\lambda=650 \mathrm{~nm}$ ). The lateral resolution of the system was $2.5 \mu \mathrm{m}$ in air. Our system combined focus tracking with zerodelay acquisition, which ensures that the measured attenuation of $S(\lambda, d)$ as a function of sample depth $d$ is only affected by the optical attenuation of the sample itself. A detailed description of our SOCT system is given in Veenstra, et al. [26]. In short, focus tracking was achieved by translation of the sample lens with respect to the sample. Zero-delay acquisition at all investigated depths within the sample is was achieved by filtering out the DC component and mirror image from the Doppler shifted signal, by an oscillating reference mirror.

\subsubsection{Scattering coefficient $\left(\mu_{s}\right)$}

Under the assumption of single scattering, the sample's attenuation spectrum $\left(\mu_{t}=\mu_{s}+\mu_{a}\right)$ can be quantified by fitting Beer's law to $S(\lambda, d)[20]$ :

$$
\ln \left(\left(S(\lambda, d)-S_{b g}\right)^{2}\right)=\ln (\alpha(\lambda))-2 \mu_{t}(\lambda) d
$$

with fit parameters $\alpha$ and $\mu_{t}$. The background term $S_{b g}$ was obtained from a measurement at a depth of $1 \mathrm{~mm}$ inside the cuvette, where the signal had been attenuated completely. Measurements were performed over a depth interval $d$ $=150-500 \mu \mathrm{m}$ relative to the sample's surface. The scattering coefficient was obtained by correcting $\mu_{t}$ for the previously determined $\mu_{a}$ from the SR-DRS measurement (Figure 4.1c): $\mu_{s}=\mu_{t}-\mu_{a}$.

\subsubsection{Backscattering coefficient $\left(\mu_{b, N A}\right)$}

The backscattering coefficient of the sample within the NA $=0.08$ of the sOCT system can be obtained from $\alpha(\lambda)$ in eq. 4. Hereto, it is assumed that $\alpha(\lambda)$ consists of two factors [20]:

$$
\alpha(\lambda)=\mu_{b, N A}(\lambda) \zeta(\lambda)
$$

with backscattering coefficient $\mu_{\mathrm{b}, N \mathrm{~A}}$ and system efficiency term $\zeta(\lambda)$, which includes system dependent parameters such as illumination and coupling efficiency. $\zeta(\lambda)$ was estimated by a calibration measurement on a suspension of NIST-certified polystyrene spheres $(2 \mathrm{mg} / \mathrm{mL}$, diameter $400 \mathrm{~nm})$, from which the 
$\mu_{\mathrm{b}, \mathrm{NA}}$ was exactly known from Mie theory [20]. Subsequently, $\mu_{b, N A}$ was retrieved from $\alpha(\lambda)$ by $\mu_{b, N A}(\lambda)=\alpha(\lambda) / \zeta(\lambda)$.

\subsubsection{Anisotropy (q)}

The anisotropy factor $g$ was obtained indirectly by combining the results from $\mu_{s}$ and $\mu_{s}^{\prime}$ (Figure 4.1c), following:

$$
g=1-\frac{\mu_{s}{ }^{\prime}}{\mu_{s}}
$$

\subsubsection{Mie theory}

Besides the experimentally derived optical properties, we used Mie-theory calculations in MatScat $[27,28]$ to investigate the influence of the fat globule size distribution, as well as the less dominant scattering contribution by casein on the optical scattering properties of human milk. Hereto, milk was modeled as a suspension of scattering casein micelles and fat globules in whey with refractive index $n_{\text {whey }}=1.345$ [29]. The concentration of casein micelles $N_{\text {cas }}$ and their scattering cross section $\sigma_{\text {cas }}$ were approached by modelling casein micelles as monodisperse homogeneous spheres with the average properties of bovine casein micelles $[16,17,30]$ : diameter $D_{\text {casein }}=200 \mathrm{~nm}$ and $n_{\text {casein }}=1.5$, as literature values for human milk casein micelles were unavailable. The concentration of casein micelles was fixed to the average concentration for human milk (1.68 $\mathrm{mL} / \mathrm{L}$ [31]), since casein concentrations do not vary significantly between foremilk, bulk milk and hindmilk [18]. To account for the high amount of variation in fat globule scattering between milk samples, we used the sample dependent fat globule size distribution as an input for our Mie calculations. All Mie calculations were performed at $550 \mathrm{~nm}$.

\subsubsection{Fat globule size distribution}

Fat globule size distributions with $0.5 \mu \mathrm{m}$ bins were obtained from 3 bright field microscopic images per sample (EVOS FL Cell Imaging System, Thermo Fisher, MA, USA). Images were processed by Matlab (2015b, Mathworks, MA, USA) using the built-in function 'imfindcircles' to retrieve particle diameters. To investigate the variation in fat globule size distributions between participants and milk samples, the centroid of the size distribution was calculated for every sample. The average size distributions for foremilk, bulk milk and hindmilk were 
used as an input for the Mie-calculations. To evaluate the variation in optical properties due to variations in size distribution, Mie-calculations were also performed for the size distributions of the individual samples.

Furthermore, we investigated the relation between fat globule size and the scattering properties of human milk. Hereto, we first normalized the scattering properties of all individual samples with their fat concentration, ruling out the influence of fat concentration on the scattering properties. Subsequently, we calculated the correlation between the normalized scattering properties with the centroid diameter.

\subsubsection{Mie calculations of optical properties}

Fat globules were modeled as homogeneous spheres $[32,33]$ with $n_{\text {fat }}=1.46$ and Mie calculations were performed independently for every fat globule diameter $i$ in both the individual, and the average fat globule size distribution per milk type, resulting in scattering cross section $\sigma_{s, f a t, i}$ and phase function $P(\theta)_{f a t, i .}$ The amount of fat globules with diameter $i$ per volume $N_{f a t, i}$ was calculated using densities of $0.92 \mathrm{~g} / \mathrm{ml}$ [34] and $1.03 \mathrm{~g} / \mathrm{ml}$ [35] for fat and milk, respectively. The scattering coefficient of each milk sample was calculated by a summation of the individual contributions of all scattering particles:

$$
\mu_{s, \text { Mie }}=\sum_{i} N_{f a t, i} \sigma_{s, f a t, i}+N_{c a s} \sigma_{s, c a s}
$$

The individual (unnormalized) phase functions of all particles were summed to acquire a combined phase function:

$$
P(\theta)_{\text {com }}=\frac{\sum_{i}\left(N_{f a t, i} P(\theta)_{f a t, i}\right)+N_{c a s} P(\theta)_{c a s}}{N_{\text {total }}}
$$

Followed by calculation of the anisotropy from the combined phase function:

$$
g_{\text {Mie }}=\frac{2 \pi \int_{0}^{\pi} P(\theta)_{c o m} \cos \theta \sin \theta d \theta}{2 \pi \int_{0}^{\pi} P(\theta)_{c o m} \sin \theta d \theta}
$$


The reduced scattering coefficient was obtained by combining the results for $\mu_{s}$ and $g$ :

$$
\mu_{s \text { Mie }}{ }^{\prime}=\mu_{s, \text { Mie }}\left(1-g_{\text {Mie }}\right)
$$

Similar to the scattering coefficient, the backscattering coefficient was calculated by summation of the individual contributions of all particles, followed by integration over the solid angle of the NA in the medium [20]:

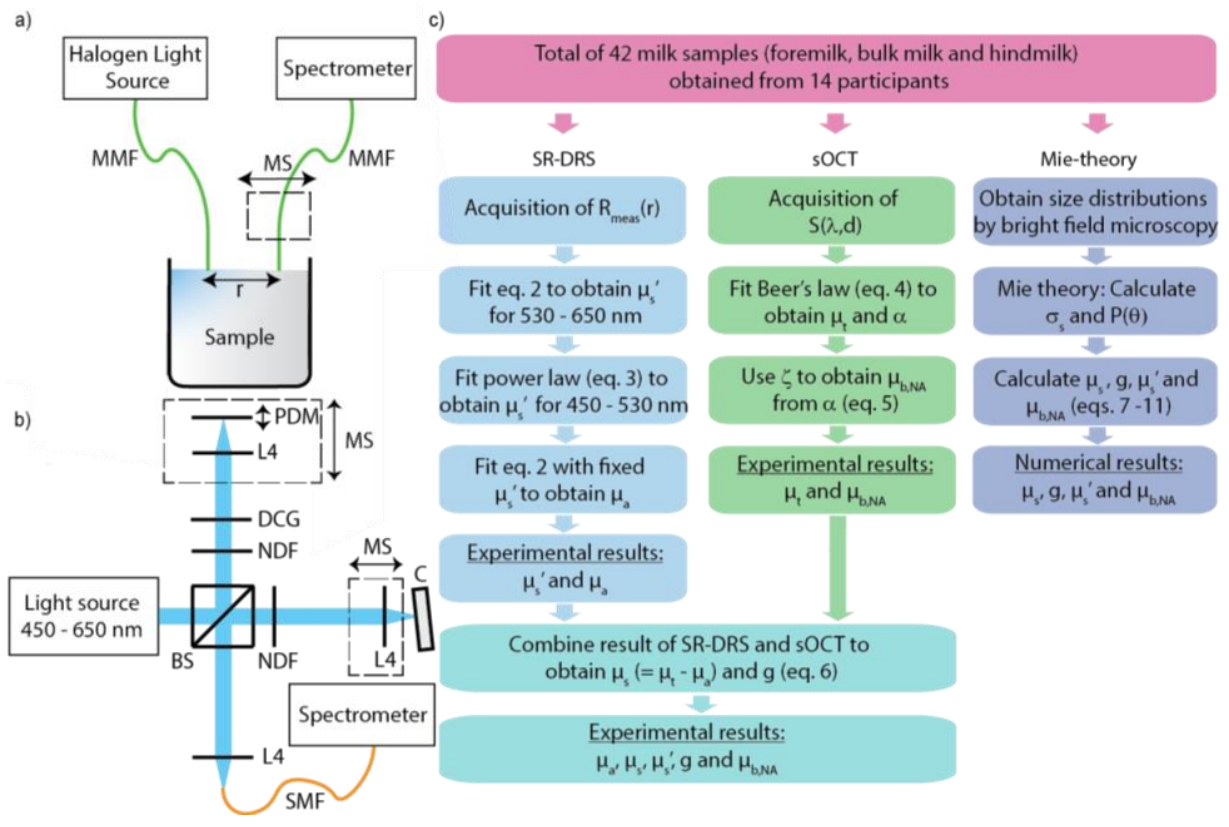

Figure 4.1: Schematic overview of the optical methods used to determine the full set of optical properties. a) Illustration of the SR-DRS setup. Light from the illumination fiber diffuses through the sample, which can be detected as a function of inter-fiber distance $r$ by translation of the detection fiber. b) Schematic overview of the SOCT setup. c) Flowchart of the experimental and numerical methods in this study, with the optical properties they provide. MMF: multi mode fiber, $r$ : source-detector distance, MS: motorized stage, NDF: neutral density filters, L\#: lens \#, BE: beam expander, SPF: short pass filter, M: mirror, $B S$ : beam splitter, DCG: dispersion compensation glass, PDM: piezo driven mirror, C: cuvette with sample, SMF: single mode fiber. 


$$
\mu_{b, N A, M i e}=\sum_{i}\left(N_{f a t, i} \sigma_{s, f a t, i} \frac{2 \pi \int_{\pi-N A}^{\pi} P(\theta)_{f a t, i} \sin (\theta) d \theta}{2 \pi \int_{0}^{\pi} P(\theta)_{f a t, i} \sin (\theta) d \theta}\right)+N_{c a s} \sigma_{s, c a s} \frac{2 \pi \int_{\pi-N A}^{\pi} P(\theta)_{c a s} \sin (\theta) d \theta}{2 \pi \int_{0}^{\pi} P(\theta)_{c a s} \sin (\theta) d \theta}
$$

\subsection{Results}

\subsubsection{Experimentally derived optical property spectra}

The median spectra and their range of variation for all experimentally derived optical properties are shown in Figure 4.2 for foremilk, bulk milk and hindmilk. The absorption coefficient shows a peak around $460 \mathrm{~nm}$, which can be ascribed to absorption by riboflavin or beta-carotene. Values of $\mu_{a}$ at $460 \mathrm{~nm}\left(\mu_{a, 460 \mathrm{~nm}}\right)$ range between $1.1 \times 10^{-2}$ to $8.3 \times 10^{-2} \mathrm{~mm}^{-1}$. The absorption shows an increasing trend with milk type: from foremilk, to bulk milk and hindmilk, the median $\mu_{a, 460 \mathrm{~nm}}$ increases from $1.7 \times 10^{-2} \mathrm{~mm}^{-1}$, to $2.6 \times 10^{-2} \mathrm{~mm}^{-1}$ and $4.7 \times 10^{-2} \mathrm{~mm}^{-1}$.

Also the measured scattering properties $\mu_{s}, \mu_{s}^{\prime}$ and $\mu_{b, N A}$ vary considerably between samples, with a total range at $550 \mathrm{~nm}$ in $\mu_{s, 550 \mathrm{~nm}}=2.6-21.0 \mathrm{~mm}^{-1}$, $\mu_{s}{ }^{\prime}, 550 \mathrm{~nm}=0.24-2.48 \mathrm{~mm}^{-1}$, and $\mu_{b, N A, 550 \mathrm{~nm}}=1.1 \times 10^{-4}-1.6 \times 10^{-3} \mathrm{~mm}^{-1}$. Overall, the value of scattering properties $\mu_{s}, \mu_{s}{ }^{\prime}$ and $\mu_{b, N A}$ increase with milk type: from foremilk, to bulk milk and hindmilk, the median $\mu_{s, 550 \mathrm{~nm}}$ increases from $4.9 \mathrm{~mm}^{-}$ 1 , to $9.7 \mathrm{~mm}^{-1}$ and $15.7 \mathrm{~mm}^{-1}$, the median $\mu_{s}{ }^{\prime}, 550 \mathrm{~nm}$ increases from $0.44 \mathrm{~mm}^{-1}$, to $0.79 \mathrm{~mm}^{-1}$ and $1.58 \mathrm{~mm}^{-1}$, and the $\mu_{b, N A, 550 \mathrm{~nm}}$ varies from $4.1 \times 10^{-4} \mathrm{~mm}^{-1}$, to 4.0 $\times 10^{-4} \mathrm{~mm}^{-1}$ and $4.5 \times 10^{-4} \mathrm{~mm}^{-1}$. Large variations can be observed within milk types (foremilk, bulk milk and hindmilk), with differences between the lowest

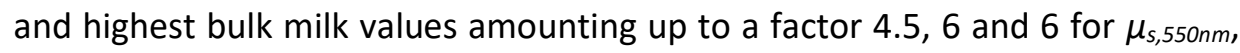
$\mu_{s}{ }^{\prime}, 550 \mathrm{~nm}$, and $\mu_{b, N A, 550 \mathrm{~nm}}$, respectively. The measured $\mu_{s}$ spectra have a curved shape with an increase in $\mu_{s}$ beyond $600 \mathrm{~nm}$.

The scattering anisotropy $g_{550 n m}$ ranges from 0.82 to 0.96 . Results are similar for the three milk types with median $g_{550 \mathrm{~nm}}$ values of $0.91,0.92$ and 0.90 for respectively foremilk, bulk milk and hindmilk. Foremilk shows less variation in anisotropy between participants, compared to bulk milk, and hindmilk. 


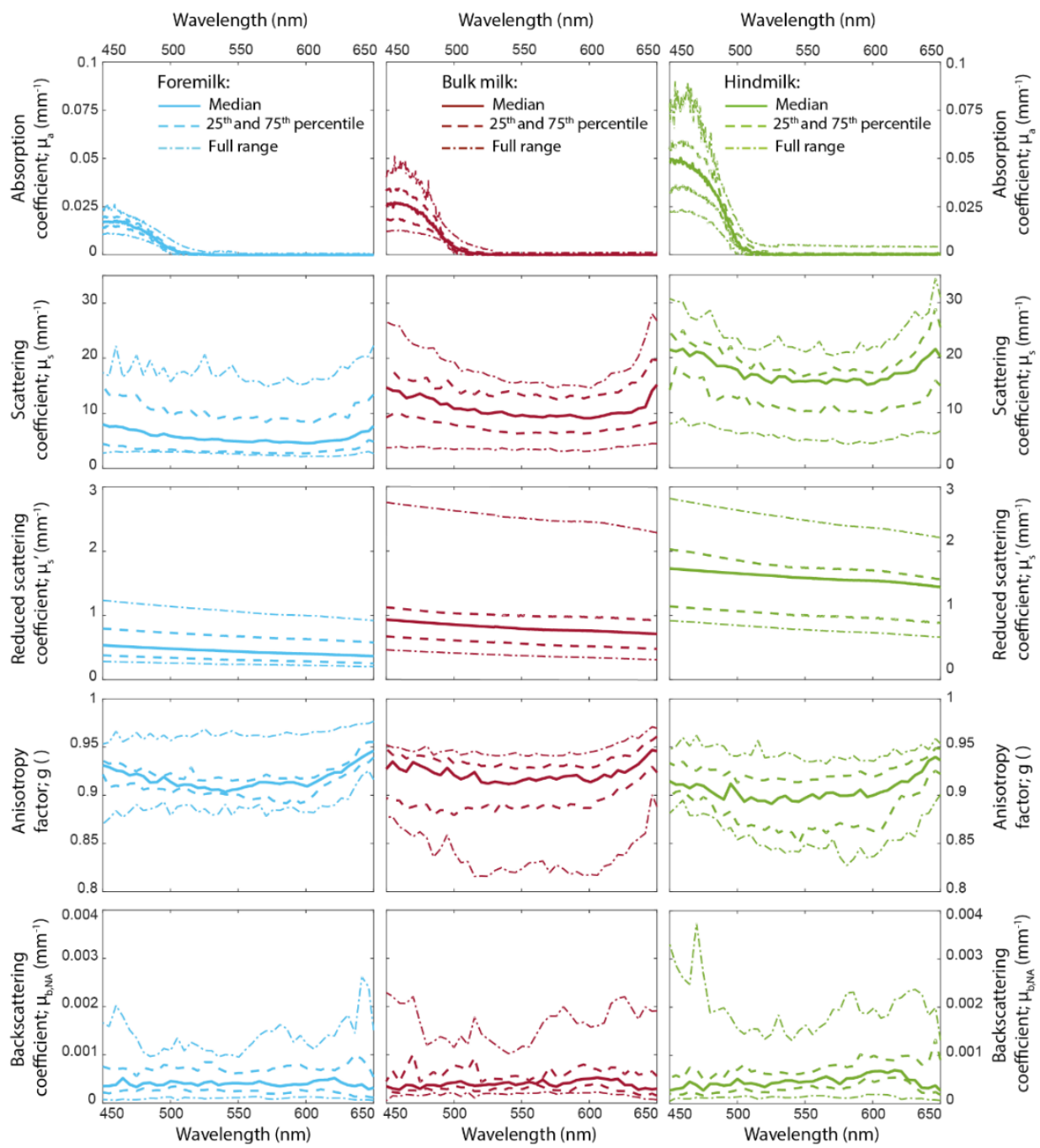

Figure 4.2: Experimentally derived optical property spectra for foremilk, bulk milk and hindmilk. Thick, solid lines represent the median values for all participants, dashed lines represent the $25^{\text {th }}$ and $75^{\text {th }}$ percentiles, and dash-dot lines represent the minimum and maximum values $\left(0^{\text {th }}\right.$ and $100^{\text {th }}$ percentile).

\subsubsection{Fat globule size distributions}

Figure 4.3a shows the average fat globule size distributions per milk type, normalized to the sum of fat globules per milk type. The average size distributions of foremilk, bulk milk and hindmilk all have a maximum around a 


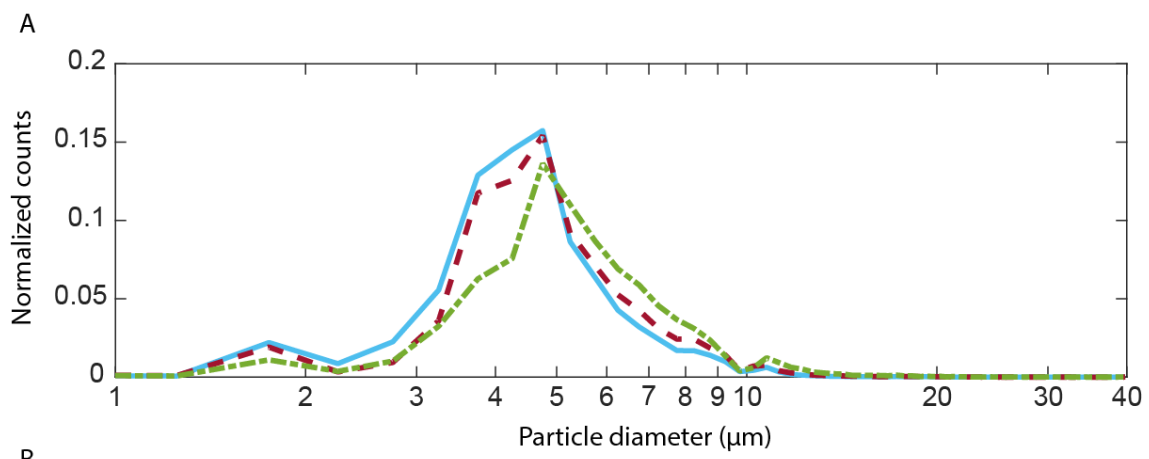

B

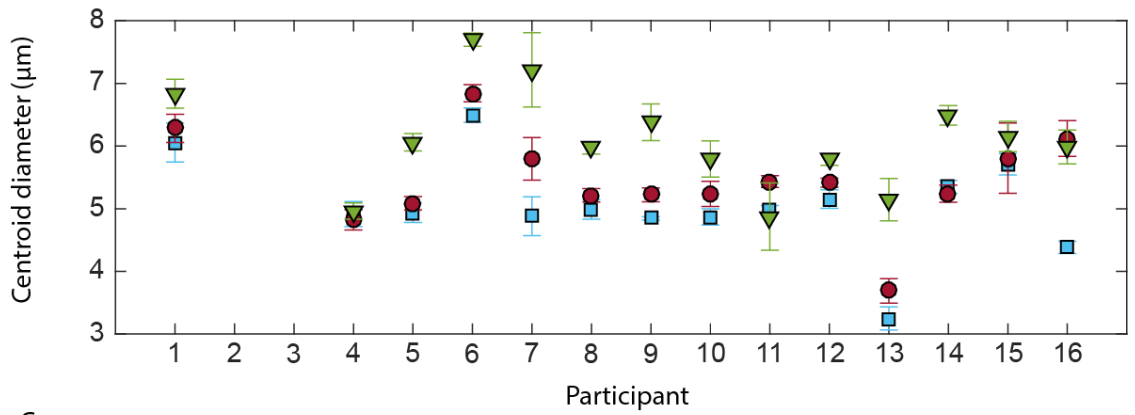

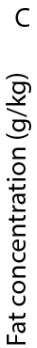

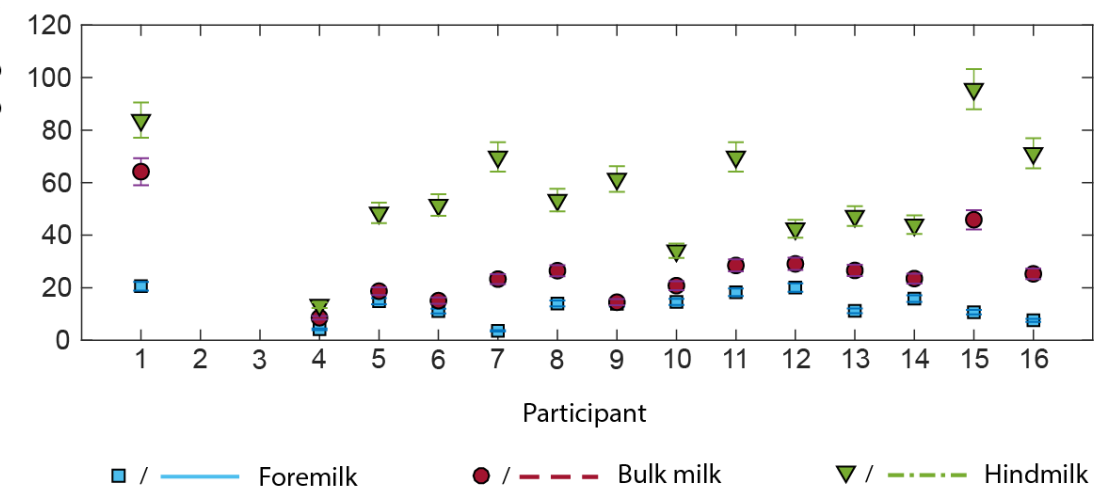

Figure 4.3: Fat globule size distributions and concentration. A: Average fat globule size distribution for foremilk, bulk milk and hindmilk, normalized with the total amount of particles. B: Centroid diameter of the fat globule size distribution for every sample within this study (error bars represent standard deviations). C: biochemically determined fat concentration per participant and sample (error bars represent the coefficient of variation in the fat determination). Participant 2 and 3 were excluded from the analysis, due to deviation from the sample collection procedure. 
particle diameter of $5 \mu \mathrm{m}$ and smaller, local maxima around particle diameters of $1.75 \mu \mathrm{m}$ and $10.5 \mu \mathrm{m}$. Figure 4.3b shows the centroid diameter for every sample per participant. For ten out of fourteen participants, foremilk has the smallest centroid diameter and hindmilk has the largest centroid diameter. The average centroid diameters are $5.2 \mu \mathrm{m}, 5.6 \mu \mathrm{m}$ and $6.2 \mu \mathrm{m}$ for foremilk, bulk milk and hindmilk respectively.

After normalizing the optical properties of all individual samples with their fat concentration, the centroid diameter of the fat globule size distribution showed weak, but significant correlation with scattering coefficient $\mu_{s}\left(R_{s}=-0.43, \mathrm{p}<\right.$ $0.01)$, anisotropy $g\left(R_{s}=-0.62, \mathrm{p}<0.01\right)$ and backscattering coefficient $\mu_{b, N A}\left(R_{s}\right.$ $=-0.44, p<0.01)$. No significant correlation was found between centroid diameter and $\mu_{s}^{\prime}\left(R_{s}=-0.20, \mathrm{p}=0.20\right)$.

\subsubsection{Optical properties versus fat concentration}

Similar to our findings on the optical properties, fat concentrations vary considerably between milk types and participants, with a range of $3.6-20.6$ $\mathrm{g} / \mathrm{kg}$ (median $14.0 \mathrm{~g} / \mathrm{kg}$ ), 8.6-64.2 g/kg (median $24.4 \mathrm{~g} / \mathrm{kg}$ ) and $13.4-95.6 \mathrm{~g} / \mathrm{kg}$ (median $52.5 \mathrm{~g} / \mathrm{kg}$ ), for foremilk, bulk milk and hindmilk, respectively. For individual participants, foremilk consistently has the lowest, and hindmilk had the highest fat concentration (Figure 4.3c). Figure 4.4 shows the measured optical properties for all samples as a function of fat concentration. The precision of our methods is indicated by the error bars, representing the standard deviation of the triplo measurements per sample. To reduce the influence of measurement noise, $\mu_{a}$ was averaged between $450-470 \mathrm{~nm}$, and all other optical properties were averaged between $530-570 \mathrm{~nm}$. The coefficient of variation for the fat determination procedure was $8 \%$, as indicated by the horizontal error bars in Figure 4.4.

The scattering properties $\mu_{s}$ and $\mu_{s}{ }^{\prime}$ increase significantly with fat concentration (Spearman correlation coefficient $R_{s}=0.77, \mathrm{p}<0.01$ and $R_{s}=0.80, \mathrm{p}<0.01$, respectively). For $\mu_{a}$ and $\mu_{b, N A}$ the correlation with fat concentration is less pronounced, but still significant $\left(R_{s}=0.38\right.$ and $R_{s}=0.44, \mathrm{p} \leq 0.01$, respectively). As expected, the scattering anisotropy $g$ does not correlate with fat concentration $\left(R_{s}=-0.02, \mathrm{p}=0.88\right)$.

The age of the mother, lactation period and time since last breastfeed showed no significant correlation with fat concentration (all $p>0.05$ ). 


\subsubsection{Mie-calculations of optical scattering properties}

The Mie-calculated optical properties based on the average fat globule size distributions of foremilk, bulk milk and hindmilk are indicated by the dashed lines in Figure 4.4. Due to the modeled scattering contribution of casein, Mie theory predicts $\mu_{s}=0.57 \mathrm{~mm}^{-1}$ and $\mu_{s}^{\prime}=0.34 \mathrm{~mm}^{-1}$ for a fat concentration of 0 $\mathrm{g} / \mathrm{kg}$. The upper and lower limits of the Mie-calculated values for the size distributions of individual samples are indicated by the dotted lines in Figure 4.4. Overall, the experimental results and Mie calculations are in the same order of magnitude and are showing similar variation between samples for both $\mu_{\mathrm{s}}$ and $\mu_{s}^{\prime}$.

Mie theory predicts that the anisotropy of casein micelles is $g_{\text {cas }}=0.41$. For high fat concentrations, the Mie-calculated $g$ approaches an asymptotic value given by the anisotropy of fat globules alone. Measured $g$ values and Mie calculations agree well for fat concentrations lower than $50 \mathrm{~g} / \mathrm{kg}$. For higher fat concentrations, Mie calculations tend to overestimate the measured $g$.

According to Mie theory, the casein contribution to the total backscattering coefficient is $\mu_{b, N A, \text { cas }}=2.3 \times 10^{-4} \mathrm{~mm}^{-1}$. On average, our Mie calculations tend to overestimate the experimentally obtained $\mu_{b, N A}$.

\subsection{Discussion}

In this study, we measured the optical property spectra $\left(\mu_{a}, \mu_{s}, \mu_{s}{ }^{\prime}, g, \mu_{b, N A}\right)$ of human milk by combining SR-DRS and SOCT, and investigated the dependency of the optical properties on fat concentration. We compared our experimental results to Mie calculations, using the fat globule size distributions of the investigated samples. The combination of SR-DRS and SOCT allowed for measurements of a full set of optical property spectra, whereas SR-DRS and SOCT individually can only provide single sets of $\mu_{a}$ and $\mu_{s}{ }^{\prime}$, or $\mu_{t}$ and $\mu_{b, N A}$, respectively. Knowledge of the full set of these optical properties will facilitate future studies that aim to investigate light tissue interactions with human milk, or the milk containing lactating breast. 

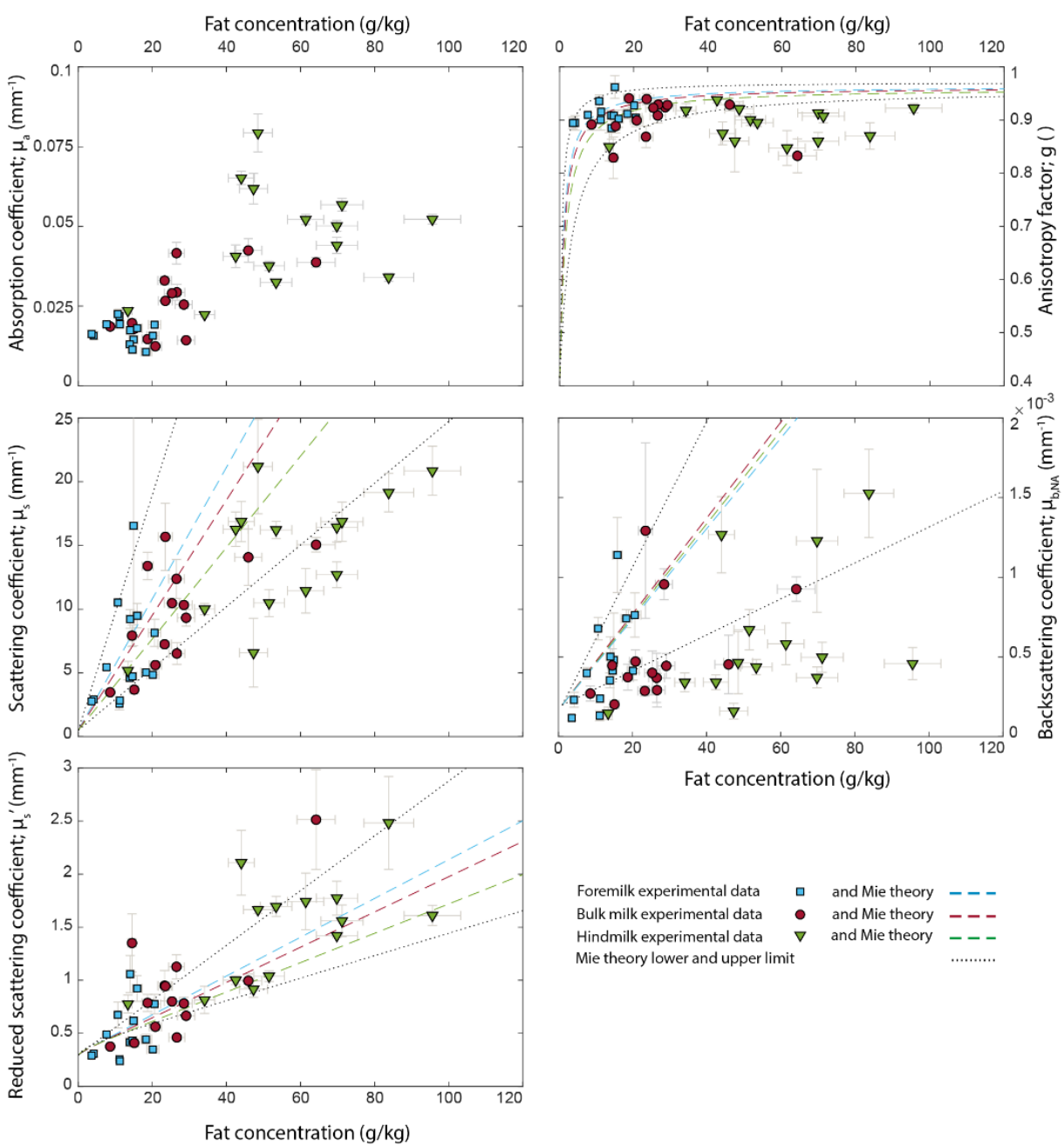
$\begin{array}{llll}\text { Foremilk experimental data } & \square & \text { and Mie theory } & --- \\ \text { Bulk milk experimental data } & - & \text { and Mie theory } & --- \\ \text { Hindmilk experimental data } & \nabla & \text { and Mie theory } & --- \\ \text { Mie theory lower and upper limit } & & \end{array}$

Figure 4.4: Optical properties versus fat concentration - experimental data and Mie calculations. The experimentally derived absorption coefficient was averaged between $\lambda=450-470 \mathrm{~nm}$, and all other experimentally derived optical properties were averaged between $\lambda=530-570 \mathrm{~nm}$. Vertical error bars represent standard deviations. Mie calculations were performed at $\lambda=550 \mathrm{~nm}$ using the average size distributions of all foremilk, bulk milk and hindmilk samples. The Mie theory upper and lower limit are based on the size distributions of individual samples. Horizontal error bars represent the coefficient of variation in the biochemical fat determination. 
Fat concentrations varied considerably between milk samples ( 3.6 to $95.6 \mathrm{~g} / \mathrm{kg}$ ). Our results are consistent with literature, where human milk fat concentrations of approximately $5-100 \mathrm{~g} / \mathrm{kg}$ have been reported [15]. Furthermore, the observed differences in fat concentrations between foremilk, and hindmilk (median values of 14.0 to $52.5 \mathrm{~g} / \mathrm{kg}$ ) are also similar to the variations reported in literature [18].

The fat globule size distributions that we obtained by bright field microscopy have a maximum at $5 \mu \mathrm{m}$ and show a smaller local maximum at $10.5 \mu \mathrm{m}$, which is in good agreement with the bulk milk size distributions that have been measured using dynamic light scattering [36,37]. To our knowledge, the differences in fat globule size distributions between foremilk, bulk milk and hindmilk have not yet been described in literature. Limited by the settings of our microscopic measurements, we were unable to identify particles smaller than $1.5 \mu \mathrm{m}$, which may explain the local maximum at $1.75 \mu \mathrm{m}$ in our fat globule size distributions. Although this neglects a considerable amount of fat globules, these smallest fat globules only account for a few percent of the milk's volume [36]. As a consequence, our Mie calculations predict that these smallest fat globules have a negligible effect $(<2 \%)$ on the scattering properties of human milk.

From the composition of human milk [31] it is expected that riboflavin and betacarotene are the main contributors to the absorption between $450-530 \mathrm{~nm}$ [38,39]. Although fat is also a chromophore in this spectral region, it contributes for only $\sim 1 \%$ to the measured absorption in the present concentrations [40]. Nevertheless, a significant correlation between the absorption coefficient and fat concentration was found, which can be explained by the high solubility of beta-carotene in fat [31].

Whereas the measured absorption coefficient of human milk is similar to that of bovine milk, the measured scattering properties (both $\mu_{s}$ and $\mu_{s}{ }^{\prime}$ ) are lower than those of bovine milk [16]. We explain this difference by higher concentrations of fat in bovine milk [41] compared to our fat estimations in human milk, as well as lower casein concentrations in human milk [31] compared to bovine milk [41]. Similar to bovine milk, scattering by human milk increases from foremilk towards hindmilk and shows a lot of variation between individuals [16]. 
As demonstrated in our previous work, the accuracy of SOCT in the determination of $\mu_{t}$ and $\mu_{b, N A}$ is approximately $10 \%$ [20], and the accuracy of SRDRS in the determination of $\mu_{\mathrm{a}}$ and $\mu_{\mathrm{s}}^{\prime}$ is approximately $15 \%$ and $10 \%$ respectively [24]. Since $\mu_{s}$ and $g$ were estimated by combining the results from both SR-DRS and SOCT, inaccuracies in either of these methods will propagate in the results for $\mu_{s}\left(=\mu_{t}-\mu_{a}\right)$ and $g\left(=1-\mu_{s}^{\prime} / \mu_{s}\right)$. However, as $\mu_{a}$ is approximately two orders of magnitude smaller than the measured $\mu_{t}$ by SOCT, any errors in the estimation of $\mu_{a}$ will be negligible in the determination of $\mu_{s}$ through $\mu_{s}=\mu_{t}$ - $\mu_{a}$. As $g$ depends on the ratio between $\mu_{s}$ and $\mu_{s}{ }^{\prime}$, the propagating error in $g$ is more substantial than for the other optical properties, amounting to an accuracy of approximately $14 \%$ with a precision of approximately $7 \%$ (error bars, Figure 4.4). Considering the spread in optical properties in both the experimental (Figure 4.2) and numerical results (Figure 4.4), the accuracy of our experimental methods was sufficient to map the full range of optical properties. The curved shape of the measured $\mu_{s}$ spectra (Figure 4.2) with an increase in $\mu_{s}$ beyond $600 \mathrm{~nm}$ can be explained by the fat globule size distributions of the milk samples, as Mie theory also predicts an increase in $\mu_{s}$ with wavelength beyond $600 \mathrm{~nm}$. Our assumption on the power law dependency of $\mu_{s}^{\prime}$ on wavelength remains valid, as Mie-theory does not predict this increase with wavelength for $\mu_{s}^{\prime}$.

To avoid the influence of multiple scattering, strongly scattering milk samples were diluted prior to the measurements. Retrieving the optical properties of the original samples by rescaling with the dilution factor can introduce an overestimation of the actual scattering properties if concentration dependent scattering occurs [21]. However, the effects of concentration dependent scattering are lower than the precision of our measurements, given the volume fraction and diameter of the major scatterers in the milk samples (fat globules of around $5 \mu \mathrm{m})[21]$.

Overall, our experimental results are described well by our Mie calculations (Figure 4.4). However, some experimental data points fall outside of the upper and lower limits of our Mie calculations. This may be caused by: I) The fixed concentration and particle size of casein for all Mie calculations. In reality, both concentration and particle size are likely to vary across samples, resulting in different outcomes for the casein contribution to the optical properties across samples. II) The assumption of scattering by homogeneous spheres in Mie 
theory. This is a simplification of fat globule and casein micelle structure, as fat globules consist of a lipid core surrounded by a phospholipid membrane [42] and casein micelles are composed of a complex of proteins and calcium phosphate [43]. III) For some of the input parameters in our Mie-calculations (casein diameter $d_{\text {casein, }}$, casein refractive index $n_{\text {casein, }}$ fat refractive index $n_{\text {fat }}$ ), we used values for bovine milk, since no literature values for human milk are available. These parameters may be different for human milk. IV) The refractive index of whey is assumed constant, while variations in whey composition may result in variations of the whey's refractive index. The refractive index for skimmed bovine milk [44] has been reported to vary between 1.345 and 1.348. Mie calculations show that this variation in refractive index may alter $\mu_{b, N A}$ up to $15 \%$, while the effect is negligible for the other scattering properties ( $<1 \%)$. In general, $\mu_{b, N A}$ estimations are very sensitive to small deviations from any of the assumptions mentioned in this paragraph, due to the fact that only $2.5 \%$ of all possible scattering angles was investigated to retrieve $\mu_{b, N A}(\mathrm{NA}=0.08)$. This leads to a larger variation in experimental results than can be explained by the variation in size distributions using Mie-theory.

This study gives a thorough overview of the optical properties of mature milk, which is produced by the mammary gland after approximately 2 weeks postpartum [31]. Up to 3-4 days postpartum, the mammary gland produces colostrum, followed by a period with transitional milk until mature milk is produced. The scattering properties of colostrum and transitional milk are expected to be different from mature milk, as fat globules in colostrum are smaller [36] (diameter of around $2 \mu \mathrm{m}$ ) and fat concentrations are lower [45]. Furthermore, the bright yellow appearance of colostrum implies that absorption also plays a more prominent role for wavelengths lower than 500 $\mathrm{nm}$, which is likely to be caused by higher beta-carotene concentrations $[31,46]$. Further research is required to map the optical properties of colostrum and transitional milk.

This study explored the interaction of light with human milk. We hope that our findings will form a starting point for further biophotonic studies on human milk and lactation, with potential applications ranging from human milk analysis, to studies on lactation physiology, and objective methods to support mothers who experience breastfeeding problems. As our findings indicate a significant correlation between fat concentration of human milk and its optical properties 
$\left(\mu_{a}, \mu_{s}, \mu_{s}^{\prime}\right.$ and $\left.\mu_{b, N A}\right)$ within the visible wavelength region, this can potentially lead to a cost-effective alternative for (near)-infrared analysis of human milk fat concentration.

\subsection{Conclusion}

In this study, we used a novel combination of spatially resolved diffuse reflectance spectroscopy and spectroscopic optical coherence tomography to quantify the optical properties of human foremilk, bulk milk and hindmilk between $450-650 \mathrm{~nm}$. The observed range of variation between subjects in the optical properties was $\mu_{a, 460 \mathrm{~nm}}=1.1 \times 10^{-2}-8.3 \times 10^{-2} \mathrm{~mm}^{-1}, \mu_{s, 550 \mathrm{~nm}}=2.6-$ $21.0 \mathrm{~mm}^{-1}, \mu_{s, 550 \mathrm{~nm}}^{\prime}=0.24-2.48 \mathrm{~mm}^{-1}, g=0.82-0.96$, and $\mu_{b, N A, 550 \mathrm{~nm}}=1.1 \times 10$ ${ }^{4}-1.6 \times 10^{-3} \mathrm{~mm}^{-1}$. Significant correlations with fat concentration were found for $\mu_{a}, \mu_{s}, \mu_{s}{ }^{\prime}$ and $\mu_{b, N A}\left(R_{s}=0.38, R_{s}=0.77, R_{s}=0.80\right.$, and $R_{s}=0.44$, respectively). Mie calculations on the measured fat globule size distribution of human milk were in good agreement with the experimentally derived optical properties. In conclusion, the variation in the optical properties of human milk between and within mothers can be deduced largely to biological variations in fat concentration and fat globule size distribution.

\section{Bibliography}

1. C. G. Victora, R. Bahl, A. J. D. Barros, G. V. A. Franca, S. Horton, J. Krasevec, S. Murch, M. J. Sankar, N. Walker, and N. C. Rollins, "Breastfeeding in the 21st century: epidemiology, mechanisms, and lifelong effect," Lancet 387(10017), 475-490 (2016).

2. N. C. Rollins, N. Bhandari, N. Hajeebhoy, S. Horton, C. K. Lutter, J. C. Martines, E. G. Piwoz, L. M. Richter, and C. G. Victora, "Why invest, and what it will take to improve breastfeeding practices?," Lancet 387(10017), 491504 (2016).

3. K. Hinde and J. B. German, "Food in an evolutionary context: insights from mother's milk," J Sci Food Agr 92(11), 2219-2223 (2012).

4. Unicef and World Health Organization "Tracking progress for breastfeeding policies and programmes: Global breastfeeding scorecard 2017.", Retrieved 12-01-2019, at https://www.who.int/nutrition/publications/infantfeeding/global-bfscorecard-2017/en/ 
5. R. W. Li, S. B. Fein, J. Chen, and L. M. Grummer-Strawn, "Why mothers stop breastfeeding: Mothers' self-reported reasons for stopping during the first year," Pediatrics 122, S69-S76 (2008).

6. M. C. Neville, S. M. Anderson, J. L. McManaman, T. M. Badger, M. Bunik, N. Contractor, T. Crume, D. Dabelea, S. M. Donovan, N. Forman, D. N. Frank, J. E. Friedman, J. B. German, A. Goldman, D. Hadsell, M. Hambidge, K. Hinde, N. D. Horseman, R. C. Hovey, E. Janoff, N. F. Krebs, C. B. Lebrilla, D. G. Lemay, P. S. MacLean, P. Meier, A. L. Morrow, J. Neu, L. A. Nommsen-Rivers, D. J. Raiten, M. Rijnkels, V. Seewaldt, B. D. Shur, J. VanHouten, and P. Williamson, "Lactation and Neonatal Nutrition: Defining and Refining the Critical Questions," J Mammary Gland Biol 17(2), 167-188 (2012).

7. K. Tanimoto, T. Kusaka, T. Nishida, K. Ogawa, I. Kato, S. Ijichi, J. Mikami, I. Sobue, K. Isobe, and S. Itoh, "Hemodynamic Changes in the Breast and Frontal Cortex of Mothers During Breastfeeding," Pediatr Res 70(4), 400405 (2011).

8. M. Eriksson, T. Lundeberg, and K. UvnasMoberg, "Studies on cutaneous blood flow in the mammary gland of lactating rats," Acta Physiol Scand 158(1), 1-6 (1996).

9. M. van der Hoek, L. den Haan, A. Kaspers, W. Steenbergen, and N. Bosschaart, "Cutaneous perfusion of the human lactating breast: a pilot study with laser Doppler perfusion monitoring," Physiol Meas 40(5), 05NT01 (2019).

10. N. Bosschaart, A. Leproux, O. Abdalsalam, W. Chen, C.E. McLaren, B.J. Tromberg, and T.D. O'Sullivan, "Diffuse optical spectroscopic imaging for the investigation of human lactation physiology: a case study on mammary involution," J Biomed Opt 24(5), 056006 (2019).

11. N. Argov, S. Wachsmann-Hogiu, S. L. Freeman, T. Huser, C. B. Lebrilla, and J. B. German, "Size-dependent lipid content in human milk fat globules," J Agr Food Chem 56(16), 7446-7450 (2008).

12. E. D. M. Motta, R. A. Zangaro, and L. Silveira, "Quantitative determination of the human breast milk macronutrients by near-infrared Raman spectroscopy," Proc Spie 8229, 82291F (2012). 
13. Y. P. Yao, G. Z. Zhao, Y. Y. Yan, H. Y. Mu, Q. Z. Jin, X. Q. Zou, and X. G. Wang, "Milk fat globules by confocal Raman microscopy: Differences in human, bovine and caprine milk," Food Res Int 80, 61-69 (2016).

14. R. Ullah, S. Khan, S. Javaid, H. Ali, M. Bilal, and M. Saleem, "Raman spectroscopy combined with a support vector machine for differentiating between feeding male and female infants mother's milk," Biomed Opt Express 9(2), 844-851 (2018).

15. G. Fusch, N. Rochow, A. Choi, S. Fusch, S. Poeschl, A. O. Ubah, S. Y. Lee, P. Raja, and C. Fusch, "Rapid measurement of macronutrients in breast milk: How reliable are infrared milk analyzers?," Clin Nutr 34(3), 465-476 (2015).

16. S. Stocker, F. Foschum, P. Krauter, F. Bergmann, A. Hohmann, C. S. Happ, and A. Kienle, "Broadband Optical Properties of Milk," Appl Spectrosc 71(5), 951-962 (2017).

17. E. Bijl, R. de Vries, H. van Valenberg, T. Huppertz, and T. Van Hooijdonk, "Factors influencing casein micelle size in milk of individual cows: Genetic variants and glycosylation of kappa-casein," Int Dairy J 34(1), 135-141 (2014).

18. L. R. Mitoulas, J. C. Kent, D. B. Cox, R. A. Owens, J. L. Sherriff, and P. E. Hartmann, "Variation in fat, lactose and protein in human milk over $24 \mathrm{~h}$ and throughout the first year of lactation," Brit J Nutr 88(1), 29-37 (2002).

19. B. Aernouts, R. Van Beers, R. Watte, T. Huybrechts, J. Jordens, D. Vermeulen, T. Van Gerven, J. Lammertyn, and W. Saeys, "Effect of ultrasonic homogenization on the Vis/NIR bulk optical properties of milk," Colloid Surface B 126, 510-519 (2015).

20. N. Bosschaart, D. J. Faber, T. G. van Leeuwen, and M. C. G. Aalders, "Measurements of wavelength dependent scattering and backscattering coefficients by low-coherence spectroscopy," J Biomed Opt 16(3), 030503 (2011).

21. M. Almasian, N. Bosschaart, T. G. van Leeuwen, and D. J. Faber, "Validation of quantitative attenuation and backscattering coefficient measurements by optical coherence tomography in the concentration-dependent and multiple scattering regime," J Biomed Opt 20(12), 121314 (2015). 
22. Choi, G. Fusch, N. Rochow, N. Sheikh, and C. Fusch, "Establishment of micromethods for macronutrient contents analysis in breast milk," Matern Child Nutr 11(4), 761-772 (2015).

23. T. J. Farrell, M. S. Patterson, and B. Wilson, "A Diffusion-Theory Model of Spatially Resolved, Steady-State Diffuse Reflectance for the Noninvasive Determination of Tissue Optical-Properties Invivo," Med Phys 19(4), 879888 (1992).

24. N. Bosschaart, R. Mentink, J. H. Kok, T. G. van Leeuwen, and M. C. G. Aalders, "Optical properties of neonatal skin measured in vivo as a function of age and skin pigmentation," J Biomed Opt 16(9), 097003 (2011).

25. N. Bosschaart, M. C. G. Aalders, T. G. van Leeuwen, and D. J. Faber, "Spectral domain detection in low-coherence spectroscopy," Biomed Opt Express 3(9), 2263-2272 (2012).

26. C. Veenstra, W. Petersen, I. M. Vellekoop, W. Steenbergen, and N. Bosschaart, "Spatially confined quantification of bilirubin concentrations by spectroscopic visible-light optical coherence tomography," Biomed Opt Express 9(8), 3581-3589 (2018).

27. C. F. Bohren, and D. R. Huffman, "Absorption and scattering of light by small particles" (Wiley-Interscience, 1998).

28. J. P. Schäfer, "Implementierung und Anwendung analytischer und numerischer Verfahren zur Lösung der Maxwellgleichungen für die Untersuchung der Lichtausbreitung in biologischem Gewebe," (Universität Ulm, 2011).

29. J. Jaaskelainen, K. E. Peiponen, and J. A. Raty, "On reflectometric measurement of a refractive index of milk," J Dairy Sci 84(1), 38-43 (2001).

30. R. Attaie and R. L. Richter, "Size distribution of fat globules in goat milk," J Dairy Sci 83(5), 940-944 (2000).

31. K. Wambach, and J. Riordan, Breastfeeding and Human Lactation, Enhanced 5th edn. (Jones \& Bartlett Learning, 2016), Chap. 4.

32. F. C. Cheong, K. Xiao, and D. G. Grier, "Technical note: Characterizing individual milk fat globules with holographic video microscopy," J Dairy Sci 92(1), 95-99 (2009). 
33. M. C. Michalski, V. Briard, and F. Michel, "Optical parameters of milk fat globules for laser light scattering measurements," Lait 81(6), 787-796 (2001).

34. L. J. Street, Introduction to Biomedical Engineering Technology $3^{\text {rd }}$ edn. (Taylor \& Francis/CRC Press, 2017), Chap 1.

35. R. Pérez-Escamilla, R. J. Cohen, K. H. Brown, L. L. Rivera, J. Canahuati, and K. G. Dewey, "Maternal anthropometric status and lactation performance in a low-income Honduran population: evidence for the role of infants," Am J Clin Nutr 61(3), 528-534 (1995).

36. M. Ruegg and B. Blanc, "The Fat Globule Size Distribution in Human-Milk," Biochim Biophys Acta 666(1), 7-14 (1981).

37. M. C. Michalski, V. Briard, F. Michel, F. Tasson, and P. Poulain, "Size distribution of fat globules in human colostrum, breast milk, and infant formula," J Dairy Sci 88(6), 1927-1940 (2005).

38. S. Prahl, "Beta-carotene", retrieved 18-01-2019, https://omlc.org/spectra/PhotochemCAD/html/041.html.

39. S. Prahl, "Riboflavin", retrieved 18-01-2019, https://omlc.org/spectra/PhotochemCAD/html/004.html.

40. S. Prahl, "Optical Absorption of Fat", retrieved 18-01-2019, https://omlc.org/spectra/fat/.

41. B. Aernouts, R. Van Beers, R. Watté, T. Huybrechts, J. Lammertyn, and W. Saeys, "Visible and near-infrared bulk optical properties of raw milk," J Dairy Sci 98(10), 6727-6738 (2015).

42. B. Y. Fong, C. S. Norris, and A. K. H. MacGibbon, "Protein and lipid composition of bovine milk-fat-globule membrane," Int Dairy J 17(4), 275288 (2007).

43. C. G. de Kruif, T. Huppertz, V. S. Urban, and A. V. Petukhov, "Casein micelles and their internal structure," Adv Colloid Interfac 171, 36-52 (2012).

44. K. S. Rangappa, "Studies on the refractive index of milk. 1. Observations on genuine samples," Proceedings of the National Academy of Sciences of India, Section B. Biological Sciences 25, 86-94 (1947).

45. C. Molto-Puigmarti, A. I. Castellote, X. Carbonell-Estrany, and M. C. LopezSabater, "Differences in fat content and fatty acid proportions among colostrum, transitional, and mature milk from women delivering very 
preterm, preterm, and term infants," Clinical Nutrition 30(1), 116-123 (2011).

46. A. O. Xavier, E. Diaz-Salido, I. Arenilla-Velez, J. Aguayo-Maldonado, J. Garrido-Fernandez, J. Fontecha, A. Sanchez-Garcia, and A. Perez-Galvez, "Carotenoid Content in Human Colostrum is Associated to Preterm/FullTerm Birth Condition," Nutrients 10(11), 1654 (2018). 


\section{Chapter 5:}

\section{The dependency of the optical scattering properties of human milk on casein content and common sample preparation methods}

Abstract: Quantifying human milk composition is important for daily nutritional management in neonatal intensive cares worldwide. Photonic solutions based on visible light can potentially aid in this analysis, as energy content of human milk depends largely on fat content, and the optical scattering properties of human milk predominantly depend on the size and concentration of fat globules. However, it is expected that human milk scattering changes upon homogenization, routinely done before analysis, which may affect fat globule size. The first aim of this study was to investigate how the most common homogenization methods (gently inverting by hand, vortexing, sonication) affect the optical properties of human milk. The second aim was to estimate the scattering contribution of casein micelles, the second most dominant scatterers in human milk. We combined diffuse reflectance spectroscopy with spectroscopic optical coherence tomography to measure the scattering coefficient $\mu_{s}$, reduced scattering coefficient $\mu_{s}$ ' and anisotropy $g$ between 450$600 \mathrm{~nm}$. Sonication induced the strongest changes in $\mu_{s}, \mu_{s}{ }^{\prime}$ and $g$ compared to the gently inverted samples $(203 \%, 202 \%$ and $7 \%$, respectively at $550 \mathrm{~nm})$, but also vortexing changed $\mu_{s}{ }^{\prime}$ with $20 \%$. Although casein micelles only showed a modest contribution to $\mu_{s}$ and $g$ at $550 \mathrm{~nm}$ ( $7 \%$ and $1 \%$ respectively), their contribution to $\mu_{s}{ }^{\prime}$ was $29 \%$. In conclusion, the scattering properties of human milk strongly depend on the homogenization method that is employed, and gentle inversion should be the preferred method. The contribution of casein micelles was relatively small for $\mu_{s}$ and $g$, but considerably larger for $\mu_{s}{ }^{\prime}$.

This chapter is published as: Colin Veenstra, Dayna E. Every, Wilma Petersen, Johannes B. van Goudoever, Wiendelt Steenbergen, and Nienke Bosschaart, "The dependency of the optical scattering properties of human milk on casein content and common sample preparation methods," J. Biomed. Opt. 25(4), 045001 (2020) 


\subsection{Introduction}

Human milk is the optimal source of nutrition for infants in early life [1,2]. Breastfeeding supports infant survival, growth, health and cognitive development [3] and protects against many types of infections, diarrhea and dental malocclusions [1]. Also mothers benefit from breastfeeding with a reduced risk for breast cancer, ovarian cancer and improved birth spacing [1]. As a consequence, it is estimated that the deaths of 823,000 children and 20,000 mothers can be prevented every year if all mothers worldwide would breastfeed their infants [1].

Despite all these advantages, breastfeeding rates - especially in high-income countries - do not meet the advice of the World Health Organization for mothers to exclusively breastfeed their infants until the age of 6 months, followed by continued breastfeeding with complimentary foods until a minimum age of 2 years [4]. Major reasons for mothers to stop breastfeeding are pain due to breastfeeding problems and (the perception of) insufficient milk supply [5]. As we have detailed in our recent work [6], photonics offers many valuable opportunities for the development of more objective tools for lactation science and support. These tools can be employed to obtain unique insights into the physiology of the lactating breast [7-9], but also as an efficient method to quantify human milk composition [10-12]. The latter is particularly important for monitoring the nutritional intake of premature newborns, who commonly require human milk fortification with additional nutrients to ensure optimal development. This patient group will likely benefit from a more personalized fortification approach, rather than the currently applied one-size-fits-all fortification that neglects the differences in patient specific milk intake and composition [11,13]. To introduce and employ photonic methods for quantification of human milk composition, detailed knowledge on the interaction between human milk and light is required.

To evaluate milk composition in dairy industry, commercially available bovine milk analyzers estimate fat, protein and lactose concentrations through near (NIR) and mid infrared (MIR) optical absorption spectroscopy [14]. In the past decade, these analyzers have been adapted to quantify fat and protein concentrations in human milk with reasonable accuracy [11]. As NIR and MIR human milk analyzers rely on relatively costly optical components, optical methods employing the visible wavelength range may offer a cost-effective 
alternative. Reducing costs may make milk analysis more widely available to support more mothers who need feedback on the nutritional intake of their infant.

In the visible wavelength range, the optical attenuation by milk is dominated by the scattering of fat globules ( 0.2-11 $\mu \mathrm{m}$ in diameter) [6]. In our recent work, we mapped the full set of optical properties of human milk and demonstrated significant correlations between milk fat concentration and the absorption coefficient $\mu_{a}$, scattering coefficient $\mu_{s}$, reduced scattering coefficient $\mu_{s}{ }^{\prime}$ and backscattering coefficient $\mu_{b, N A}$ [6]. Samples were homogenized through gently inverting by hand, to ensure that the fat globule size distribution was not affected by the sample preparation method. Building upon our previous work, this work aims to investigate the effect of other common and time-efficient sample preparation methods on the scattering properties of human milk: vortexing and sonication. This is essential knowledge for any future work on the optical investigation of human milk. Sonication is a crucial preparation step for NIR and MIR human milk analyzers, as it reduces the average size of the milk fat globules, and thereby reduces the cross-talk from optical scattering to the measured absorption $[11,15,16]$. Human milk sonication methods described in literature are highly variable in terms of sonication power and duration $[11,15,16]$.

Next to fat globules, casein micelles (with radii around $100 \mathrm{~nm}$ ) are the second most dominant scattering particles in human milk. Therefore, the second aim of this study is to also quantify their contribution to the scattering properties of human milk. Numerical results have previously shown that - due to their small size - casein micelles have a relatively small effect on $\mu_{s}(\sim 6 \%)$, whereas they do notably affect $\mu_{s}^{\prime}$ ( $43 \%$, depending on fat concentration) [6]. Knowledge on the contribution of casein to the optical scattering properties of human milk is therefore relevant for both the quantification of fat content, and the casein content itself.

In this work, we quantified the scattering properties of human milk, by using our existing combination of spatially resolved diffuse reflectance spectroscopy (SR-DRS) and spectroscopic optical coherence tomography (sOCT) [6]. Human milk samples were homogenized with four different methods: 1) gently inverting the milk samples by hand, 2) vortexing, 3) sonication for one minute, 4) sonication for five minutes. The contribution of casein micelles was estimated 
by measuring the scattering properties before and after denaturation of the casein micelles. We explain our experimental results with Mie-theory, by modelling milk as a suspension of fat globules and casein micelles in whey.

\subsection{Materials and methods}

\subsubsection{Sample collection procedure}

For this study, the DHMB (Dutch Human Milk Bank, Amsterdam, The Netherlands) provided mature human milk samples from five healthy donors with a lactation period between 2 and 8 months postpartum. All donors signed informed consent. The donors collected the milk samples between 21 January 2018 and 15 March 2018 at home with a breast milk pump, using standardized procedures. Immediately after collecting the milk samples in disposable bisphenol A-free bottles (Sterifeed, Medicare Colgate Ltd, Devon, England), the milk was stored in the donor's home freezer at $-18{ }^{\circ} \mathrm{C}$ to $-20{ }^{\circ} \mathrm{C}$. A carrier transported the milk to the $\mathrm{DHMB}$ for storage in a $-20^{\circ} \mathrm{C}$ freezer, before the milk was transported to the University of Twente. There, the milk was stored at -20 ${ }^{\circ} \mathrm{C}$ for a maximum period of 11 months post expression. Prior to the experiments, the samples were thawed in a $20{ }^{\circ} \mathrm{C}$ water bath. Fat concentrations ranged from $21.5-41.3 \mathrm{~g}$ per $\mathrm{kg}$ milk, as we determined by a modified Mojonnier method [6,17].

\subsubsection{Preparation methods}

To study the dependency of the scattering properties of milk on the sample preparation method, we created five milk samples per donor by pipetting $5 \mathrm{~mL}$ of milk into five separate $10 \mathrm{~mL}$ falcon tubes. Hereafter, four samples were homogenized by four different methods: 1) Gently inverting the sample by hand. 2) Vortexing the sample for 10 seconds (Vortex-Genie 2, Scientific Industries, USA, Power 10). 3) Sonication of the sample for 1 minute (Sonifier 250, Branson, USA, Power 3, Duty cycle $30 \%$ ). 4) Sonication of the sample for 5 minutes with the same settings. During sonication, samples were cooled by placing the falcon tube into an ice bath. Performing these preparation methods resulted into four samples per donor.

The fifth milk sample was used to investigate the contribution of casein micelles to the scattering properties of milk. Hereto, we adapted the method from Stocker, et al. [15] to denature the casein micelles by adding an excess of $75 \mu \mathrm{L}$ 
of $0.5 \mathrm{M}$ ethylenediaminetetraacetic acid (EDTA) to the $5 \mathrm{~mL}$ milk sample. This denatures the casein micelles by destroying the calcium phosphate nanoclusters inside the casein micelles [18]. After denaturation, casein micelle scattering becomes negligible [15]. The samples containing EDTA were homogenized by gently inverting the sample by hand. Following all sample preparation methods described above, we prepared 5 samples per donor, yielding a total of 25 samples.

High concentrations of scattering particles may hinder the quantification of the scattering properties due to strong loss of signal in the SR-DRS setup and the contribution of multiply scattered light to the SOCT signal. Therefore, milk samples with an initial measured $\mu_{s}>6 \mathrm{~mm}^{-1}$ or $\mu_{s}{ }^{\prime}>1 \mathrm{~mm}^{-1}$ at $550 \mathrm{~nm}$ were diluted with phosphate buffered saline until the results were below the mentioned values. Assuming concentration-independent single scattering events, the $\mu_{s}$ and $\mu_{s}$ ' of the original milk sample were retrieved by rescaling these scattering properties with the dilution factor. All preparation methods were applied before dilution of the samples. All optical measurements in this work were performed in triplo. Hereto, each sample was measured three times, while moving the sample in and out of the experimental setup in between consecutive measurements. Unless stated otherwise, all reported values are averages of the triplo measurement.

\subsubsection{Bright-field microscopy}

To study the effect of preparation method on the fat globules in the milk, all samples were imaged with bright-field microscopy (EVOS FL Cell Imaging System, Thermo Fisher, USA) at three different locations inside the sample. Hereafter, the fat globule size distributions were obtained by processing the images with Matlab (R2017b, MathWorks, USA) and its built-in 'imfindcircles' function.

\subsubsection{Experimental estimation of the scattering properties}

To experimentally estimate the scattering properties of all samples, we used the same methods as in our previous work [6]. Hereto, we combined spatially resolved diffuse reflectance spectroscopy (SR-DRS) and spectroscopic optical coherence tomography (sOCT) to obtain the scattering properties $\left(\mu_{s}, \mu_{s}{ }^{\prime}\right.$ and $g$ ) of the milk samples. A schematic overview of the set-ups and a flow chart of the 
methods is given in figure 5.1 and a brief description of the methods is given below.

\subsubsection{Spatially resolved diffuse reflectance spectroscopy}

The reduced scattering coefficient $\left(\mu_{s}{ }^{\prime}\right)$ of the milk samples was estimated by SR-DRS. We used a fiber-based home-built SR-DRS system that measured the diffuse reflectance as a function of inter-fiber distance $r$ by translation of the detection fiber. The inter-fiber distance was varied from $r_{0}=2.2 \mathrm{~mm}$ to $r_{\text {end }}=4.7$ $\mathrm{mm}$ in steps of $50 \mu \mathrm{m}$. The integration time was varied between samples and was set such that at $r_{0}, 80 \%$ of the dynamic range of the spectrometer was filled. The dark-noise was obtained by a measurement without illumination.

The reduced scattering coefficient was obtained by adapting the model from Farrell, et al. [19]. This model describes the diffuse reflectance as a function of the inter-fiber distance and the optical properties $\mu_{s}{ }^{\prime}$ and $\mu_{a}$ :

$$
R_{\text {theory }}(r)=\frac{1}{4 \pi}\left(\frac{z_{0}}{r_{1}^{2}}\left(\mu_{e f f}+\frac{1}{r_{1}}\right) e^{-\mu_{e f f} r_{1}}+\frac{z_{0}+2 z_{b}}{r_{2}^{2}}\left(\mu_{e f f}+\frac{1}{r_{2}}\right) e^{-\mu_{e f f} r_{2}}\right)
$$

With effective attenuation coefficient $\mu_{\text {eff }}=\left(3 \mu_{a}\left(\mu_{a}+\mu_{s}^{\prime}\right)\right)^{1 / 2}$, inverse total interaction coefficient $z_{0}=\left(\mu_{a}+\mu_{s}^{\prime}\right)^{-1}, r_{1}=\left(z_{0}^{2}+r^{2}\right)^{1 / 2}, r_{2}=\left(\left(z_{0}+2 z_{b}\right)^{2}\right)^{1 / 2}$, and $z_{b}=$ (2/3) $\left.\left(\mu_{a}+\mu_{s}\right)^{\prime}\right)^{-1}$, under the conditions that $r>1 /\left(\mu_{s}^{\prime}+\mu_{a}\right)$ and $\mu_{s}{ }^{\prime}>>\mu_{a}$. For wavelengths smaller than $530 \mathrm{~nm}$, absorption starts to play a more prominent role in the measured diffuse reflectance [6]. Therefore, we follow our approach in Bosschaart, et al. [20] by using a more robust two-step fitting approach over the wavelength range with $(\lambda<530 \mathrm{~nm})$ and $(\lambda>530 \mathrm{~nm})$ without absorption. Hereto, we first obtain $\mu_{s}{ }^{\prime}$ for $\lambda>530 \mathrm{~nm}$ by fitting Eq. 5.2 to the dark noise corrected $R_{\text {meas }}(r)$ under the assumption of negligible absorption in this wavelength range:

$$
R_{\text {meas }}(r)=\beta R_{\text {theory }}(r)
$$

with $\mu_{a}=0$ and fit parameters: scaling factor $\beta$ and $\mu_{s}^{\prime}$. If the model validity condition $\left(r>1 / \mu_{s}{ }^{\prime}\right.$ with $\left.\mu_{a}=0\right)$ was not satisfied, $r_{0}$ was increased until the 
condition was satisfied. Hereafter, $\mu_{s}^{\prime}$ was estimated for $\lambda<530 \mathrm{~nm}$ by extrapolating a fitted power law to the results obtained from Eq. 5.2:

$$
\mu_{s}{ }^{\prime}=a \lambda^{-b}
$$

with fit parameters: scaling factor $a$ and scatter power $b$. Finally, we estimated $\mu_{a}$ by fitting Eq. 5.2 to $R_{\text {meas }}(r)$ over the wavelength range of $450-600 \mathrm{~nm}$, with $\mu_{s}{ }^{\prime}$ fixed at the previously obtained values and fit parameters $\mu_{a}$ and $\beta$.

\subsubsection{Spectroscopic optical coherence tomography}

SOCT is an optical technique that allows for quantitative and localized measurements of total attenuation, scattering and absorption coefficients, as we have shown in our previous work for a wide range of samples ( $\mu_{t}$ ranging from 0.15 to $34 \mathrm{~mm}^{-1}$ ) [21-24]. Here, we used SOCT to estimate the scattering coefficient $\left(\mu_{s}\right)$ of the milk samples.

We used a broadband Michelson-interferometer based SOCT system (figure 5.1b), which measured the interference between the backscattered light from the sample and reference arm. A detailed description of our SOCT system is given in Veenstra, et al. [24]. In short, we measured the backscattered spectra from the sample as a function of depth $d$. Our combined approach of focus tracking and zero-delay acquisition ensured that the measured attenuation of the OCT-signal with depth is affected only by the optical properties of the sample. The acquired spectra were short-time Fourier transformed with a spectral resolution of $5 \mathrm{~nm}$ resulting in a spatially and spectrally confined dataset of the backscattered intensity from the sample $S(\lambda, d)$ with a spatial resolution ranging from $15 \mu \mathrm{m}$ (at $\lambda=450 \mathrm{~nm}$ ) to $27 \mu \mathrm{m}$ (at $\lambda=600 \mathrm{~nm}$ ). The lateral resolution of our system was $2.5 \mu \mathrm{m}$ in air.

Under the assumption of single scattering, the attenuation spectrum $\left(\mu_{t}=\mu_{a}+\right.$ $\mu_{s}$ ) was obtained by fitting Lambert-Beer's law to $S(\lambda, d)$ for every wavelength in the range of $450-600 \mathrm{~nm}$ :

$$
\ln \left(\left(S(\lambda, d)-S_{b g}(\lambda)\right)^{2}\right)=\ln (\alpha(\lambda))-2 \mu_{t}(\lambda) d
$$

with scaling factor $\alpha$ and $\mu_{t}$ as fit parameters. The background term $S_{b g}(\lambda)$ was acquired by performing a measurement at a depth of $1 \mathrm{~mm}$ inside the sample, at which the OCT-signal had been completely attenuated. Hereafter, we 
quantified $\mu_{s}$ by correcting $\mu_{t}$ with the $\mu_{a}$ results from the SR-DRS measurement: $\mu_{s}=\mu_{t}-\mu_{a}$. Finally, the anisotropy $g$ was estimated by combining the results of $\mu_{s}$ and $\mu_{s}^{\prime}$ :

$$
g=1-\frac{\mu_{s}{ }^{\prime}}{\mu_{s}}
$$

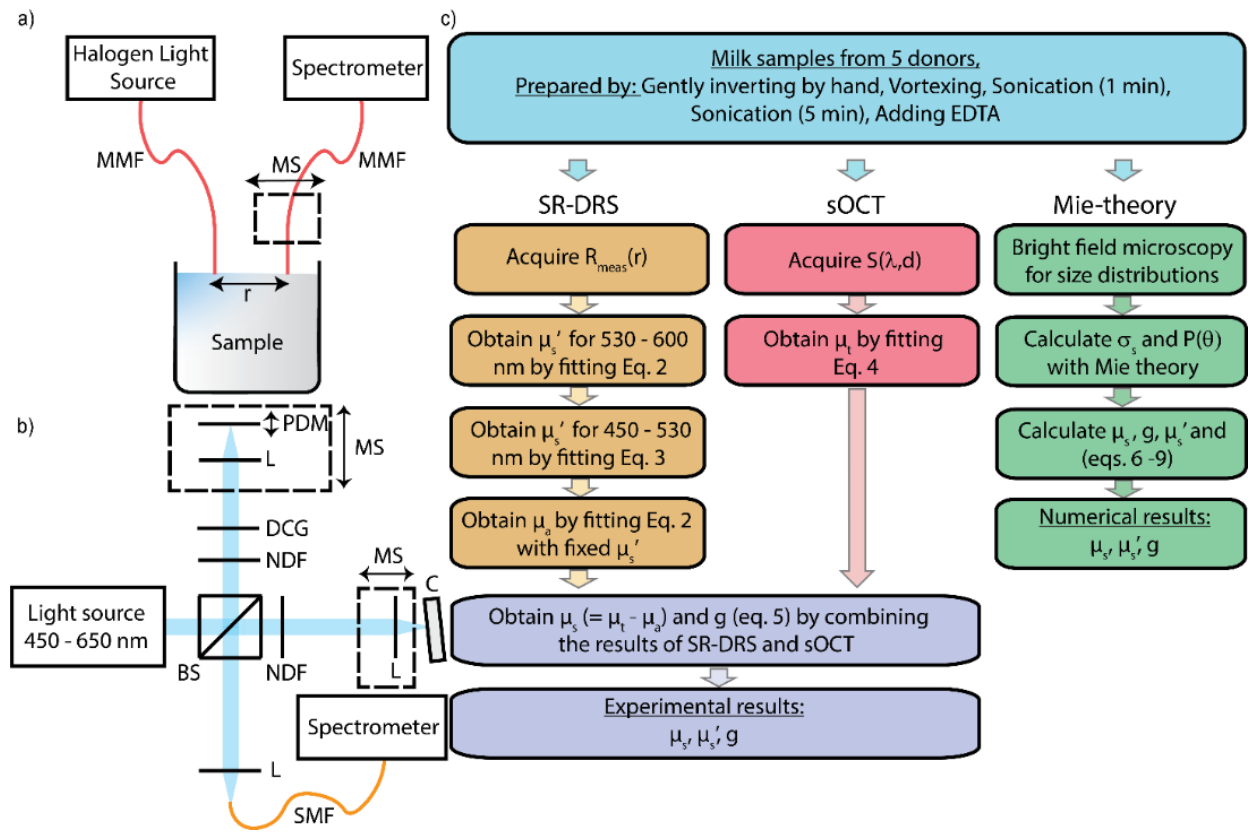

Figure 5.1: Schematic overview of the techniques used to estimate the scattering properties. a) Illustration of the SR-DRS setup. Light from the illumination fiber diffuses through the sample, which was detected as a function of inter-fiber distance $r$ by translation of the detection fiber. b) Schematic overview of the SOCT setup. c) Flowchart of the experimental and numerical methods in this study and the scattering properties they provide. MMF: multi mode fiber, $r$ : source-detector distance, MS: motorized stage, NDF: neutral density filters, L: lens, BS: beam splitter, DCG: dispersion compensation glass, PDM: piezo driven mirror, C: cuvette with sample, SMF: single mode fiber. 


\subsubsection{Methods accuracy}

As shown in our previous work, the accuracy of sOCT for estimating $\mu_{t}$ is approximately $10 \%$ [22]. The accuracies of SR-DRS for estimating $\mu_{a}$ and $\mu_{s}{ }^{\prime}$ are approximately $15 \%$ and $10 \%$, respectively [20]. Since we obtained $\mu_{s}$ and $g$ by combining the results from SOCT and SR-DRS, the inaccuracies of both methods propagate into the results for scattering properties. For human milk, $\mu_{a}$ is approximately 2 orders of magnitude smaller than $\mu_{s}$ [6]. Therefore, errors in the estimation of $\mu_{a}$ will be negligible for estimating $\mu_{s}$ through $\mu_{s}=\mu_{t}-\mu_{a}$. Since $g$ is obtained from the ratio of $\mu_{s}$ and $\mu_{s}{ }^{\prime}$, errors from both the SR-DRS and SOCT measurements propagate into $g$, resulting in an accuracy [6] of $14 \%$ for $1-g$.

\subsubsection{Numerical estimation of the scattering properties}

To validate and explain our experimental results, Mie-theory was used to theoretically investigate the influence of sample preparation method on the scattering properties of the milk samples. With the fat globule size distributions from the bright field microscopic measurements as an input, we modeled milk as a suspension of fat globules with variable radius $r_{f a t}$ (refractive index $n_{f a t}=$ $1.46[25,26])$ and monodisperse casein micelles in whey $\left(n_{\text {whey }}=1.345\right.$ [27]). Since literature values for casein in human milk were unavailable, values of bovine milk were used [28,29]: $n_{\text {casein }}=1.5$ and $r_{\text {casein }}=100 \mathrm{~nm}$. We used the MatScat software $[30,31]$ to individually calculate the scattering efficiencies $Q_{s}$ and phase functions $P(\vartheta)$ of casein micelles and fat globules - with $r_{f a t}$ ranging from 0 to $5 \mu \mathrm{m}-$ in whey at a wavelength of $550 \mathrm{~nm}$. Subsequently, we calculated $\mu_{s}$ by multiplying $Q_{s}$ with the cross sectional area $A=\pi r^{2}$ and the number of particles per volume $N=C /\left[(4 / 3) \pi r^{3}\right]$ with $C$ the volume fraction of either fat or casein in the milk:

$$
\mu_{s, \text { Mie }}\left(r_{\text {fat }}\right)=Q_{s, f a t}\left(r_{\text {fat }}\right) A_{f a t}\left(r_{\text {fat }}\right) N_{f a t}\left(r_{\text {fat }}\right)+Q_{s, c a s} A_{c a s} N_{c a s}
$$

All fat globule related parameters are a function of the measured fat globule size distribution $r_{\text {fat }}$, as Mie calculations were performed for the complete range of encountered fat globule radii. Since we investigated the influence of fat globule radius - and not fat concentration - on the scattering properties, we fixed the volume fractions at 0.325 ( $29 \mathrm{~g}$ fat per $\mathrm{kg}$ milk) for fat - which is the 
median fat volume fraction of the investigated milk samples in this study. The volume fraction of casein was fixed to a typical value [32] of $1.68 \times 10^{-3}$. To calculate the anisotropy $g\left(r_{f a t}\right)$, we first created a combined phase function by summing the unnormalized phase functions of fat globules and casein micelles:

$$
P_{c o m}\left(\theta, r_{f a t}\right)=\frac{N_{\text {fat }} P_{\text {fat }}\left(\theta, r_{\text {fat }}\right)+N_{c a s} P_{c a s}(\theta)}{N_{\text {total }}}
$$

after which $g\left(r_{f a t}\right)$ was calculated by:

$$
g_{\text {Mie }}\left(r_{\text {fat }}\right)=\frac{2 \pi \int_{0}^{\pi} P_{c o m}\left(\theta, r_{\text {fat }}\right) \cos \theta \sin \theta d \theta}{2 \pi \int_{0}^{\pi} P_{\text {com }}\left(\theta, r_{\text {fat }}\right) \sin \theta d \theta}
$$

finally, $\mu_{s}^{\prime}\left(r_{f a t}\right)$ can be obtained by combining the results for $\mu_{s}$ and $g$ :

$$
\mu_{s \text { Mie }}^{\prime}\left(r_{\text {fat }}\right)=\mu_{s, \text { Mie }}\left(r_{\text {fat }}\right)\left[1-g_{\text {Mie }}\left(r_{\text {fat }}\right)\right]
$$

to compare the experimental results of the milk samples containing EDTA (i.e. with the casein micelles denaturated), all Mie calculations were also performed for $N_{\text {cas }}=0$.

\subsection{Results}

\subsubsection{Bright field microscopy and fat globule size distributions}

Figure 5.2 shows typical bright field microscopy images for the four different sample homogenization methods and the addition of EDTA. The images demonstrate that homogenization by sonication reduces the size of the fat globules inside the milk samples. The fat globule size distributions (figure 5.3) show similar results for the gently inverted, vortexed and EDTA samples with a maximum around $4.5 \mu \mathrm{m}$. The size distributions of the samples that were sonicated for 1 minute have a maximum at a diameter of $1.75 \mu \mathrm{m}$. Due to the limited resolution $(\approx 0.75 \mu \mathrm{m})$ of the bright-field microscope, no fat globule size distribution could be obtained for the samples that were sonicated for 5 minutes. The arrows in figure 5.3 indicate that these samples therefore only contain particles $<0.75 \mu \mathrm{m}$. 

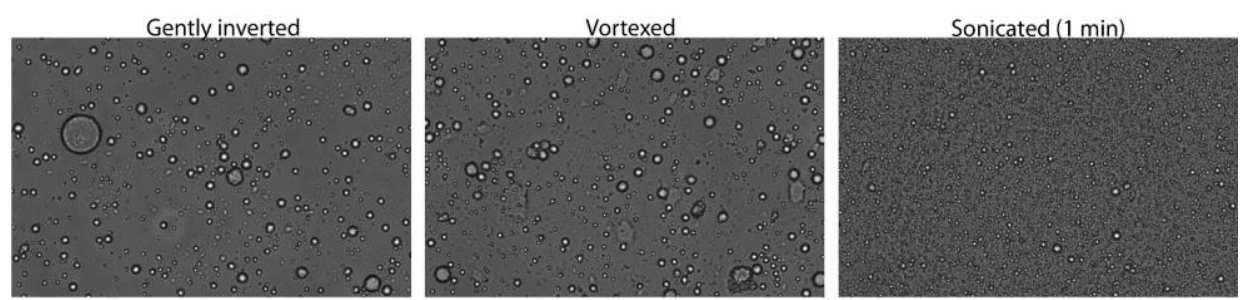

Sonicated $(5 \mathrm{~min})$

Containing EDTA (casein micelles denaturated)
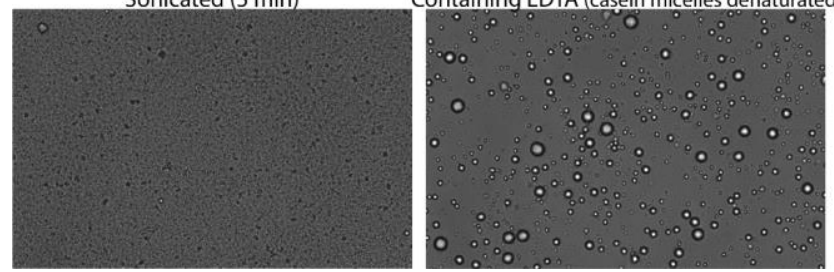

$$
100 \mu \mathrm{m}
$$

Figure 5.2: Typical bright field microscopy images of samples prepared with the different methods used in this work.

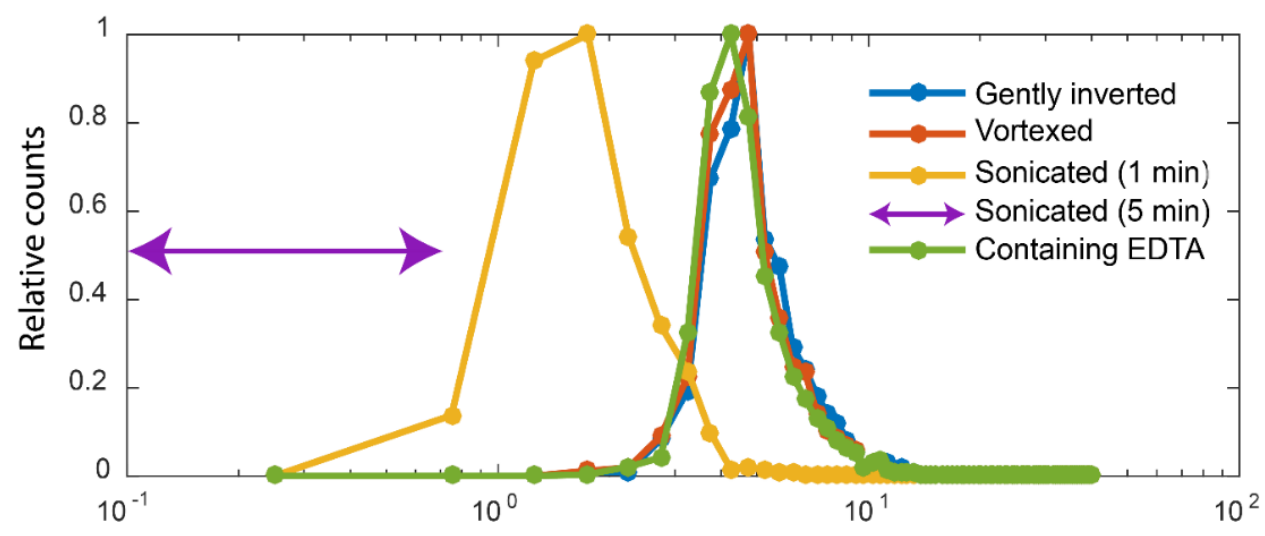

Fat globules diameter $(\mu \mathrm{m})$

Figure 5.3: Fat globule size distributions for the different sample preparation methods used in this work. The average distributions of all participants are shown and lines serve as a guide to the eye. Due to the resolution limit of the bright field microscope, the exact distribution of the samples that were sonicated for 5 minutes was not obtained. The arrows indicate that these samples therefore only contain particles $<0.75 \mu \mathrm{m}$. 


\subsubsection{Optical properties: Experimental results}

The median values of the measured optical property spectra for all milk samples and processing methods are shown in figure 5.4 for the wavelength range of $450-600 \mathrm{~nm}$. The median values and full range of the optical properties at 550 $\mathrm{nm}$ are listed in Table 5.1. All values mentioned in this section refer to the median values at $550 \mathrm{~nm}$.

The results for $\mu_{s}$ were similar for the gently inverted, vortexed and EDTA containing samples. Relative to the gently inverted samples, 1 minute of sonication resulted in a $203 \%$ increase in median $\mu_{s}$, whereas 5 minutes of sonication showed a $111 \%$ increase for $\mu_{s}$.

The vortexed and EDTA containing samples showed a $20 \%$ and $29 \%$ lower median $\mu_{s}^{\prime}$ than the gently inverted samples, respectively. Compared to gently inverting, 1 minute of sonication resulted in a $108 \%$ increase for $\mu_{s}^{\prime}$, while 5 minutes of sonication resulted in an even stronger increase of $202 \%$.

The vortexed samples showed higher anisotropy than the gently inverted samples. The samples that received 1 minute of sonication showed the highest $g$ with a value of 0.93 , whereas the samples receiving 5 minutes of sonication with a value of 0.84 showed the lowest $g$. The samples that were vortexed $(g=$ $0.92)$ or containing EDTA $(g=0.91)$ showed a slight increase in anisotropy compared to the gently inverted samples $(g=0.90)$.
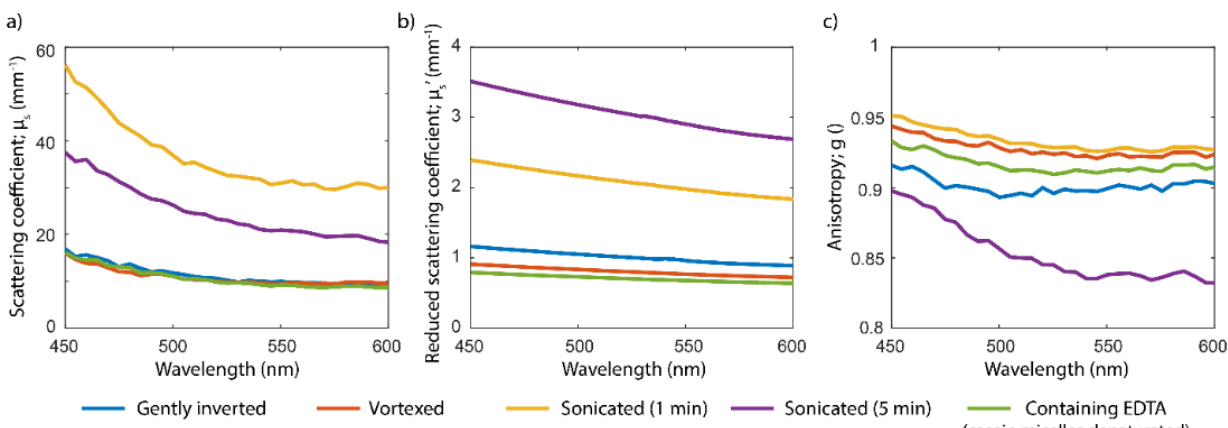

Figure 5.4: Experimentally derived scattering property spectra (medians of all donors) for all preparation methods. a) Scattering coefficient $\mu_{s .}$ b) Reduced scattering coefficient $\mu_{s}^{\prime}$. c) Anisotropy $g$. 
Table 5.1: Experimentally derived scattering properties at $\lambda=550 \mathrm{~nm}$. Results are shown as "median (full range)" for all samples.

\begin{tabular}{llll}
\hline $\begin{array}{l}\text { Preparation } \\
\text { method }\end{array}$ & $\boldsymbol{\mu}_{\mathrm{s}}\left(\mathrm{mm}^{-1}\right)$ & $\boldsymbol{\mu}_{\mathrm{s}}^{\prime}\left(\mathrm{mm}^{-1}\right)$ & $\mathbf{g}()$ \\
\hline Gently inverted & $9.9(5.1-11.2)$ & $0.96(0.64-2.1)$ & $0.90(0.81-0.91)$ \\
Vortexed & $9.4(6.0-10.8)$ & $0.77(0.40-1.2)$ & $0.92(0.89-0.93)$ \\
Sonicated (1 $\mathbf{~ m i n})$ & $30.0(20.2-40.8)$ & $2.0(1.5-2.7)$ & $0.93(0.92-0.94)$ \\
Sonicated (5 $\mathbf{m i n})$ & $20.9(16.3-23.7)$ & $2.9(1.9-4.5)$ & $0.84(0.81-0.90)$ \\
Containing EDTA & $9.2(5.4-10.7)$ & $0.68(0.47-0.99)$ & $0.91(0.88-0.93)$
\end{tabular}

\subsubsection{Optical properties: Numerical results}

Figure 5 shows the numerical results obtained by Mie-theory (solid lines) together with the median experimental results for comparison at $\lambda=550 \mathrm{~nm}$ (data points). All scattering properties are presented as a function of fat globule diameter. Similar to our experimental results, Mie theory predicts a modest contribution of casein micelles to the scattering coefficient of $0.51 \mathrm{~mm}^{-1}$ (figure 5.5a). Furthermore, Mie calculations show that for fat globules up to $2.2 \mu \mathrm{m}, \mu_{\mathrm{s}}$ increases with fat globule size. Hereafter, $\mu_{s}$ decreases until a local minimum is reached at $6.1 \mu \mathrm{m}$. Whereas numerical results overestimate the experimentally obtained $\mu_{s}$ values, numerical and experimental results do show the same trend for $\mu_{s}$ as a function of fat globule diameter.

The Mie calculated $\mu_{s}^{\prime}$ increases with fat globule diameter up to a diameter of $210 \mathrm{~nm}$, after which the $\mu_{s}{ }^{\prime}$ starts to decrease with fat globule diameter (figure 5.5b). Mie calculations show a casein micelle contribution of $0.30 \mathrm{~mm}^{-1}$ to the total $\mu_{s}^{\prime}$. The numerical and experimental results for $\mu_{s}{ }^{\prime}$ are in good agreement.

The anisotropy of casein micelles was calculated to be 0.41 . The Miecalculations show a maximum of $g=0.98$ for milk consisting of fat globules with a diameter of $2.6 \mu \mathrm{m}$ and a local minimum at $g=0.93$ for fat globules $5.9 \mu \mathrm{m}$ in diameter (figure 5.5c). The relative contribution of casein micelles is more pronounced for larger fat globules. Although the numerical results for $g$ 

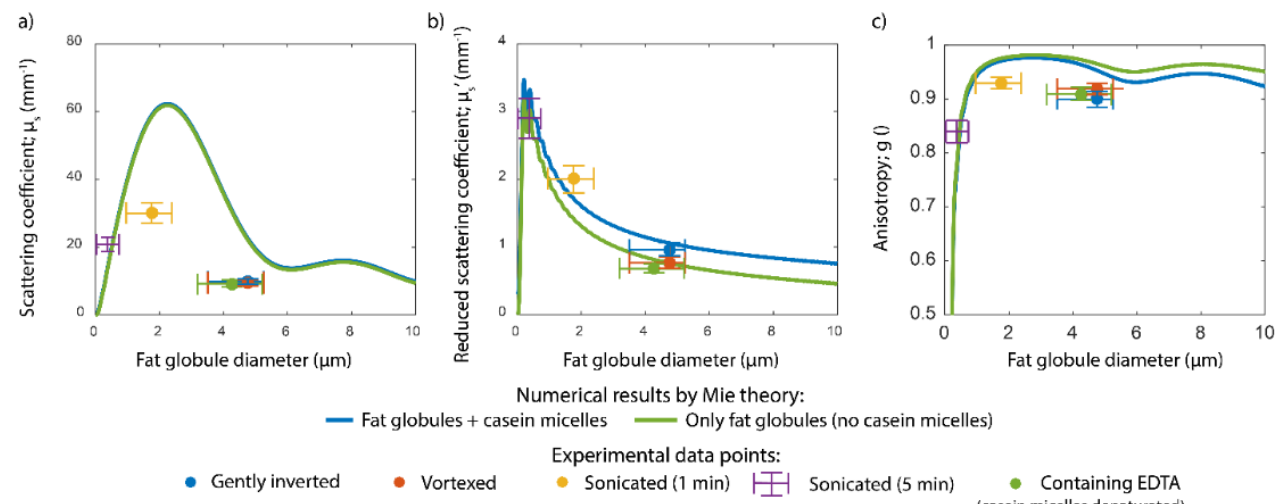

Numerical results by Mie theory:

Experimental data points:

- Sonicated (1 min) II Sonicated (5 min) Containing EDTA

Figure 5.5: Numerical results from Mie-theory for the scattering properties of milk containing $32.5 \mathrm{ml} / \mathrm{L}$ of monodisperse fat globules both with and without casein micelles as a function of fat globule diameter. The concentration (1.68 $\mathrm{ml} / \mathrm{L})$ and particle radius $(100 \mathrm{~nm}$ ) of the casein micelles were constant for all Mie-calculations. Experimental results are shown for comparison; the horizontal position of the dots and horizontal error bars indicate the mode diameter and the full width half maximum of the corresponding fat globule size distribution respectively, whereas the vertical position of the dots and vertical error bars indicate the median experimental values and accuracies (as described in section 5.2.4.3), respectively. Since a fat globule size distribution could not be obtained for the samples that were sonicated for 5 minutes, the horizontal error bars for this sample show the region of fat globule diameters lower than the microscope's resolution. a) Scattering coefficient $\mu_{s}$. b) Reduced scattering coefficient $\mu_{s}^{\prime}$. c) Anisotropy $g$.

overestimate the experimental results, they do show the same trend with fat globule diameter.

\subsection{Discussion}

In this study, we measured the influence of common sample preparation methods and casein content on the scattering properties of human milk. As a validation, our experimental results were compared to Mie theory.

In general, we can conclude that sonication alters the scattering properties of the milk more strongly than vortexing or gentle inversion. Due to a reduction in fat globule size, both $\mu_{s}$ and $\mu_{s}^{\prime}$ increase with respect to the gently inverted samples. Whereas the increase in $\mu_{s}$ is strongest for the samples that underwent 
1 minute of sonication, the increase in $\mu_{s}^{\prime}$ is strongest for the samples that underwent 5 minutes of sonication (Figure 5.4). This increase in $\mu_{s}$ and $\mu_{s}{ }^{\prime}$ after sonication was also consistently observed within samples from individual donors. Qualitatively, this agrees well with our Mie calculations that show a maximum for $\mu_{s}$ around the fat globule size distribution of the samples that were sonicated for 1 minute (Figure 5.5). Also the Mie calculated values for $g$ show a maximum around the fat globule size distribution of the samples that underwent 1 minute of sonication, which explains the experimental results for g. Our experimental results for sonicated samples agree well with the study on bovine milk from Stocker, et al. [15], who measured the same trends for $\mu_{s}$ and $\mu_{s}{ }^{\prime}$ with sonication time.

Compared to gently inverting, we observed a $20 \%$ reduction in $\mu_{s}^{\prime}$ for the vortexed samples, whereas $\mu_{s}$ only showed a decrease of $5 \%$. Since the fat globule size distributions of the gently inverted and vortexed samples are similar (Figure 5.3), the decrease in $\mu_{s}{ }^{\prime}$ cannot be ascribed to a difference in fat globule size. However, visual inspection of the vortexed milk samples revealed the presence of aggregates, which can be formed by the flocculation or coalescence of colliding fat globules during the vortexing process [33]. As SRDRS probes a many times larger volume compared to $\mathrm{SOCT}$, it is more likely that these aggregates will be present in the SR-DRS probing volume. As a consequence, they will have a larger effect on the measurement of $\mu_{s}{ }^{\prime}$ compared to that of $\mu_{s}$.

Although the trends between the experimental and numerical results for the different sample preparation methods are similar, our Mie calculations tend to overestimate the experimental results for $\mu_{s}$ and $g$ in absolute terms. Potential causes are: I) Mie theory assumes scattering by homogeneous spheres. This is a simplified model of fat globules as they consist of a lipid core surrounded by a phospholipid membrane [34]. II) We used the refractive index of fat globules in bovine milk as input parameter for the Mie calculations. The currently unreported refractive index of fat globules in human milk may be different. III) The refractive index of whey is assumed to be constant, while variations in the composition of the whey will affect the whey's refractive index. For skimmed bovine milk, the refractive index has been reported [35] to range between 1.345 and 1.348. IV) Inaccuracies in the chemically derived fat concentrations [6], which were used as an input parameter for Mie calculations. V) The use of 
bright-light microscopy can induce inaccuracies in the fat globule size distributions that were used to compare our experimental results with the Mie calculations (Figure 5.5). In bright field microscopy, a trade-off exists between the visibility of smaller and larger fat globules due to the limited depth of focus of the imaging plane. Out of focus fat globules will appear larger in the microscopic image than their actual size, which induces a right-shift of the resulting fat globule size distribution. As we used a bin size of $0.5 \mu \mathrm{m}$ to create the size distributions, this effect will be most prominent for the fat globule size distributions that contain the smallest particles.

Regarding the contribution of casein micelles to the scattering properties of human milk, the experimental and numerical results agree well on a quantitative level for $\mu_{s}^{\prime}$. At $\lambda=550 \mathrm{~nm}$, the absolute values for $\mu_{s}^{\prime}$ of the gently inverted samples and the samples containing EDTA (casein micelles denaturated) are in good agreement with the Mie calculated values (Figure 5.5), indicating a casein contribution of $0.3 \mathrm{~mm}^{-1}$ to $\mu_{s}^{\prime}$. This implies that the presumed casein concentration of $1.68 \mathrm{~mL} / \mathrm{L}$ for the Mie calculations is close to the concentration in the investigated milk samples. This finding is supported by the fact that casein concentrations in human milk show only minor biological variability compared to its fat concentration [32]. For bovine milk, the reported contribution of casein micelles to $\mu_{s}^{\prime}$ is higher [15], with approximately $1.75 \mathrm{~mm}$ ${ }^{1}$. This difference can be explained by the higher concentrations of casein in bovine milk compared to human milk $[32,36]$. Due to the small size of casein micelles compared to the fat globules in human milk, both our experimental results and Mie calculations show a negligible influence of casein on $\mu_{s}$.

The measurements in this work were performed on samples which have been frozen during storage. Although milk samples are ideally measured directly after expression from the breast, such samples were not available during this study. As a consequence, we were unable to investigate the influence of freezing, storing and thawing of milk samples on their scattering properties. Nevertheless, there are indications that freeze-thaw procedures may affect fat globule size [37]. Future research is necessary to reveal the influence of also these processing steps on the optical scattering properties of human milk.

This work shows that the scattering properties of human milk strongly depend on the preparation method, which is essential knowledge for the optical investigation of human milk, including the optical quantification of human milk 
composition. As sonication is a standard sample preparation procedure for NIR and MIR human milk analyzers, any future work on combining these methods with visible light techniques will have to take into account the effect of sonication on the optical scattering properties. As vortexing human milk samples may introduce the presence of fat globule aggregates that affect the measurement of $\mu_{s}{ }^{\prime}$, the preferred method for human milk sample preparation will be gently inverting. Another outcome of this work is the quantitative contribution of casein micelles to the scattering properties of human milk. Although casein concentrations are relatively stable for mature human milk, concentrations may be different and show more biological variability in colostrum [38]. As such, these findings are important for both the optical quantification of fat content, as well as the casein content itself.

\subsection{Conclusions}

In this study, we demonstrated experimentally and numerically that the scattering properties of human milk are strongly affected by common sample preparation methods. Sonication most strongly influenced all scattering properties of human milk due to a reduction of fat globule size, but also vortexing affected the reduced scattering coefficient $\mu_{s}^{\prime}$. For homogenization purposes, gently inverting should therefore be the preferred method. The contribution of casein micelles to the scattering properties of human milk is relatively low for $\mu_{s}$ and $g(7.1 \%$ and $1.1 \%$ at $550 \mathrm{~nm}$, respectively), but considerably larger for $\mu_{s}^{\prime}\left(29 \% \mathrm{~mm}^{-1}\right.$ at $\left.550 \mathrm{~nm}\right)$.

\section{Bibliography}

1. C. G. Victora, R. Bahl, A. J. D. Barros, G. V. A. Franca, S. Horton, J. Krasevec, S. Murch, M. J. Sankar, N. Walker, N. C. Rollins, "Breastfeeding in the 21st century: epidemiology, mechanisms, and lifelong effect," Lancet 387(10017), 475-490 (2016).

2. N. C. Rollins, N. Bhandari, N. Hajeebhoy, S. Horton, C. K. Lutter, J. C. Martines, E. G. Piwoz, L. M. Richter, C. G. Victora, and L. B. S. Grp, "Why invest, and what it will take to improve breastfeeding practices?," Lancet 387(10017), 491-504 (2016).

3. K. Hinde, and J. B. German, "Food in an evolutionary context: insights from mother's milk," J Sci Food Agr 92(11), 2219-2223 (2012). 
4. World Health Organization, "Tracking progress for breastfeeding policies and programmes: Global breastfeeding scorecard 2017," (2017) https://www.who.int/nutrition/publications/infantfeeding/global-bfscorecard-2017/en/.

5. R. Li, S. B. Fein, J. Chen, and L. M. Grummer-Strawn, "Why mothers stop breastfeeding: mothers' self-reported reasons for stopping during the first year," Pediatrics 122 Suppl 2, S69-76 (2008).

6. C. Veenstra, A. Lenferink, W. Petersen, W. Steenbergen, and N. Bosschaart, "Optical properties of human milk," Biomed Opt Express 10(8), 4059-4074 (2019).

7. N. Bosschaart, A. Leproux, O. Abdalsalam, W. P. Chen, C. E. McLaren, B. J. Tromberg, and T. D. O'Sullivan, "Diffuse optical spectroscopic imaging for the investigation of human lactation physiology: a case study on mammary involution," J Biomed Opt 24(5), 1-8 (2019).

8. K. Tanimoto, T. Kusaka, T. Nishida, K. Ogawa, I. Kato, S. Ijichi, J. Mikami, I. Sobue, K. Isobe, and S. Itoh, "Hemodynamic Changes in the Breast and Frontal Cortex of Mothers During Breastfeeding," Pediatr Res 70(4), 400-405 (2011).

9. M. van der Hoek, L. den Haan, A. Kaspers, W. Steenbergen, and N. Bosschaart, "Cutaneous perfusion of the human lactating breast: a pilot study with laser Doppler perfusion monitoring," Physiol Meas 40(5), 05NT01 (2019).

10. R. Ullah, S. Khan, S. Javaid, H. Ali, M. Bilal, and M. Saleem, "Raman spectroscopy combined with a support vector machine for differentiating between feeding male and female infants mother's milk," Biomed Opt Express 9(2), 844-851 (2018).

11. G. Fusch, N. Rochow, A. Choi, S. Fusch, S. Poeschl, A. O. Ubah, S. Y. Lee, P. Raja, and C. Fusch, "Rapid measurement of macronutrients in breast milk: How reliable are infrared milk analyzers?," Clin Nutr 34(3), 465476 (2015).

12. R. Ullah, S. Khan, A. Khan, M. Saleem, H. Ali, M. Bilal, and M. Ahmed, "Infant gender-based differentiation in concentration of milk fats using near infrared Raman spectroscopy," J Raman Spectrosc 48(3), 363 - 367 (2016). 
13. S. Arslanoglu, G. E. Moro, and E. E. Ziegler, "Adjustable fortification of human milk fed to preterm infants: does it make a difference?," J Perinatol 26(10), 614-621 (2006).

14. B. Aernouts, E. Polshin, J. Lammertyn, and W. Saeys, "Visible and nearinfrared spectroscopic analysis of raw milk for cow health monitoring: Reflectance or transmittance?," J Dairy Sci 94(11), 5315-5329 (2011).

15. S. Stocker, F. Foschum, P. Krauter, F. Bergmann, A. Hohmann, C. S. Happ, and A. Kienle, "Broadband Optical Properties of Milk," Appl Spectrosc 71(5), 951-962 (2017).

16. B. Aernouts, R. Van Beers, R. Watte, T. Huybrechts, J. Jordens, D. Vermeulen, T. Van Gerven, J. Lammertyn, and W. Saeys, "Effect of ultrasonic homogenization on the Vis/NIR bulk optical properties of milk," Colloid Surface B 126, 510-519 (2015).

17. A. Choi, G. Fusch, N. Rochow, N. Sheikh, and C. Fusch, "Establishment of micromethods for macronutrient contents analysis in breast milk," Matern Child Nutr 11(4), 761-772 (2015).

18. P. Udabage, I. R. McKinnon, and M. A. Augustin, "Mineral and casein equilibria in milk: effects of added salts and calcium-chelating agents," J Dairy Res 67(3), 361-370 (2000).

19. T. J. Farrell, M. S. Patterson, and B. Wilson, "A Diffusion-Theory Model of Spatially Resolved, Steady-State Diffuse Reflectance for the Noninvasive Determination of Tissue Optical-Properties Invivo," Med Phys 19(4), 879-888 (1992).

20. N. Bosschaart, R. Mentink, J. H. Kok, T. G. van Leeuwen, and M. C. Aalders, "Optical properties of neonatal skin measured in vivo as a function of age and skin pigmentation," J Biomed Opt 16(9), 097003 (2011).

21. N. Bosschaart, M. C. G. Aalders, T. G. van Leeuwen, and D. J. Faber, "Spectral domain detection in low-coherence spectroscopy," Biomed Opt Express 3(9), 2263-2272 (2012).

22. N. Bosschaart, D. J. Faber, T. G. van Leeuwen, and M. C. G. Aalders, "Measurements of wavelength dependent scattering and backscattering coefficients by low-coherence spectroscopy," J Biomed Opt 16(3), 030503 (2011). 
23. N. Bosschaart, M. C. Aalders, D. J. Faber, J. J. Weda, M. J. van Gemert, and T. G. van Leeuwen, "Quantitative measurements of absorption spectra in scattering media by low-coherence spectroscopy," Opt. Lett. 34(23), 3746-3748 (2009).

24. C. Veenstra, W. Petersen, I. M. Vellekoop, W. Steenbergen, and N. Bosschaart, "Spatially confined quantification of bilirubin concentrations by spectroscopic visible-light optical coherence tomography," Biomed Opt Express 9(8), 3581-3589 (2018).

25. F. C. Cheong, K. Xiao, and D. G. Grier, "Technical note: Characterizing individual milk fat globules with holographic video microscopy," J Dairy Sci 92(1), 95-99 (2009).

26. M. C. Michalski, V. Briard, and F. Michel, "Optical parameters of milk fat globules for laser light scattering measurements," Lait 81(6), 787-796 (2001).

27. A. J. Jaaskelainen, K. E. Peiponen, and J. A. Raty, "On reflectometric measurement of a refractive index of milk," J Dairy Sci 84(1), 38-43 (2001).

28. R. Attaie, and R. L. Richter, "Size distribution of fat globules in goat milk," J Dairy Sci 83(5), 940-944 (2000).

29. E. Bijl, R. de Vries, H. van Valenberg, T. Huppertz, and T. Van Hooijdonk, "Factors influencing casein micelle size in milk of individual cows: Genetic variants and glycosylation of kappa-casein," Int Dairy J 34(1), 135-141 (2014).

30. C. F. Bohren, and D. R. Huffman, Absorption and scattering of light by small particles (Wiley-Interscience, 1998).

31. J. P. Schäfer, "Implementierung und Anwendung analytischer und numerischer Verfahren zur Lösung der Maxwellgleichungen für die Untersuchung der Lichtausbreitung in biologischem Gewebe," (Universität Ulm, 2011).

32. K. Wambach, and J. Riordan, Breastfeeding and Human Lactation, Enhanced Fifth Edition (2015).

33. O. Rybak, "Milk fat in structure formation of dairy products: a review," Ukr Food J 5(3), 499-514 (2016). 
34. B. Y. Fong, C. S. Norris, and A. K. H. MacGibbon, "Protein and lipid composition of bovine milk-fat-globule membrane," Int Dairy J 17(4), 275-288 (2007).

35. K. S. Rangappa, "Studies on the refractive index of milk. 1. Observations on genuine samples," Proceedings of the National Academy of Sciences of India, Section B. Biological Sciences 25, 86-94 (1947).

36. D. Rose, "Relation between Micellar and Serum Casein in Bovine Milk," J Dairy Sci 51(12), 1897-1902 (1968).

37. K. Takahashi, K. Mizuno, and K. Itabashi, "The freeze-thaw process and long intervals after fortification denature human milk fat globules," Am J Perinatol 29(4), 283-288 (2012).

38. N. C. R. Raiha, P. A. Cooper, A. Marini, D. K. Rassin, J. Pettifor, S. Kashyap, A. Pandit, W. E. Heine, A. Fazzolari, C. Househam, and B. Lonnerdal, "Protein-Content of Human-Milk, from Colostrum to Mature Milk," Nestle Nutr Works Se 33, 87-103 (1994). 


\section{Chapter 6:}

\section{Overall discussion, future perspectives and conclusions}

Abstract: In this thesis we investigated the opportunities for spectroscopic optical coherence tomography in neonatal healthcare. We describe our vision on the (future) role of SOCT within neonatal healthcare. Further scientific research ideas, possibilities and recommendations will be given. 


\subsection{Discussion}

\subsubsection{Quantification of bilirubin and hemoglobin chromophore concentrations}

In chapters 2 and 3 , we investigated the potential of SOCT for quantification of chromophores (bilirubin and hemoglobin) relevant in neonatal healthcare. With our SOCT system, we quantified bilirubin concentrations in tissue-mimicking samples within an accuracy of $10 \%$. Furthermore, we measured total hemoglobin concentrations in human whole blood with a precision of $3.8 \mathrm{~g} / \mathrm{dL}$. All our experiments were conducted ex-vivo and in vitro in a laboratory environment. To translate our methods for quantifying bilirubin and hemoglobin into an in-vivo situation, we need to tackle multiple challenges.

As discussed in chapter 2, the precision (around $50 \mu \mathrm{M}$ ) of our sOCT system for quantifying bilirubin concentrations is similar to the precision of transcutaneous bilirubinometers. In chapter 3, we discussed the precision of our SOCT system for [tHb] measurements, which is similar to already existing non-invasive methods. Therefore, to become an alternative for invasive blood sampling, it is necessary to increase the precision of our SOCT system and associated data processing.

An important future challenge is to measure bilirubin concentrations in whole blood. The results from chapter 2 and 3 show that the bilirubin absorption is much lower than the attenuation by whole blood. For a $500 \mu \mathrm{M}$ bilirubin sample - which is the upper edge of the clinical range - we obtained $\mu_{a} \approx 4 \mathrm{~mm}^{-1}$ at the bilirubin absorption peak around $460 \mathrm{~nm}$. For human whole blood on the other hand, we already obtained an attenuation of $\mu_{t} \approx 20 \mathrm{~mm}^{-1}$ (at $460 \mathrm{~nm}$ ) for a sample containing only $6.93 \mathrm{~g} / \mathrm{dL}$ hemoglobin (associated with anemia). The relatively high attenuation of whole blood compared to bilirubin might seriously hamper the quantification of bilirubin concentrations in whole blood. An accurate estimation of the contribution of scattering and hemoglobin absorption to the total measured attenuation is critical for accurate quantification of the absorption by bilirubin. Since the precision (standard deviation) of our $\mu_{t}$ measurements on human whole blood (Figure 3.1) is in the same order of magnitude as the absorption by bilirubin in the clinical range (Figure 2.3), we argue that the accuracy and precision of our sOCT system for $\mu_{t}$ estimation should be improved (by around an order of magnitude) in order to 
successfully and accurately quantify bilirubin concentrations in whole blood. Possibilities for improvement are detailed in section 6.2.

The structure and content of the samples measured in this thesis were well known beforehand, allowing us to make a good prediction of the signal we were about to measure. This knowledge includes the location and thickness of scattering layers and location of chromophores inside the sample. In an in-vivo situation however, there is no prior knowledge on where in the skin the blood vessels suitable for our SOCT method are located. Therefore, it is required to first make a regular OCT image of the skin, from which a suitable blood vessel may be located. Hereafter, our SOCT method can be applied within that blood vessel. In chapter 3 , measurements on human whole blood were performed over a depth interval of $40 \mu \mathrm{m}$. Including the spatial resolution of $30 \mu \mathrm{m}$ at $\lambda=$ $650 \mathrm{~nm}$, the diameter of a suitable blood vessel should at least be $70 \mu \mathrm{m}$.

Since future in-vivo SOCT measurements should be locally confined to a single blood vessel, motion artifacts are an important challenge. Our method of focus tracking and zero-delay acquisition comes with superior sensitivity, but also increases the measurement time compared to conventional sOCT. Currently, the measurement time typically is in the order of minutes, depending on multiple factors including: line rate, number of averages, depth range and reference mirror oscillation frequency. Since longer measurements are more susceptible to motion artifacts, the measurement time should be reduced to a minimum. The most straightforward methods of reducing measurement time come at the cost of measurement accuracy. These are: I) reducing the acquisition time of individual frames, II) decreasing the number of averages and III) decreasing the number of depth positions for fitting our attenuation model to the SOCT data. Another possibility of decreasing the measurement time is by replacing the current piezo element - driving the oscillating reference mirror with a piezo element that supports higher frequencies. We only acquire lines within a $5 \mu \mathrm{m}$ window of the complete piezo movement after which the camera stops acquiring lines until the reference mirror again reaches this $5 \mu \mathrm{m}$ window during the next piezo sweep. Increasing the piezo frequency reduces this 'dead time' in between piezo periods. Therefore, replacing the piezo element with a higher frequency alternative will reduce measurement time, without reducing measurement accuracy. 
Measuring in-vivo requires the light to pass through a layer of skin before reaching a suitable blood vessel. Although our SOCT method can locally measure the optical attenuation without crosstalk from surrounding tissue, going through a layer of skin (i.e. scattering and absorbing layer) will still reduce the signal-to-noise ratio of the measurement. We already demonstrated the local quantification of bilirubin concentrations behind an extra scattering layer. However, these measurements were performed in tissue mimicking samples and not in human whole blood. The effect of an extra scattering layer in front of human whole blood should be subject to further research.

\subsubsection{The optical properties of human milk}

In chapter 4, we measured the full set of optical properties of human milk and their dependency on fat concentration in the visible wavelength range (450 $600 \mathrm{~nm}$ ). We hope that our work will be a starting point for further optical research onto lactation physiology and human milk. Since we showed significant correlations of $\mu_{a}, \mu_{s}, \mu_{s}{ }^{\prime}$ and $\mu_{b, N A}$ with fat concentration (up to $R_{s}=0.80$ for $\mu_{s}{ }^{\prime}$ at $550 \mathrm{~nm}$ ), visible-light optical techniques might prove to be a cost-effective alternative to their infrared counterparts [1].

In chapters 4 and 5, we showed experimentally and numerically that the scattering properties are not only affected by fat concentration, but also strongly depend on the fat globule size distribution. As a consequence, the measured scattering properties of individual milk samples show a large variation for similar fat concentrations (e.g. around a factor 2 for $\mu_{s}{ }^{\prime}$, see Figure 4.4). For comparison, biochemical methods that estimate fat concentrations have a typical coefficient of variation of only $8 \%$. Since the dependency on fat globule size distribution is different for all optical properties, it might be possible to correct for the fat globule size distribution by combining the result of several scattering properties together. This potentially leads to an even stronger correlation of the scattering properties with fat concentration. Further research is necessary to investigate the possibilities of combining the results of scattering properties.

In chapters 4 and 5, we combined SOCT and SR-DRS to estimate all of the optical properties of human milk. For this purpose, SOCT proved to be a very convenient technique. However, - as the optical properties are now known and localized measurements are of lesser importance - less complex techniques such as 
transmission and/or reflection spectroscopy might prove to be sufficient for estimating the fat and/or casein contents of human milk samples. Future research is necessary to determine the potential of other visible light techniques for analyzing the nutritional content of a human milk sample.

\subsubsection{Improvements for neonatal healthcare}

We performed research on the opportunities of biomedical optics for neonatal healthcare. In this work, we mainly focused on showing the potential of optical techniques such as SOCT and SR-DRS for improving neonatal healthcare. However, more research is required to bring our methods into the clinic and/or home care situation.

It is necessary to explore the possibilities of SOCT for non-invasive in-vivo measurements of bilirubin and hemoglobin concentrations in neonates and to compare these results with existing non-invasive techniques [2, 3]. Potential advantages of SOCT are a potential increase in accuracy compared to conventional spectroscopic methods, due to quantitative measurements within a single blood vessel and the possibility of measuring local chromophore extravasation in the skin. The latter is possible since the optical path length can be controlled in SOCT, allowing for individual local measurements inside and outside of a blood vessel.

Although we investigated the correlation between the optical properties of milk and its fat concentration, the clinically relevant parameter is the accuracy with which visible-light optical techniques are able to estimate the nutritional content of human milk. Furthermore, it is very favorable to develop a relatively small and low-cost device, such that the technology can become accessible on a larger scale.

Another potential application of SOCT is measuring local differences in chromophore concentrations. For neonatal healthcare, one of these applications might be estimating the extravasation of bilirubin from blood vessels into surrounding tissue, by quantifying the bilirubin concentrations within a single blood vessel and within tissue adjacent to this blood vessel. 


\subsection{Future perspectives}

\subsubsection{Estimating blood flow}

Another capability of OCT is localized measurements of blood flow speeds [4]. Estimating the flow speed within individual blood vessels is interesting for our purposes as the low shear rate near the vessel wall gives rise to a red blood cell free layer, while red blood cells accumulate in the central zone of the capillary [5]. As a result, the flow within the blood vessels causes inhomogeneities in the distribution of optical absorption and scattering throughout the vessel. Therefore, it is interesting to investigate whether it is possible to (partly) correct for these inhomogeneities by measuring the flow speeds throughout the vessel.

\subsubsection{Other chromophores}

In this work, we mainly looked into the potential of visible-light SOCT and SRDRS for measuring bilirubin, hemoglobin and fat concentrations. In principle, our SOCT method for quantifying chromophore concentrations works for all chromophores, as long as they exhibit a known absorption spectrum within the same wavelength range as the SOCT system is operating in. Therefore, extending the SOCT system into the infrared wavelength range may allow for local absorption-based quantification of water [6] and fat [7] concentrations.

\subsubsection{Into the clinic; Handheld measurements}

Bringing SOCT measurements into the clinic requires the development of a fiber based handheld probe, which can be placed onto the newborn's skin. Such a probe should include a scanning mirror, required to enable 2 and/or 3 dimensional imaging to locate suitable blood vessels. Furthermore, the probe has to include a translatable focus lens, necessary for focus tracking during the sOCT measurement.

Currently, we are developing such a probe in cooperation with VDL ETG (Almelo, The Netherlands). A first prototype is available and is currently being integrated into our SOCT setup. Remaining challenges include efficient single mode fiber coupling, synchronization of the scanning mirror with the camera of the SOCT setup and validation measurements.

Future research is required to uncover whether a system with such a probe is able to perform measurements with a similar signal-to-noise ratio as the open- 
air system. The probe's capabilities for identifying suitable blood vessels and steering the beam towards such blood vessels should be investigated. Furthermore, the effect of optical attenuation by a scattering layer (e.g. skin) in front of blood vessels should be studied. Since a handheld probe will be prone to movement, the effects of movement artefacts should be researched. Finally, the complete setup should be converted into a mobile setup which is electrically and optically safe and mechanically stable.

\subsection{Conclusions}

Throughout this thesis, we investigated multiple potential applications of SOCT in the field of neonatal healthcare. Our results indicate that SOCT might play an important future role for the non-invasive quantification of chromophore concentrations in blood, of which the most promising results were obtained for hemoglobin.

We showed that SOCT - since this technique allows for controlling the optical path length through tissue - is able to locally quantify the attenuation for $\mu_{t}$ values up to at least $60 \mathrm{~mm}^{-1}$. As a result, our SOCT method succeeded in quantitatively estimating bilirubin (within $10 \%$ accuracy, chapter 2) and hemoglobin concentrations (with $3.8 \mathrm{~g} / \mathrm{dL}$ precision, chapter 3 ). Furthermore, we successfully mapped the full set of optical properties of human milk and showed that $\mu_{a}, \mu_{s}, \mu_{s}{ }^{\prime}$ and $\mu_{b, N A}$ significantly correlate with fat concentration (chapter 4). Finally, we showed that the scattering properties of human milk strongly depend on the preparation method used to homogenize the sample (chapter 5).

\section{Bibliography}

1. G. Fusch, N. Rochow, A. Choi, S. Fusch, S. Poeschl, A. O. Ubah, S. Y. Lee, P. Raja, and C. Fusch, "Rapid measurement of macronutrients in breast milk: How reliable are infrared milk analyzers?," Clin Nutr 34(3), 465476 (2015).

2. N. Bosschaart, J. H. Kok, A. M. Newsum, D. M. Ouweneel, R. Mentink, T. G. van Leeuwen, and M. C. G. Aalders, "Limitations and Opportunities of Transcutaneous Bilirubin Measurements," Pediatrics 129(4), 689-694 (2012).

3. M. Y. Shamir, A. Avramovich, and T. Smaka, "The Current Status of Continuous Noninvasive Measurement of Total, Carboxy, and Methemoglobin Concentration," Anesth Analg 114(5), 972-978 (2012). 
4. N. Weiss, T. G. van Leeuwen, and J. Kalkman, "Localized measurement of longitudinal and transverse flow velocities in colloidal suspensions using optical coherence tomography," Phys Rev E 88(4), (2013).

5. O. K. Baskurt, M. R. Hardeman, and M. W. Rampling, "Handbook of Hemorheology and Hemodynamics," (2007).

6. G. M. Hale and M. R. Querry, "Optical-Constants of Water in 200-nm to 200- $\mu \mathrm{m}$ Wavelength Region," Appl Optics 12(3), 555-563 (1973).

7. S. Prahl, "Optical Absorption of Fat", retrieved 18-01-2019. 


\section{List of symbols}

Angular wave number $k$

Anisotropy $G$

Absorption coefficient $\quad \mu_{a}$

Absorption efficiency $\quad Q_{a}$

Attenuation coefficient $\quad \mu_{t}$

Axial resolution $\Delta d$

Backscattered intensity at zero-delay $S$

Backscattering coefficient $\mu_{b}$

Backscattering coefficient (within NA) $\quad \mu_{b, N A}$

Center wavelength

Coefficient of determination

Coherence length

Concentration (particles per volume) N

Concentration (volume fraction) $C$

Deoxygenated hemoglobin concentration $[\mathrm{Hb}]$

Depth $d$

Depth relative to maximum depth $\tau$

Detected intensity $\quad I_{\text {det }}$

Diameter $D$

Diffuse reflectance $R$

Doppler frequency shift $\quad f_{d}$

Effective absorption coefficient $\quad \mu_{a \text {,effective }}$

Filtered intensity $\quad l_{\text {filt }}$

Focus position $\quad l_{f}$

Free absorption coefficient $\quad \mu_{a, f r e e}$

Intensity (after applying STFT) ISTFT

Maximum detectable depth $\quad d_{\max }$

Optical path length difference $\quad \triangle O P L$

Oxygenated hemoglobin concentration $\quad\left[\mathrm{HbO}_{2}\right]$

Rayleigh length $\quad Z_{R}$

Reduced scattering coefficient $\quad \mu_{s}{ }^{\prime}$

Reference arm length $\quad x_{r}$

Reference arm intensity $I_{r}$

Reference arm step size $d x$ 
Refractive index

$n$

Sample arm length

Sample arm intensity

$x_{s}$

Scaling factor

Scatter power

a (for sOCT), 8 (for SR-DRS)

Scattering coefficient

$b$

Scattering cross section

$\mu_{s}$

Scattering efficiency

Scattering phase function

$\sigma_{s}$

$Q_{s}$

$P$

Source detector distance

Spearman correlation coefficient

Spectral bandwidth

Spectral resolution (of spectrometer)

$r$

$R_{s}$

$\lambda_{F W H M}$

Spectral STFT window size

$\delta \lambda$

System efficiency term

Time

$\Delta \lambda$

Total hemoglobin concentration

Velocity of the reference mirror

$\zeta$

$t$

[tHb]

Wavelength

$v_{r}$

Wavelength spacing between pixels

$\lambda$

$\xi \lambda$

Zero-delay position relative to sample's

$d_{z D}$

surface 


\section{List of abbreviations}

Diffuse optical imaging DOI

Ethylenediaminetetraacetic EDTA

Laser Doppler perfusion monitoring LDPM

Low coherence spectroscopy LCS

Mid-infrared MIR

Near-infrared NIR

Near-infrared spectroscopy NIRS

Numerical aperture NA

Optical coherence tomography $\quad$ OCT

Optical path length $\quad$ OPL

Perception of insufficient milk supply PIM

Phosphate buffered saline PBS

Photoacoustic mammography PAM

Point spread function PSF

Red blood cell $\quad$ RBC

Short time Fourier transformation STFT

Slgnal-to-noise ratio SNR

Spatially resolved diffuse reflectance spectroscopy SR-DRS

Spectroscopic optical coherence tomography SOCT

Standard deviation SD

Visible light spectroscopic optical coherence tomography Vis-sOCT

Zero-delay

ZD 


\section{Summary}

In this thesis, we explored the opportunities of spatially resolved optical spectroscopy for neonatal healthcare. Hereto, we developed a setup that makes use of spectroscopic optical coherence tomography (sOCT). This technique allows for spectroscopic measurement of the attenuation of light within a confined measurement volume, without being influenced by surrounding tissues. Throughout this thesis, we explored the possibilities of SOCT for estimating tissue properties which are relevant to newborns. In this way, we hope to contribute to neonatal healthcare by enabling a non-invasive alternative for invasive blood sampling and by enabling more scientific research into human milk and lactation.

The measured attenuation of light as a function of depth in conventional SOCT is not only affected by the optical properties of the sample, but also by influences of the system itself. Developing an SOCT system that implements both zero-delay acquisition and focus tracking, allowed us to measure the attenuation of light as a function of depth without being affected by the properties of the system. Another important advantage of combining zero-delay acquisition with focus tracking is measuring with optimal sensitivity at every depth inside the sample. The attenuation of light is directly related to the concentration of chromophores and scattering particles. Examples of chromophores are hemoglobin and bilirubin. An example of scattering particles are fat globules in milk, which also cause the white appearance of milk. By using sOCT for local measurements (for example within a single blood vessel) of the attenuation of light, it is possible to non-invasively estimate the concentration of chromophores and scattering particles.

\section{Chapter 2}

In chapter 2 we explored the possibilities of our SOCT system for estimating bilirubin concentrations. Bilirubin is a toxic, yellow breakdown product that - at elevated levels - causes jaundice. If bilirubin levels are highly elevated for a longer time, this might cause irreversible brain damage. Newborns are at high risk for elevated bilirubin levels, since their liver is usually not yet capable of processing all bilirubin. Therefore, it is very important to closely monitor the bilirubin concentrations of newborns. Currently, bilirubin concentrations are measured by drawing blood through an invasive heel stick, followed by 
laboratory analysis. This heel stick induces pain and stress. Furthermore, drawing blood from newborns is very undesirable due to the small blood volume of newborns. We show in tissue mimicking samples that our SOCT system is capable of estimating bilirubin concentrations throughout the entire clinical range within an accuracy of $10 \%$.

\section{Chapter 3}

Hemoglobin is the protein in blood responsible for transporting oxygen to the organs. Hemoglobin is what gives the blood its red color. Reduced concentrations of hemoglobin induce anemia. In chapter 3, we investigated whether our SOCT system is able to measure hemoglobin concentrations in human blood. Hereto, we obtained donor blood and estimated the hemoglobin concentrations ex-vivo with both our SOCT system and a commercially available reference method. The results show that our SOCT system can measure hemoglobin concentrations in human blood with a precision of $3.8 \mathrm{~g} / \mathrm{dL}$.

\section{Chapter 4}

In chapter 4 we mapped the optical properties of human milk in the visible wavelength range $(450-600 \mathrm{~nm})$ with both experimental and numerical techniques. We show a significant correlation between the chemically derived fat concentration and multiple optical properties. We hope that this study serves as a starting point for further research into the possibilities of visible light methods for estimating the nutritional content of milk. These visible light methods might prove to be cost-effective alternative for existing milk analysis techniques based on infrared light. Furthermore, we believe that knowledge of the optical properties of human milk enables for more extensive optical research in the field of human lactation.

\section{Chapter 5}

It is important to homogenize milk samples prior to analyzing them. Multiple techniques are available for homogenizing milk samples. Infrared-based optical techniques, for example, require sonication of the milk samples before their nutritional content can be estimated. Sonication means that the sample is homogenized by ultrasonic waves, a technique which also reduces the diameter of the fat globules in the milk. Therefore, in chapter 5 we investigate how the scattering properties of the milk are affected by commonly used 
homogenization procedures. The results show a high dependency of the scattering properties on the used homogenization method.

\section{To finalize}

In this thesis, we explored the opportunities and possibilities of biomedical optics for neonatal healthcare. Although we obtain promising results, all experiments were conducted in a laboratory setting. Future research has to increase the precision for quantifying hemoglobin concentrations and show the potential of the techniques used throughout this thesis in a clinical setting.

We showed that SOCT is sensitive to both absorption and scattering of light and is capable of estimating these quantities without crosstalk from surrounding tissue. Although clinical implementation requires further improvement in terms of measurement precision, we expect that SOCT may play an important future role in the non-invasive estimation of chromophore concentrations in single blood vessels. 


\section{Nederlandstalige samenvatting}

In dit proefschrift hebben we de mogelijkheden en kansen van ruimtelijk opgeloste spectroscopie in de neonatale zorg onderzocht. Hiertoe hebben we een opstelling ontwikkeld die gebruik maakt van spectroscopische optische coherentie tomografie (sOCT). Met deze techniek is het mogelijk om per golflengte de verzwakking van licht als functie van meetdiepte te meten binnen een beperkt meetvolume, zonder dat deze meting beïnvloed wordt door omliggend weefsel. Door dit hele proefschrift heen hebben we de mogelijkheden van SOCT onderzocht voor het bepalen van weefseleigenschappen die zeer relevant zijn voor pasgeborenen, om op deze manier een bijdrage te leveren aan de neonatale zorg.

Bij conventionele sOCT wordt de gemeten verzwakking van licht met diepte niet alleen bepaald door de optische eigenschappen van het monster, maar ook door eigenschappen van het systeem zelf. Door een SOCT systeem te ontwikkelen dat tijdens een meting zowel het 'nulpunt' als het focus van het licht mee verplaatst met de meting, kunnen wij met ons systeem de verzwakking van licht in een monster meten zonder dat de eigenschappen van het systeem daarin een rol spelen. Een belangrijk bijkomend voordeel is dat ons systeem op elke diepte in het monster met optimale gevoeligheid meet. De verzwakking van licht houdt direct verband met de concentratie van chromoforen en verstrooiende deeltjes binnen het meetvolume. Voorbeelden van moleculen die chromoforen bevatten zijn hemoglobine en bilirubine. Een voorbeeld van verstrooiende deeltjes zijn vetglobulen in melk, welke ervoor zorgen dat melk wit is. Door met SOCT lokaal (bijvoorbeeld binnen een enkel bloedvat) de verzwakking van licht te meten, is het mogelijk om lokaal op nietinvasieve wijze de concentraties van chromoforen en verstrooiende deeltjes te bepalen.

\section{Hoofdstuk 2}

In hoofdstuk 2 hebben we de mogelijkheden onderzocht voor het gebruik van ons SOCT systeem voor het meten van bilirubine concentraties. Bilirubine is een giftige, gele afvalstof die bij te hoge concentraties geelzucht veroorzaakt. Wanneer bilirubineconcentraties langdurig erg verhoogd zijn, kan dit leiden tot onherstelbare hersenschade. Pasgeborenen zijn extra gevoelig voor verhoogde bilirubineconcentraties, aangezien hun lever meestal nog niet goed in staat is 
om alle bilirubine op een goede manier af te voeren. Het is daarom belangrijk om de bilirubine concentraties bij pasgeborenen goed in de gaten te houden. Dit wordt momenteel gedaan door middel van een hielprik, waarbij bloed wordt afgenomen en wordt getest in een laboratorium. Deze hielprik gaat gepaard met pijn en stres. Verder is een bloedafname bij pasgeborenen ook ongewenst gezien het kleine bloedvolume. We laten zien in monsters die weefsel nabootsen dat ons SOCT systeem in staat is om bilirubineconcentraties te bepalen over het gehele klinische gebied, met een nauwkeurigheid binnen 10 $\%$.

\section{Hoofdstuk 3}

Hemoglobine is het eiwit in het bloed dat verantwoordelijk is voor het vervoer van zuurstof naar de organen en geeft bloed zijn rode kleur. Een verlaagde concentratie hemoglobine veroorzaakt bloedarmoede. In hoofdstuk 3 hebben we gekeken of ons SOCT systeem ingezet kan worden om hemoglobineconcentraties te meten in menselijk bloed. Hiertoe hebben we donorbloed gebruikt en hiervan zowel de concentratie hemoglobine bepaald met een referentiemethode en ex-vivo met ons SOCT systeem. Hieruit blijkt dat ons SOCT systeem in staat is om hemoglobineconcentraties in menselijk bloed te bepalen met een precisie van $3.8 \mathrm{~g} / \mathrm{dL}$.

\section{Hoofdstuk 4}

In hoofdstuk 4 hebben we de optische eigenschappen in het zichtbare gebied $(450-600 \mathrm{~nm})$ van menselijke moedermelk in kaart gebracht, zowel experimenteel als numeriek. Door de optische eigenschappen van melkmonsters uit te zetten tegen de vetconcentratie, laten we zien dat meerdere optische eigenschappen een significante correlatie vertonen met de vetconcentratie van de melk. Met deze studie hopen we een voorzet te geven naar meer onderzoek richting de mogelijkheden van technologieën op basis van zichtbaar licht voor het bepalen van de voedingswaarden van melk. Technologieën op basis van zichtbaar licht zijn mogelijk een goedkoper alternatief voor de reeds bestaande infrarood melk-analyse technieken.

Hoofdstuk 5

Voordat melkmonsters geanalyseerd kunnen worden, is het belangrijk om de monsters goed te homogeniseren. Er zijn meerdere technieken om 
melkmonsters te homogeniseren, zo vereisen veel infrarood meetmethodes dat de monsters eerst gesonificeerd worden. Sonificeren betekent dat het monster door middel van ultrageluid wordt gehomogeniseerd, waarbij ook de diameter van de vetglobulen vermindert. In hoofdstuk 5 onderzoeken we hierom de invloed van verschillende veel voorkomende homogenisatie technieken op de verstrooiende eigenschappen van moedermelk. We laten zien dat de verstrooiende eigenschappen sterk afhankelijk zijn van de grootte van de vetglobulen en dus van de gebruikte homogenisatiemethode.

\section{Ter afsluiting}

In dit proefschrift hebben we meerdere toepassingen van SOCT laten zien die veelbelovend voor de neonatale zorg zijn. Ondanks deze veelbelovende resultaten moet er benoemd worden dat al onze experimenten in het laboratorium zijn uitgevoerd. Toekomstig onderzoek zal de potentie van de in dit proefschrift geïntroduceerde technieken in de kliniek moeten uitwijzen.

We hebben laten zien dat SOCT gevoelig is voor zowel absorptie als verstrooiing van licht en deze grootheden lokaal kan kwantificeren zonder invloed van omliggend weefsel. Ondanks dat een klinische implementatie verdere verbeteringen vergt in de meetprecisie, verwachten we dat SOCT in de toekomst een belangrijke rol kan spelen voor het niet-invasief bepalen van chromofoorconcentraties binnen een enkel bloedvat. 


\section{List of publications and presentations}

\section{Peer-reviewed articles related to this thesis}

- Colin Veenstra, Wilma Petersen, Ivo M. Vellekoop, Wiendelt Steenbergen, and Nienke Bosschaart, "Spatially confined quantification of bilirubin concentrations by spectroscopic visible-light optical coherence tomography," Biomed. Opt. Express 9(8), 3581-3589 (2018)

- Colin Veenstra, Anki Lenferink, Wilma Petersen, Wiendelt Steenbergen, and Nienke Bosschaart, "Optical properties of human milk," Biomed. Opt. Express 10(8), 4059-4074 (2019)

- Colin Veenstra, Saskia Kruitwagen, Dafne Groener, Wilma Petersen, Wiendelt Steenbergen and Nienke Bosschaart, "Quantification of total haemoglobin concentrations in human whole blood by spectroscopic visible-light optical coherence tomography," Sci. Rep. 9, 15115 (2019)

- Colin Veenstra, Dayna E. Every, Wilma Petersen, Johannes B. van Goudoever, Wiendelt Steenbergen, and Nienke Bosschaart, "The dependency of the optical scattering properties of human milk on casein content and common sample preparation methods," J. Biomed. Opt. 25(4), 045001 (2020)

\section{Other peer-reviewed articles}

- Yolanda Villanueva, Colin Veenstra, and Wiendelt Steenbergen, "Measuring absorption coefficient of scattering liquids using a tube inside an integrating sphere," Appl. Opt. 55(11), 3030-3038 (2016) 


\section{Oral presentations}

- Colin Veenstra, Lot Jeurink, Ivo M. Vellekoop, and Nienke Bosschaart, "Needless Needles," MIRA Day, Enschede, The Netherlands (2016).

- Colin Veenstra, Saskia Kruitwagen, Dafne Groener, Wilma Petersen, Wiendelt Steenbergen, and Nienke Bosschaart, "Quantification of hemoglobin concentrations in whole blood by visible-light spectroscopic optical coherence tomography," SPIE Photonics West, San Francisco, United States of America (2019).

\section{Poster presentations}

- Colin Veenstra, Lot Jeurink, and Nienke Bosschaart, “Non-invasive blood analysis for neonates, measuring bilirubin and hemoglobin levels using Low Coherence Spectroscopy," Photonics NL, Veldhoven, The Netherlands (2016).

- Colin Veenstra, Lot Jeurink, Ivo M. Vellekoop, and Nienke Bosschaart, "Bilirubin and hemoglobin absorption quantification by visible light spectroscopic OCT," SPIE Photonics West, San Francisco, United States of America (2017).

- Colin Veenstra, Lot Jeurink, Saskia Yperlaan, Ivo M. Vellekoop, and Nienke Bosschaart, "Bilirubin and hemoglobin absorption quantification by visible light spectroscopic OCT," International Graduate summer school Biophotonics 17, Sankt Ibb, Sweden (2017).

- Colin Veenstra, Lot Jeurink, Ivo M. Vellekoop, Wilma Petersen, and Nienke Bosschaart, "Quantification of Bilirubin concentration by spectroscopic optical coherence tomography," SPIE Photonics West, San Francisco, United States of America (2018).

- Colin Veenstra, Anki Lenferink, Dayna Every, Wilma Petersen, Wiendelt Steenbergen, and Nienke Bosschaart, "The optical properties of human milk," SPIE Photonics West, San Francisco, United States of America (2019). 


\section{Dankwoord / Acknowledgements}

Allereerst wil ik graag mijn begeleider en co-promotor Nienke Bosschaart bedanken dat ik 4 jaar lang heb mogen werken aan dit mooie project. Door deze mogelijkheid heb ik veel mogen leren over optica in het algemeen en optische coherentie tomografie in het bijzonder. Bedankt voor de vele hulp en goede discussies de afgelopen 4 jaar :-)

Hiernaast wil ik Ivo Vellekoop graag bedanken voor mijn tijdelijke begeleiding. Bedankt voor de tijd die je in mij hebt gestoken en voor je goede hulp tijdens het uitwerken van de sOCT data verwerking.

Ik wil graag mijn promotor, Wiendelt Steenbergen bedanken voor de tijd en energie die in mij is gestoken en voor de mogelijkheid om mijn promotieonderzoek te mogen doen bij de BMPI onderzoeksgroep.

Ik wil de leden van de promotiecommissie, prof.dr. S. Manohar, prof.dr. A.G.J.M. van Leeuwen, dr.ir. D.J. Faber en prof.dr. A. Amelink bedanken voor de energie en tijd die ze hebben gestopt in het lezen en beoordelen van dit proefschrift.

Wilma Petersen, bedankt voor je vele hulp in het chemische lab. Ik zal de gezelligheid tijdens onze duizenden pogingen om goede bilirubine monsters te maken niet snel vergeten. Johan van Hespen, veel dank voor alle technische hulp die ik de afgelopen 4 jaar heb gekregen en voor alle tijd die je genomen hebt om mij te helpen tijdens het ontwikkelen van de Labview toepassingen. Syl, bedankt voor het altijd goed regelen van eigenlijk alles. En natuurlijk voor de gezellige gesprekken, ook al waren die over voetbal de laatste jaren niet zo heel positief ;).

Ik wil graag Lot, Jeffrey, David, Saskia, Jelle, Dafne en Saskia bedanken voor hun rol in het project tijdens hun afstudeeropdrachten. Dankzij jullie inzet hebben we mooie stappen kunnen maken. Het was erg fijn en gezellig om met jullie samen te kunnen werken :).

Furthermore I want to thank my office mates throughout the years. Altaf, thank you for all your help and for being a friend inside and outside (especially at 
Highway 395) the office. Mithun, thank you for our interesting and mostly funny conversations. Ata, bedankt voor de Iraanse snacks en dat je ons hebt geleerd hoe we met ons bureau om zouden moeten gaan. $Y$, bedankt voor de vele, interessante en unieke gesprekken, mooie roadtrip en natuurlijk de echte Vietnamese loempia's. Damien, merci for sharing a good taste of music. Dayna, bedankt voor de vele gezamenlijke discussies over moedermelk en de gezelligheid. Carlos, thank you for our discussions about the OCT system and learning me a lot about polarization sensitive OCT.

Wieders wol'k nog geern David en Yoeri bedàànkn veur a'k doar aajt ' $n$-en lök in mien moodersproake van mie af kon kuiern, nööln en dramm'n. En hiernaast natuurlijk Gerwin, Sjoukje en Maura die dit altijd maar hebben moeten verdragen tijdens onze vele koffiepauzes en natuurlijk de roadtrips in Zweden en de VS. Ondanks dat het misschien vaak niet zo over kwam, was het allemaal 'wel oké' ;-).

Verder wil ik graag in een aparte alinea Sjoukje bedanken voor het aanpassen en converteren van de figuren in hoofdstuk 5 .

Dan is er nog de bieralinea. Ik wil graag Bart bedanken voor zijn goede informatie over allerlei biersoorten en hiernaast ook altijd je goede raad in het algemeen. Onno bedankt voor dat je me hebt laten zien hoe bier wordt gebrouwen en natuurlijk ook voor alle raadseltjes. And of course Tzu-Lun, thanks for sharing your favorite beers with me.

Mijn grote dank gaat ook uit naar 'citytrippers' BW, Dow, Eek, Beer en JJH. Ondanks dat we allemaal ambitieuze mensen zijn die streven verschillende stappen te zetten op de professionele ladder met tegenwoordig een druk leven, zien wij toch nog met enige regelmaat een mogelijkheid te vinden om een culturele trip aan te gaan. Ik ben van mening dat deze 'tripjes' mij kennis hebben laten maken met vele andere gebruiken en tradities die mij hebben geholpen om een beter mens te worden en mezelf te ontplooien tot degene die ik nu ben.

Oawer De Chillbazen - Bas, Brian, Daniel, Jarno, Jesse, Mart, Sjoerdpls - kan'k elk geels niks goods bedèènkn, ieleu hebt mie de letste 4 joar nens met hölpn. $\bigvee ø r a l$ Mart nich, aajt met die oale pussy-rock gangs te wean.

Dan de 'Kamereu' - Davy, C(h)ristian, Daan, FJ, Gijs, Jelln, Ronald, Rick, Ylan en JW- Ondanks dat we elkaar niet meer dagelijks zien, ben ik blij dat jullie soms 
nog de moeite nemen om tegen mij aan te kijken. Ook al lijkt dat meestal meer een excuus te zijn om bier te drinken. Maar goed, beter gemaakte vrienden dan geen vrienden.

Dan wol'k nog geern pa bedàànkn veur 't mangs vervang'n van'n inktpatroon'n van'n printer, den overigens niks van doon hef had met dit boek.

$M a$, ie wol'n a'k oe de èllèènd nich an zol doen om hier wat oawer oe toe te zett'n, dus dat doo'w lekker toch. Bedankt!

Marieke bedankt voor het aanhoren van al mijn gezeur, knap dat je er nog steeds bent.

Kalm an en goadgoan,

Colin 


\section{About the author}

Colin Veenstra was born at 27 September 1991 in Almelo, Netherlands. He performed his studies at the University of Twente, where he obtained his bachelor's degree in Biomedical Technology in 2013 and his master's degree in Biomedical Engineering in 2015. During his master's research he investigated the use of integrating spheres for measuring the optical absorption by scattering liquid samples.

He worked at his PhD-research from 2015 until 2019 at the Biomedical Photonic Imaging group at the University of Twente. In this research, he investigated the potential of spectroscopic optical coherence tomography for multiple applications in the neonatal healthcare. These application include measuring the optical properties of human milk and the quantification of bilirubin and hemoglobin concentrations. 